\title{
Convergência de Conjuntos de Nível de Polinômios Homogêneos
}

\author{
Guilherme R. A. Molina Benites \\ DisserTAÇÃo APRESENTADA \\ $\mathrm{AO}$ \\ Instituto de Matemática E Estatística \\ DA \\ Universidade de São Paulo \\ PARA \\ OBTENÇÃO DO GRAU \\ DE \\ Mestre em Matemática
}

Área de Concentração: Análise

Orientador: Profa. Dra. Mary Lilian Lourenço

-São Paulo, junho de 2006- 


\section{Convergência de Conjuntos de Nivel de Polinômios Homogêneos}

Este exemplar corresponde à redação final da dissertação de mestrado devidamente corrigida e defendida por Guilherme R. A. M. Benites e aprovada pela comissão julgadora.

São Paulo, 23 de junho de 2006.

Banca examinadora:

- Profa. Dra. Mary Lilian Lourenço --.- IME-USP

- Profa. Dra. Maria Sueli Marconi Roversi - IMECC-UNICAMP

- Prof. Dr. Leonardo Pellegrini Rodrigues -- USP Leste 
À Paula 


\section{Agradecimentos}

Devo o meu primeiro agradecimento ao Prof. Benê, que me ensinou Matemática ao longo do Ensino Médio. Seu carisma e sua dedicação foram fundamentais para a escolha que fiz ao concluir a primeira etapa da minha vida acadêmica.

Agradeço também aos meus familiares, mas principalmente a meus pais, Ilka e Luis, que sempre me incentivaram e apoiaram, independentemente das escolhas que fiz. É impossível expressar em palavras o que significam para mim.

Agradeço aos colegas de graduação, sobretudo à turma do "Básico 2000". É uma imensa satisfação saber que os tenho por perto, ainda que tenhamos seguido rumos distintos. Em especial, pela ajuda e pela amizade, gostaria de agradecer a Bruno Leite e a Sérgio Malacrida. Ao primeiro, ainda devo minha gratidão pela ajuda nos momentos finais da conclusão desse trabalho.

Também não posso deixar de agradecer a dois colegas que colaboraram imensamente durante o mestrado: Vinicius Cifú Lopes, companheiro em diversas disciplinas, e Neusa Nogas, que pacientemente assistiu à maioria de meus seminários.

Agradeço, ainda, aos colegas de Risk Office, em especial a Fábio Perdiz, Fernando Lovisotto, Leonardo Bortoloto, Roberto Masaishi Yoshikawa e Wanderlei Lima.

À Paula Stefanoni, por ter sido responsável por tornar tudo isso mais fácil e agradável.

À banca examinadora, pelas sugestões apresentadas para a versão final. Em especial, ao Prof. Dr. Leonardo Pellegrini, pelos aprimoramentos sugeridos para algumas demonstrações apresentadas ao longo da dissertação.

E, finalmente, à minha orientadora, Prof. Dra. Mary Lilian Lourenço. Não posso agradecer-lhe somente pela orientação. Tudo o que aprendi trabalhando com ela, tanto pessoal como profissionalmente, é a maior lição que fica desse período. Ficaria feliz em poder, algum dia, ensinar a alguém tudo o que ela me ensinou. 
"Talvez o mundo não seja pequeno Nem seja a vida um fato consumado Quero inventar o meu próprio pecado Quero morrer do meu próprio veneno."

(Chico Buarque e Gilberto Gil, em "Cálice" - 1973) 


\title{
Resumo
}

O objetivo principal desse trabalho é estudar algumas caracterizações das convergências pontual e uniforme de sequiências de polinômios homogêneos em espaços de Banach através de alguns tipos de convergência topológica de seus conjuntos de nível.

Para isso, definimos alguns tipos de convergencia de conjuntos e analisamos suas principais propriedades. Inicialmente, encontramos caracterizações de convergências de funcionais lineares contínuos, que são generalizadas para o contexto de polinômios homogêneos, tanto no caso real como no caso complexo. Esse trabalho é baseado no texto científico de J. Ferrera, publicado em 1998 no periódico Transactions of the American Mathematical Society, vol.350.

\begin{abstract}
The main purpose of this work is to study characterization of pointwise and uniform convergence of sequences of homogeneous polynomials on a Banach space by means of some kinds of topological convergence of their level sets.

We define some kinds of convergence of sets and analyze their main properties. We first study some characterization of convergence of sequences of continous linear functionals and then generalize them to the context of homogeneous polynomials, both in the real and the complex cases. The main results presented here were obtained by J. Ferrera, and published at Transactions of the American Mathematical Society, vol.350.
\end{abstract}




\section{Conteúdo}

$\begin{array}{ll}\text { Introdução } & \mathbf{3}\end{array}$

$\begin{array}{ll}\text { Notações } & 4\end{array}$

1 Preliminares $\quad 5$

1.1 Estruturas em Espaços Vetoriais . . . . . . . . . . . . . . . . . . 5

1.2 Teoremas Fundamentais e Espaços Reflexivos . . . . . . . . . . . . . . . 7

1.3 Topologias Fraca e Fraca- $\ldots \ldots \ldots \ldots \ldots \ldots$

2 Convergência de Conjuntos $\quad 14$

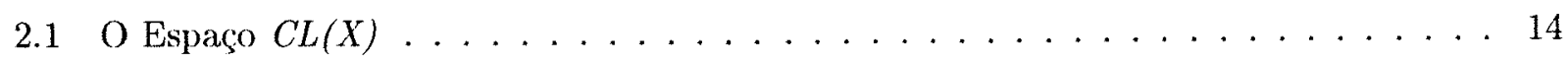

2.2 A distância de Hausdorff $\ldots \ldots \ldots \ldots \ldots \ldots \ldots \ldots \ldots$

2.3 A Convergência de Wijsman . . . . . . . . . . . . . . . . . 17

2.4 A convergência de Kuratowski . . . . . . . . . . . . . . . . . . . . 19

2.4 .1 A topologia de Fell . . . . . . . . . . . . . . . . . . 22

2.4 .2 Espaços com nice closed balls . . . . . . . . . . . . . . . 24

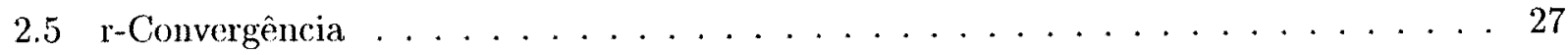

2.6 A Convergência de Mosco . . . . . . . . . . . . . . . 30

3 Convergência de conjuntos de nível de funcionais lineares contínuos $\quad 37$

3.1 Convergência Fraca- $\ldots \ldots \ldots \ldots \ldots \ldots \ldots$

3.2 Convergência em Norma . . . . . . . . . . . . . . . . 43

4 Polinômios Homogêneos

4.1 Aplicações Multilineares . . . . . . . . . . . . . . . . . . . . . 52

4.2 Aplicações Multilineares Simétricas . . . . . . . . . . . . . . . . 58

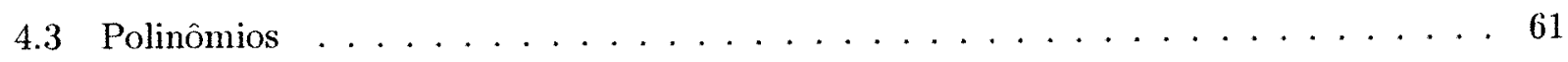

4.4 Diferenciabilidade . . . . . . . . . . . . . . . . 65 
5 Convergência de conjuntos de nível de polinômios

5.1 Introdução . . . . . . . . . . . . . . . . . 68

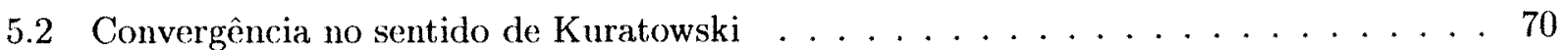

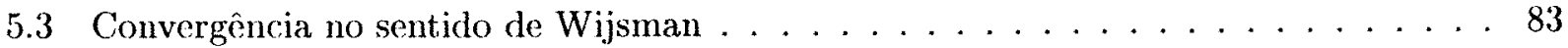

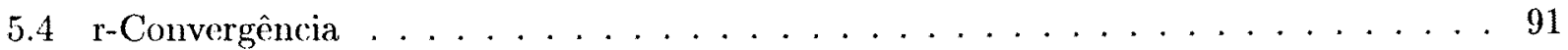

$\begin{array}{ll}\text { Bibliografia } & 98\end{array}$ 


\section{Introdução}

Esse trabalho apresenta algumas caracterizaçòes de convergências, tanto de funcionais lineares contínuos como de polinômios homogêneos, a partir de alguns tipos de convergência de seus conjuntos de nível.

As relações apresentadas no caso de funcionais lineares contínuos foram obtidas por G. Beer, em [3], enquanto que J. Ferrera, em [8], apresentou uma generalização destes resultados no contex to de polinômios homogêneos em espaços de Banach. O estudo desses textos exige uma série de pré-requisitos, que são apresentados da forma como se segue.

No Capítulo 1, estudamos espaços normados, espaços de Banach e algumas convergências que ocorrem nesses espaços: convergência em norma, convergência fraca e convergência fraca- . Também se apresentam alguns resultados sobre espaços reflexivos.

o Capítulo 2 dedica-se ao estudo de alguns tipos de convergências de conjuntos, que serão utilizadas nas caracterizações a que visamos encontrar.

No Capítulo 3, baseado em [3], estudamos as relações existentes entre convergência de funcionais lineares contínuos e convergência de seus conjuntos de nível.

No Capítulo 4, desenvolvemos o estudo de aplicaçoes multilineares, de polinômios e de suas convergências, provando alguns resultados básicos dessa teoria. Estuda-se também, de forma bastante superficial, a diferenciabilidade de polinômios.

No Capítulo 5, baseado em [8], algumas relações estudadas no capítulo 3 são estendidas para o contexto de polinômios homogêneos em espaços de Banach. Além disso, estudam-se relações que ocorrem apenas nesse contexto. 


\section{Notações}

Ao longo desse trabalho, as seguintes notações serão utilizadas:

$\mathbb{N}_{0}$ : o conjunto dos números naturais;

$\mathbb{N}$ : o conjunto dos números naturais positivos;

$\mathbb{K}:$ o corpo $\mathbb{R}$ ou $\mathbb{C}$

$B(x, \epsilon)$ : a bola aberta de centro em $x$ e raio $\epsilon$;

$\overline{B(x, \epsilon)}$ : a bola fechada de centro em $x$ e raio $\epsilon$, em um espaço métrico;

$B[x, \epsilon]$ : a bola fechada de centro em $x$ e raio $\epsilon$, em um espaço normado;

$D(x, \epsilon)$ : o disco aberto no plano complexo de centro em $x$ e raio $\epsilon$;

$e_{n}:$ a seqüência $(0,0, \cdots, 0, \overbrace{1}^{n}, 0, \cdots)$;

$V^{\star}$ : espaço dual de um espaço vetorial $V$;

$E^{\prime}:$ espaço dual de um espaço normado $E$;

$E^{\prime \prime}$ : espaço bidual de um espaço normado $E$;

$[x, y]$ : o conjunto $\{(1-\lambda) x+\lambda y: \lambda \in[0,1]\}$ em um espaço normado $E$ sobre $\mathbb{K}$;

$c_{0}(\mathbb{K})$ : o espaço das seqüências em $\mathbb{K}$ convergentes a 0 ;

$\Re(A)$ : parte real do conjunto $A$, dada por $\Re(A)=\{\Re(z): z \in A\}$, onde $\Re(z)$ denota a parte real de $z \in A$. 


\section{Capítulo 1}

\section{Preliminares}

Nesse capítulo, apresentaremos alguns fatos relevantes sobre espaços de Banach, que serão utilizados ao longo da dissertação.

\subsection{Estruturas em Espaços Vetoriais}

Nessa seção, introduziremos o conceito de norma em um espaço vetorial, e de convergência em norma nesse espaço. Também apresentaremos alguns resultados sobre hiperplanos, que serão utilizados no capítulo 3 .

Seja $E$ um espaço vetorial sobre $\mathbb{K}$. Vamos definir a noção de norma sobre esse espaço. Como passo intermediário, definiremos a noção de semi-norma sobre $E$.

Definição 1.1. Uma semi-norma sobre $E$ é uma função $p: E \rightarrow \mathbb{R}$ que satisfaz as seguintes propriedades:

i) $p(x) \geq 0, \forall x \in E$

ii) $p(\lambda x)=|\lambda| p(x), \forall \lambda \in \mathbb{K}, \forall x \in E$;

iii) $p(x+y) \leq p(x)+p(y), \forall x, y \in E$.

Uma norma sobre $E$ é uma função $\|\cdot\|: E \longrightarrow \mathbb{R}$ que satisfaz as propriedades acima e, além delas, a propriedade seguinte:

$$
\|x\|=0 \Rightarrow x=0
$$

O espaço $(E,\|\cdot\|)$ é dito espaço normado. Quando não houver risco de ambigüidade, denotaremos esse espaço apenas por $E$. A norma de $E$ induz, naturalmente, uma topologia sobre $E$, conhecida por topologia da norma. 
Ao longo dessa dissertação, a seguinte notação será utilizada: se $\left(x_{n}\right)_{n \in \mathbb{N}} \subseteq E$ converge a $x \in E$ na topologia da norma, escreveremos $x=\mathrm{s}-\lim _{n \rightarrow \infty} x_{n}$ ou $x_{n} \stackrel{\|\cdot\|}{\longrightarrow} x$.

Cabe observar que o item iii) de sua definição garante que a norma é uma função convexa. Uma norma em $E$ é dita estritamente convexa quando, para todos $x, y \in E \operatorname{com} x \neq y$ e $\|x\|=\|y\|=1$, tem-se $\|x+y\|<2$. Nesse caso, diz-se que o espaço normado $E$ é estritamente convexo.

A seguir, apresentaremos a definição de hiperplano, e alguns resultados que serão utilizados mais adiante. Cabe ressaltar que a definição pode ser dada para espaços vetoriais em geral.

Definição 1.2. Seja $V$ um espaço vetorial não-nulo. Um hiperplano de $V$ é um subespaço próprio $W \subset V$ com a seguinte propriedade: se $\tilde{W}$ for um subespaço de $V$ com $W \subseteq \tilde{W} \subseteq V$, então $W=\tilde{W}$ ou $\tilde{W}=V$.

Exemplo 1.3. Se $V$ é um espaço vetorial sobre $\mathbb{K}$ com $\operatorname{dim}_{\mathbb{K}} V=n<+\infty$, então todo subespaço de $V$ com dimensão $n-1$ é um hiperplano de $V$. Pode-se mostrar (vide [5]) que a recíproca desse resultado é verdadeira.

O teorema seguinte caracteriza os hiperplanos em espaços de dimensão qualquer. Sua demonstração será omitida. Como referência, podemos citar [5].

Teorema 1.4. Seja $V$ um espaço vetorial sobre $\mathbb{K}$. Se $f \in V^{\star}$ é um funcional linear não-nulo, então ker $f$ é um hiperplano de $V$. Reciprocamente, se $H$ é um hiperplano de $V$, então existe um funcional linear não-nulo $f \in V^{\star}$ tal que ker $f=H$.

Corolário 1.5. Seja $V$ um espaço vetorial sobre $\mathbb{K}$, e sejam $f, g \in V^{\star}$. Então:

$$
\operatorname{ker} f=\operatorname{ker} g \Leftrightarrow \exists \lambda \in \mathbb{K} \text { tal que } f=\lambda g \text {. }
$$

Demonstração. Se existe $\lambda \in \mathbb{K}$ tal que $f=\lambda g$, então é claro que ker $f=\operatorname{ker} g$. Reciprocamente, observe que, se algum dos funcionais for nulo, o outro também deve ser, e não há o que demonstrar. Suponhamos, então, que $f$ e $g$ não são nulos.

Como ker $f$ é um hiperplano, existe $v_{0} \in V$ tal que $V=\operatorname{ker} f+\left[v_{0}\right]$. Então, se $x \in V$, $x=x_{0}+\alpha v_{0}$, com $x_{0} \in \operatorname{ker} f$ e $\alpha \in \mathbb{K}$. Portanto:

$$
f(x)=f\left(x_{0}+\alpha v_{0}\right)=\alpha f\left(v_{0}\right) \quad \text { e } \quad g(x)=g\left(x_{0}+\alpha v_{0}\right)=\alpha g\left(v_{0}\right) .
$$

Como $g\left(v_{0}\right) \neq 0$, podemos tomar:

$$
\lambda=\frac{f\left(v_{0}\right)}{g\left(v_{0}\right)} .
$$

Temos, então, $f(x)=\lambda g(x), \forall x \in V$. 
Observação 1.6. É possível generalizar esse resultado, da seguinte forma:

Sejam $f_{1}, f_{2}, \cdots, f_{n}, g$ funcionais lineares em $V$. Então, $g=\alpha_{1} f_{1}+\cdots+\alpha_{n} f_{n}, \operatorname{com} \alpha_{i} \in \mathbb{K}, 1 \leq$ $i \leq n$, se e somente se $\bigcap_{i=1}^{n} \operatorname{ker}\left(f_{i}\right) \subseteq \operatorname{ker}(g)$.

No entanto, o resultado mais simples, dado pelo lema 1.5 , é suficiente ao nosso contexto.

\subsection{Teoremas Fundamentais e Espaços Reflexivos}

Nessa seção, apresentaremos dois dos teoremas fundamentais da Análise Funcional, que serão utilizados ao longo dessa dissertação. As demonstrações dos resultados principais serão omitidas, por fugirem ao escopo desse trabalho. Como referência, podemos citar os livros [9] e [12].

Cabe ressaltar que, quando nos referirmos ao espaço dual de um espaço normado $E$, estaremos trabalhando com a seguinte norma:

$$
\|f\|=\sup _{\|x\|=1}|f(x)| .
$$

A seguir, apresentaremos o teorema de Hahn-Banach. Esse teorema trata da extensão de funcionais lineares, e é um dos principais teoremas da Análise Funcional. Grande parte de suas aplicações não se dá de forma direta, e sim a partir de seus corolários.

Teorema 1.7. (Hahn-Banach) Sejam $E$ um espaço vetorial sobre $\mathbb{K}, p: E \longrightarrow \mathbb{K}$ uma seminorma em $E, M \subseteq E$ um subespaço próprio de $E$ e $\varphi: M \longrightarrow \mathbb{K}$ um funcional linear tal que $|\varphi(x)| \leq p(x), \forall x \in M$. Então, existe $\bar{\varphi} \in E^{\prime}$ tal que:

1. $\bar{\varphi}_{\mid M}=\varphi ;$

2. $|\bar{\varphi}(x)| \leq p(x), \forall x \in E$.

O corolário seguinte garante que, em um espaço normado não-trivial, sempre existe um funcional linear que separa pontos desse espaço.

Corolário 1.8. Sejam $E$ um espaço normado sobre $\mathbb{K}$ e $x_{0} \in E$, com $x_{0} \neq 0$. Então, existe $\phi \in E^{\prime}$ tal que $\|\phi\|=1$ e $\phi\left(x_{0}\right)=\left\|x_{0}\right\|$.

Demonstração. Seja $M$ o subespaço gerado por $x_{0}$, isto é, $M=\left[x_{0}\right]$, e considere $\varphi: M \longrightarrow \mathbb{K}$ dada por $\varphi\left(\lambda x_{0}\right)=\lambda\left\|x_{0}\right\|, \operatorname{com} \lambda \in \mathbb{K}$. É fácil ver que $\varphi$ é linear.

Além disso, $\left|\varphi\left(\lambda x_{0}\right)\right|=|\lambda|\left\|x_{0}\right\|=\left\|\lambda x_{0}\right\|$, donde $\forall x \in M,|\varphi(x)|=\|x\|$. Logo, $\varphi$ é contínuo e $\|\varphi\|=1$. 
Por Hahn-Banach, existe $\phi \in E^{\prime}$ tal que $\phi_{\mid M I}=\varphi$ e $|\phi(x)| \leq\|x\|, \forall x \in E$. Mas:

$$
\|\phi\|=\sup _{\substack{x \in E \\\|x\|=1}}|\phi(x)| \geq \sup _{\substack{x \in M \\\|x\|=1}}|\phi(x)|=\sup _{\substack{x \in M \\\|x\|=1}}|\varphi(x)|=\|\varphi\|=1 .
$$

Logo, $\|\phi\|=1$ e $\phi\left(x_{0}\right)=\varphi\left(x_{0}\right)=\left\|x_{0}\right\|$.

O corolário seguinte apresenta uma outra forma para a obtenção da norma de um vetor no espaço normado $E$.

Corolário 1.9. Seja $E$ um espaço normado. Para cada $x \in E$, vale que:

$$
\|x\|=\sup _{\|\varphi\|=1}|\varphi(x)|
$$

Demonstração. Seja $x \in E$. Para cada $\varphi \in E^{\prime}$, tem-se $|\varphi(x)| \leq\|\varphi\|\|x\|$. Então:

$$
\sup _{\|\varphi\|=1}|\varphi(x)| \leq\|x\|
$$

Se $x \neq 0$, pelo corolário anterior existe $\phi \in E^{\prime}$ tal que $\|\phi\|=1$ e $\phi(x)=\|x\|$. Logo, $\sup _{\|\varphi\|=1}|\varphi(x)|=$ $\|x\|$. Se $x=0$, a igualdade é trivial.

Finalmente, o próximo corolário nos diz que existe um funcional linear que fornece a distância de um ponto a um subespaço próprio fechado do espaço em questão.

Corolário 1.10. Sejam $E$ um espaço normado e $F \subseteq E$ um subespaço próprio e fechado de $E$. Seja $x_{0} \in E \backslash F$. Então, existe $f: E \rightarrow \mathbb{K}$ tal que:

1. $\|f\|=1$;

2. $f(x)=0, \forall x \in F$;

3. $f\left(x_{0}\right)=\inf _{x \in F}\left\|x_{0}-x\right\|=d\left(x_{0}, F\right)$.

Demonstração. Vamos definir $M=\left[x_{0}, F\right]=\left\{x+\lambda x_{0}: x \in F, \lambda \in \mathbb{K}\right\}$ e $\varphi: M \longrightarrow \mathbb{K}$ dada por $\varphi\left(x+\lambda x_{0}\right)=\lambda \delta$, onde $\delta=d\left(x_{0}, F\right)$. É fácil ver que $\varphi$ está bem definida e é linear.

Se $x \in F$, então $\lambda=0$ e $\varphi(x)=0$. Além disso, $\varphi\left(x_{0}\right)=\delta$. Vamos, agora, mostrar que $\varphi$ é contínuo. Se $\lambda \neq 0$, temos:

$$
\left\|x+\lambda x_{0}\right\|=|\lambda|\left\|\frac{x}{\lambda}+x_{0}\right\| \geq|\lambda| \delta=\left|\varphi\left(x+\lambda x_{0}\right)\right| .
$$


Se $\lambda=0$, a desigualdade é imediata. Logo, $\varphi$ é contínuo e $\|\varphi\| \leq 1$. Dado $\epsilon>0$, existe $x \in F$ tal que $\left\|x+x_{0}\right\|<\delta+\epsilon$. Considere $z=\frac{x+x_{0}}{\left\|x+x_{0}\right\|}$. Temos:

$$
\varphi(z)=\frac{1}{\left\|x+x_{0}\right\|} \varphi\left(x+x_{0}\right)=\frac{\delta}{\left\|x+x_{0}\right\|}>\frac{\delta}{\delta+\epsilon} .
$$

Fazendo $\epsilon \rightarrow 0$, temos que $|\varphi(z)| \geq 1$. Então, $\|\varphi\|=1$. Por Hahn-Banach, existe $f: E \longrightarrow \mathbb{K}$ tal que $f_{\mid M} \equiv 0, f\left(x_{0}\right)=\varphi\left(x_{0}\right)=\delta$ e $\|f\|=\|\varphi\|=1$.

O próximo teorema é conhecido como Princípio da Limitação Uniforme, ou Teorema de BanachSteinhauss. Esse teorema permite concluir, a partir da limitação pontual de uma seqüência de operadores, a sua limitação uniforme.

Teorema 1.11. (Banach-Steinhauss) Sejam $E$ um espaço de Banach e $F$ um espaço normado. Seja $\left(T_{n}\right)_{n \in \mathbb{N}}$ uma seqüência de operadores de $E$ em $F$ tal que, para cada $x \in E$, existe $M_{x} \in \mathbb{R}$ que satisfaz:

$$
\left\|T_{n}(x)\right\| \leq M_{x}, \forall n \in \mathbb{N} .
$$

Então, existe $M \in \mathbb{R}$ tal que $\left\|T_{n}\right\| \leq M, \forall n \in \mathbb{N}$.

A seguir, apresentaremos o conceito de espaço reflexivo, que será utilizado no estudo das relações entre convergências de funcionais e convergência de conjuntos no sentido de Mosco.

Seja $E$ um espaço normado sobre $\mathbb{K}$. Dado $x \in E$, podemos definir o funcional linear contínuo $g_{x}: E^{\prime} \longrightarrow \mathbb{K}$ por $g_{x}(f)=f(x)$. Por Hahn-Banach,

$$
\left\|g_{x}\right\|=\sup _{\|f\|=1}\left|g_{x}(f)\right|=\sup _{\|f\|=1}|f(x)|=\|x\| .
$$

Seja $E$ um espaço normado sobre $\mathbb{K}$. A aplicação canônica entre $E$ e seu bidual $E^{\prime \prime}$ é a aplicação $C: E \longrightarrow E^{\prime \prime}$ dada por $C(x)=g_{x}$. É fácil ver que $C$ é linear. Além disso, $C$ é um isomorfismo entre $E$ e $C(E)$, uma vez que $g_{x}-g_{y}=g_{x-y}$ e, portanto, $\left\|g_{x}-g_{y}\right\|=\left\|g_{x-y}\right\|=\|x-y\|$.

Vamos, agora, definir a reflexividade de um espaço normado.

Definição 1.12. O espaço normado $E$ é reflexivo $\operatorname{se} \operatorname{Im}(C)=E^{\prime \prime}$.

Exemplo 1.13. Os exemplos seguintes apresentam algumas classes de espaços reflexivos:

a) Todo espaço de dimensão finita é reflexivo;

b) Os espaços $l_{p}(\mathbb{K})=\left\{x=\left(x_{n}\right)_{n \in \mathbb{N}} \subset \mathbb{K}: \sum_{n=1}^{+\infty}\left|x_{n}\right|^{p}<+\infty\right\}$, com $1<p<+\infty$, são reflexivos.

As demonstrações desses fatos podem ser encontradas em [9]. 
Teorema 1.14. Se E é um espaço normado reflexivo, então E é um espaço de Banach.

Demonstração. Como $E^{\prime}$ é um espaço normado e $\mathbb{K}$ é completo, a teoria de funcionais nos garante que $E^{\prime \prime}$ é um espaço de Banach. Como $E$ é reflexivo, segue que $E$ é isomorfo a $E^{\prime \prime}$. Logo, $E$ é um espaço de Banach.

\subsection{Topologias Fraca e Fraca- $\star$}

Essa seção será dedicada a apresentar as topologias fraca e fraca- $\star$. Além disso, definiremos a convergência com relação a cada uma delas, e compararemos essas definições entre si e com a convergência em norma.

Inicialmente, vamos definir a topologia fraca em $E$ :

Definição 1.15. Seja $G$ um subconjunto de $E$. Dizemos que $G$ é fracamente aberto se, para cada $x \in G$, existem $f_{1}, \cdots, f_{n} \in E^{\prime} e \epsilon>0$ tais que:

$$
U\left(x, f_{1}, \cdots, f_{n}, \epsilon\right)=\left\{y \in E: \sup _{1 \leq i \leq n}\left|f_{i}(y-x)\right|<\epsilon\right\} \subset G .
$$

A topologia fraca em $E$ é a topologia formada pela família dos conjuntos fracamente abertos em $E$, unida com o conjunto vazio.

Essa topologia dá origem a um tipo de convergência conhecido como convergência fraca. Vamos definir esse tipo de convergência:

Definição 1.16. Seja $E$ um espaço normado sobre $\mathbb{K}$. Uma seqüência $\left(x_{n}\right)_{n \in \mathbb{N}} \subset E$ é dita fracamente convergente em $E$ se existe $x \in E$ tal que, para cada $f \in E^{\prime}, \lim _{n \rightarrow \infty} f\left(x_{n}\right)=f(x)$.

Denotaremos essa convergência por $x=\mathrm{w}-\lim _{n \rightarrow \infty} x_{n}$ ou $x_{n} \stackrel{\mathrm{w}}{\longrightarrow} x$.

O exemplo seguinte mostra que a topologia fraca pode estar propriamente contida na topologia da norma:

Exemplo 1.17. Considere a seqüência $\left(e_{n}\right)_{n \in \mathbb{N}} \subset l_{2}(\mathbb{K})$. Temos:

1. Se $f \in l_{2}^{\prime}(\mathbb{K})$, então $f\left(e_{n}\right) \rightarrow 0$. Logo, $\left(e_{n}\right)_{n \in \mathbb{N}}$ converge a 0 na topologia fraca;

2. Como $\left\|e_{n}\right\|=1, \forall n \in \mathbb{N}$, a seqüência $\left(e_{n}\right)_{n \in \mathbb{N}}$ não converge a 0 na topologia da norma. 
A topologia fraca em $E$ é a menor topologia para a qual cada elemento de $E^{\prime}$ é contínuo. Dessa forma, essa topologia deve ser menos fina que qualquer topologia na qual todos os elementos de $E^{\prime}$ sejam contínuos. Em particular, a topologia fraca é menos fina que a topologia da norma. Portanto, podemos enunciar a seguinte:

Proposição 1.18. Sejam $E$ um espaço normado e $f$ um funcional linear em $E$. Então, $f$ é contínuo com respeito à topologia fraca se e somente se é contínuo com respeito à topologia da norma.

O seguinte lema apresenta alguns fatos sobre a convergência fraca.

Lema 1.19. Seja $E$ um espaço normado, e seja $\left(x_{n}\right)_{n \in \mathbb{N}} \subset E$ tal que $x_{n} \stackrel{\text { w }}{\longrightarrow} x \in E$. Então:

1. O limite de $\left(x_{n}\right)_{n \in \mathbb{N}}$ é único;

2. A seqüência $\left(\left\|x_{n}\right\|\right)_{n \in \mathbb{N}}$ é limitada.

Demonstração. (1) Suponha que $x_{n} \stackrel{\text { w }}{\longrightarrow} x$ e que $x_{n} \stackrel{\text { w }}{\longrightarrow} y$. Se $f \in E^{\prime}$, então $f\left(x_{n}\right) \rightarrow f(x)$ e $f\left(x_{n}\right) \rightarrow f(y)$. Logo, $f(x)=f(y)$, e $f(x-y)=0$.

Como $f$ é arbitrária, segue, pelo corolário 1.9 , que $x-y=0$.

(2) Para cada $f \in E^{\prime},\left(f\left(x_{n}\right)\right)_{n \in \mathbb{N}} \subseteq \mathbb{K}$ é convergente e, portanto, limitada. Utilizando a aplicação canônica $C: E \longrightarrow E^{\prime \prime}$, definimos $g_{n}(f)=f\left(x_{n}\right), \forall n \in \mathbb{N}$. Assim, a seqüência $\left(\left|g_{n}(f)\right|\right)_{n \in \mathbb{N}}$ é limitada, $\forall f \in E^{\prime}$.

Mas $E^{\prime}$ é completo. Então, pelo teorema 1.11, $\left(\left\|g_{n}\right\|\right)_{n \in \mathbb{N}}$ é limitada. Como $\left\|g_{n}\right\|=\left\|x_{n}\right\|$, segue o resultado.

O teorema seguinte compara os dois tipos de convergência já definidos:

Teorema 1.20. Seja $E$ um espaço normado, e seja $\left(x_{n}\right)_{n \in \mathbb{N}} \subseteq E$ uma seqüência. Então,

1. Se $x_{n} \stackrel{\|\cdot\|}{\longrightarrow} x$, então $x_{n} \stackrel{w}{\longrightarrow} x$;

2. Se $\operatorname{dim}_{\mathbb{K}} E<+\infty$, as duas noções de convergência são equivalentes.

Demonstração. (1) Se $x_{n} \stackrel{\|\cdot\|}{\longrightarrow} x$, então $\left\|x_{n}-x\right\| \rightarrow 0$. Tome $f \in E^{\prime}$. Temos:

$$
\left|f\left(x_{n}\right)-f(x)\right|=\left|f\left(x_{n}-x\right)\right| \leq\|f\|\left\|x_{n}-x\right\| .
$$

Como $f$ era arbitrária, segue que $x_{n} \stackrel{\mathrm{w}}{\longrightarrow} x$. 
(2) É necessário provar que a convergência fraca da seqüência implica sua convergência em norma. Suponhamos que $x_{n} \stackrel{\mathrm{w}}{\longrightarrow} x$ e que $\operatorname{dim}_{\mathbb{K}} E=k \in \mathbb{N}$.

Seja $\left\{e_{1}, e_{2}, \cdots, e_{k}\right\}$ uma base de $E$, e escrevamos $x_{n}=\sum_{i=1}^{k} \alpha_{i}^{n} e_{i}$ e $x=\sum_{i=1}^{k} \alpha_{i} e_{i}$. Por hipótese, $f\left(x_{n}\right) \rightarrow f(x), \forall f \in E^{\prime}$.

Tome, em particular, $f_{1}, \cdots, f_{k}$ dadas por $f_{i}\left(e_{j}\right)=\delta_{i j}, 1 \leq i, j \leq k$. Então, $f_{i}\left(x_{n}\right)=\alpha_{i}^{n}$ e $f_{i}(x)=\alpha_{i}$. Logo, $\alpha_{i}^{n} \rightarrow \alpha_{i}, \forall 1 \leq i \leq k$.

Obtemos:

$$
\left\|x_{n}-x\right\|=\left\|\sum_{i=1}^{k}\left(\alpha_{i}^{n}-\alpha_{i}\right) e_{i}\right\| \leq \sum_{i=1}^{k} \mid \alpha_{i}^{n}-\alpha_{i}\left\|e_{i}\right\| \rightarrow 0
$$

e $x_{n} \stackrel{\|\cdot\|}{\longrightarrow} x$

Cabe ressaltar que existem espaços de dimensão infinita onde essas noções de convergência também são equivalentes. Em 1921, Schur ([16]) mostrou que isso acontece em $l_{1}(\mathbb{K})$.

No que segue, vamos definir a topologia fraca- $\star$ de funcionais em $E^{\prime}$.

Definição 1.21. Seja $G$ um subconjunto de $E^{\prime}$. Dizemos que $G$ é fraca-» aberto se, para cada $g \in G$, existem $x_{1}, \cdots, x_{n} \in E$ e $\epsilon>0$ tais que:

$$
V\left(g, x_{1}, \cdots, x_{n}, \epsilon\right)=\left\{f \in E^{\prime}: \sup _{1 \leq i \leq n}\left|f\left(x_{i}\right)-g\left(x_{i}\right)\right|<\epsilon\right\} \subset G .
$$

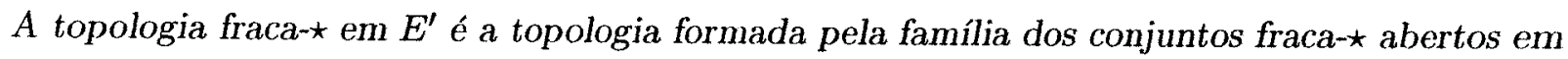
$E$, unida com o conjunto vazio.

Essa topologia dá origem à convergência fraca-^ de funcionais, definida a seguir:

Definição 1.22. Seja $E$ um espaço normado e seja $\left(f_{n}\right)_{n \in \mathbb{N}}$ uma seqüência de funcionais em $E^{\prime}$. A convergência fraca- $\star$ dessa seqüência ocorre se existe $f \in E^{\prime}$ tal que $f_{n}(x) \rightarrow f(x), \forall x \in E$.

Nesse caso, escrevemos $f=\mathrm{w}^{\star}-\lim _{n \rightarrow \infty} f_{n}$ ou $f_{n} \stackrel{\mathrm{w}^{\star}}{\longrightarrow} f$.

O exemplo seguinte mostra que a convergência fraca-^ não implica, necessariamente, a convergência fraca de uma seqüência de funcionais.

Exemplo 1.23. Sabemos que $c_{0}^{\prime}(\mathbb{K})=l_{1}(\mathbb{K})$. Considere a sequiência $\left(e_{n}^{\prime}\right)_{n \in \mathbb{N}} \subset c_{0}^{\prime}(\mathbb{K})$ de elementos que correspondem, de forma canônica, aos elementos $\left(e_{n}\right)_{n \in \mathbb{N}}$ de $l_{1}(\mathbb{K})$.

Vamos mostrar que $\left(e_{n}^{\prime}\right)_{n \in \mathbb{N}}$ converge fraca- $\star$ a 0 . Tome $x \in c_{0}(\mathbb{K})$. Se $x=\left(x_{n}\right)_{n \in \mathbb{N}}$, então $x_{n} \rightarrow 0$. Mas $e_{n}^{\prime}(x)=x_{n} \rightarrow 0$, donde $e_{n}^{\prime} \stackrel{\mathrm{w}^{*}}{\longrightarrow} 0$. 
Mas $\left(e_{n}^{\prime}\right)_{n \in \mathbb{N}}$ não converge fracamente a 0 . De fato, sabemos que $c_{0}^{\prime \prime}(\mathbb{K})=l_{1}^{\prime}(\mathbb{K})=l_{\infty}(\mathbb{K})$, o espaço das seqüências limitadas. Tome $g \in c_{0}^{\prime \prime}(\mathbb{K})$ o elemento associado a $(1,1,1, \cdots) \in l_{\infty}(\mathbb{K})$. Então, $g\left(e_{n}^{\prime}\right)=1, \forall n \in \mathbb{N}$, e segue o resultado.

A topologia fraca- $\star$ de $E^{\prime}$ é a menor topologia em $E^{\prime}$ na qual se tem que, para cada $x \in E$, o funcional linear em $E^{\prime}$ dado por $f \ni E \mapsto f(x)$ é contínuo.

Como $C(E) \subseteq E^{\prime \prime}$ e a topologia fraca- $\star$ em $E^{\prime}$ é a menor topologia na qual todos os elementos de $E^{\prime \prime}$ são contínuos, a topologia fraca- $\star$ em $E^{\prime}$ é menos fina que a topologia fraca em $E^{\prime}$. Além disso, ambas as topologias coincidem se e somente se $C(E)=E^{\prime \prime}$, isto é, se $E$ é reflexivo. Esses fatos serão registrados no teorema seguinte, que relaciona as convergência fraca e fraca- $\star$ de funcionais:

Teorema 1.24. Seja $E$ um espaço normado e seja $\left(f_{n}\right)_{n \in \mathbb{N}}$ uma seqüência de funcionais em $E^{\prime}$. Então:

1. Se $f_{n} \stackrel{\mathrm{w}}{\longrightarrow} f$, então $f_{n} \stackrel{\mathrm{w}^{*}}{\longrightarrow} f$;

2. Se E é reflexivo, a recíproca de (1) é verdadeira.

Demonstração. (1) Suponha que $f_{n} \stackrel{\text { w }}{\longrightarrow} f$. Então, $\forall g \in E^{\prime \prime}, g\left(f_{n}\right) \rightarrow g(f)$. Para cada $x \in E$ e cada $f \in E^{\prime}$, seja $g_{x}(f)=f(x)$. Já vimos que $g_{x} \in E^{\prime \prime}$. Então, $\forall x \in E, f_{n}(x) \rightarrow f(x)$, donde $f_{n} \stackrel{\mathrm{w}^{*}}{\longrightarrow} f$.

(2) Suponhamos $E$ reflexivo e $f_{n} \stackrel{w^{*}}{\longrightarrow} f$. Então, $\forall x \in E, f_{n}(x) \rightarrow f(x)$. Como $E$ é reflexivo, a aplicação canônica $C$ é bijetora. Seja $g \in E^{\prime \prime}$. Então, $g=g_{x_{0}}$, para algum $x_{0} \in E$. Mas $f_{n}\left(x_{0}\right) \rightarrow f\left(x_{0}\right)$, donde segue que $g\left(f_{n}\right) \rightarrow g(f)$. Como $g$ era arbitrário, segue que $f_{n} \stackrel{\text { w }}{\longrightarrow} f$.

Corolário 1.25. Seja $E$ um espaço normado e seja $\left(f_{n}\right)_{n \in \mathbb{N}}$ uma seqüência de funcionais em $E^{\prime}$. Então:

1. Se $f_{n} \stackrel{\|\|}{\longrightarrow} f$, então $f_{n} \stackrel{w^{*}}{\longrightarrow} f$;

2. Se $\operatorname{dim}_{\mathbb{K}} E<+\infty$, as três noções de convergência são equivalentes.

Demonstração. (1) Trivial, pois convergência em norma implica convergência fraca.

(2) Se $\operatorname{dim}_{\mathrm{K}} E<+\infty$, as convergências em norma e fraca são equivalentes. Além disso, $E$ é reflexivo, donde as convergências fraca e fraca-^ são equivalentes. 


\section{Capítulo 2}

\section{Convergência de Conjuntos}

Ao longo desse capítulo, apresentaremos as noções de convergência de conjuntos utilizadas ao longo dessa dissertação. Em geral, utilizaremos essas noções apenas em espaços normados. No entanto, a maior parte delas pode ser introduzida no contexto de espaços métricos em geral. A primeira parte da teoria foi desenvolvida com base no livro [10].

Antes de adentrar à teoria, devemos mencionar uma notação que será largamente utilizada ao longo desse texto. Se $N \subseteq \mathbb{N}$ é um subconjunto infinito de $\mathbb{N}$, a notação $\left(a_{n}\right)_{n \in N}$ indica a seqüência:

$$
a_{n(1)}, a_{n(2)}, a_{n(3)}, a_{n(4)}, \cdots
$$

onde $n(i)$ é o i-ésimo natural em $N$, em termos da ordem usual de $\mathbb{N}$. As noções de limite e de convergência, em função dessa notação, podem ser facilmente entendidas.

No que segue, também utilizaremos a seguinte notação: se $A$ e $B$ são subconjuntos de um espaço vetorial $V$ sobre $\mathbb{K}$ e $r \in \mathbb{K}$, então:

$$
A+r B=\{a+r b: a \in A, b \in B\}
$$

\subsection{O Espaço $C L(X)$}

Considere $(X, d)$ um espaço métrico. Quando não houver perigo de ambigüidade, denotaremos esse espaço apenas por $X$. Vamos denotar por $C L(X)$ o conjunto de todos os subconjuntos fechados de $X$, isto é,

$$
C L(X)=\{S \subseteq X: S \text { é fechado }\}
$$

O espaço $C L(X)$ é tradicionalmente munido de uma topologia, conhecida como topologia exponencial. Para definirmos essa topologia, é necessário, primeiramente, definirmos o significado de $C L(A), \operatorname{com} A \subset X$. 
$\mathrm{O}$ conjunto $C L(A)$ é formado por todos os subconjuntos de $A$ que são fechados em $X$. Evidentemente, se um conjunto $S \subseteq A$ é fechado em $X$, também o é em $A$, uma vez que se pode escrever $S=S \cap A$. A seguir, definimos a topologia exponencial em $C L(X)$.

Definição 2.1. A topologia exponencial é a menor topologia em $C L(X)$ para a qual vale:

i) Se $A$ é aberto, $C L(A)$ é aberto;

ii) Se $A$ é fechado, $C L(A)$ é fechado.

Uma sub-base aberta para essa topologia é a família de conjuntos:

$$
\Im=\{C L(G): G \subset X \text { aberto }\} \cup\{C L(X) \backslash C L(X \backslash G): G \subset X \text { aberto }\}
$$

Utilizaremos essa topologia apenas para definir alguns tipos de convergência em $C L(X)$. Port.anto, não estaremos interessados em suas propriedades, que são detalhadamente apresentadas em $[10]$.

\subsection{A distância de Hausdorff}

Nessa seção, discutiremos a definição de distância de Hausdorff. Para cada $A \in C L(X)$, com $A \neq \emptyset$, podemos definir, em $X$, a função distância $d(\cdot, A): X \rightarrow \mathbb{R}$ de um ponto $x \in X$ ao conjunto $A$, dada por:

$$
d(x, A)=\inf _{a \in A} d(x, a) .
$$

Por convenção, adotaremos $d(x, \emptyset)=+\infty, \forall x \in X$. Uma das mais familiares noções de convergência de conjuntos fechados é a convergência com relação à distância de Hausdorff. Essa distância é denotada por $h_{d}$ e definida, em $(C L(X) \backslash\{\emptyset\}) \times(C L(X) \backslash\{\emptyset\})$, por:

$$
h_{d}(A, B)=\sup (\{d(a, B): a \in A\} \cup\{d(b, A): b \in B\}) .
$$

Observe que $h_{d}(A, A)=0$ e que $h_{d}(A, B)=h_{d}(B, A)$. No entanto, o exemplo abaixo mostra que o fato de a distância de Hausdorff entre dois conjuntos ser mula não implica a igualdade entre esses conjuntos.

Exemplo 2.2. Considere $X=\mathbb{R}, A=[-1,1]$ e $B=[-1,0) \cup(0,1]$. Temos:

- $d(a, B)=0, \forall a \in A$;

- $d(b, A)=0, \forall b \in B$. 
Logo, $h_{d}(A, B)=0$, embora tenhamos $A \neq B$. No entanto, é fácil ver que $h_{d}(A, B)=0$ implica $A \subseteq B$ ou $B \subseteq A$.

O seguinte lema relaciona a distância de Hausdorff à distância dos pontos do espaço aos conjuntos em questão:

Lema 2.3. Seja $X$ um espaço métrico, e sejam $A, B \in C L(X)$ dois conjuntos não-vazios. Então,

$$
h_{d}(A, B)=\sup _{x \in X}|d(x, A)-d(x, B)| .
$$

Demonstração. Observemos que:

$$
\{d(a, B): a \in A\},\{d(b, A): b \in B\} \subseteq\{|d(x, A)-d(x, B)|: x \in X\} .
$$

Dessa forma:

$$
(\{d(a, B): a \in A\} \cup\{d(b, A): b \in B\}) \subseteq\{|d(x, A)-d(x, B)|: x \in X\} .
$$

Logo, $h_{d}(A, B) \leq \sup _{x \in X}|d(x, A)-d(x, B)|$.

Suponhamos agora, por absurdo, que $h_{d}(A, B)<\sup _{x \in X}|d(x, A)-d(x, B)|$. Então, existe $x_{0} \in$ $X \backslash(A \cup B)$ tal que:

$$
\left|d\left(x_{0}, A\right)-d\left(x_{0}, B\right)\right|>\sup (\{d(a, B): a \in A\} \cup\{d(b, A): b \in B\}) .
$$

Se $d\left(x_{0}, A\right) \geq d\left(x_{0}, B\right)$, temos:

$$
d\left(x_{0}, A\right)>\sup (\{d(a, B): a \in A\} \cup\{d(b, A): b \in B\})+d\left(x_{0}, B\right) .
$$

Fixe $a_{0} \in A$ arbitrário. Então, $d\left(x_{0}, A\right)>d\left(a_{0}, B\right)+d\left(x_{0}, B\right)$. Observe, agora, que os conjuntos $\left\{d\left(a_{0}, b\right): b \in B\right\}$ e $\left\{d\left(x_{0}, b\right): b \in B\right\}$ são limitados inferiormente. Portanto:

$$
\inf _{a \in A} d\left(x_{0}, a\right)>\inf _{b \in B} d\left(a_{0}, b\right)+\inf _{b \in B} d\left(x_{0}, b\right)=\inf _{b \in B}\left(d\left(a_{0}, b\right)+d\left(x_{0}, b\right)\right) \geq d\left(a_{0}, x\right),
$$

o que gera uma contradição. Analogamente, chega-se a uma contradição com a hipótese de que $d\left(x_{0}, A\right)<d\left(x_{0}, B\right)$, e o resultado segue.

O seguinte corolário é imediato:

Corolário 2.4. Sejam $X$ um espaço métrico, $\left(A_{n}\right)_{n \in \mathbb{N}}$ uma seqüência de conjuntos não-vazios em $C L(X)$ e $A \in C L(X)$ um conjunto não-vazio. Se $h_{d}\left(A_{n}, A\right) \rightarrow 0$, então $\left(d\left(\cdot, A_{n}\right)\right)_{n \in \mathbb{N}}$ converge uniformemente a $d(\cdot, A)$. 
Em particular, esse corolário garante que a convergência de uma seqüência de conjuntos com relação à distância de Hausdorff implica a convergência pontual de suas funções distância, isto é, se uma seqüência de conjuntos não-vazios $\left(A_{n}\right)_{n \in \mathbb{N}} \subseteq C L(X)$ converge com relação à distância de Hausdorff a um conjunto $A \in C L(X)$, então $\left(d\left(\cdot, A_{n}\right)\right)_{n \in \mathbb{N}}$ converge pontualmente à função $d(\cdot, A)$. Essa noção de convergência será discutida na seção seguinte.

Exemplo 2.5. A seguir, apresentamos dois exemplos de seqüências de conjuntos convergentes com relação à distância de Hausdorff:

a) Para cada $n \in \mathbb{N}$, defina, em $\mathbb{R}$ :

$$
A_{n}=\left[\frac{-1}{n}, \frac{1}{n}\right]
$$

Seja $A=\{0\}$. Afirmamos que $A_{n} \stackrel{h_{d}}{\longrightarrow} A$. De fato, se $x \in \mathbb{R}$, temos dois casos:

Caso I: $x \in A_{n}$, para algum $n \in \mathbb{N}$.

$$
\left|d\left(x, A_{n}\right)-d(x, A)\right|=|0-| x|| \leq \frac{1}{n}
$$

pois, se $x \in A_{n}$, então $|x| \leq \frac{1}{n}$.

Caso II: $x \notin A_{n}, \forall n \in \mathbb{N}$.

$$
\left|d\left(x, A_{n}\right)-d(x, A)\right|=|| x-\frac{1}{n}|-| x|| \leq \frac{1}{n} .
$$

Logo, $h_{d}\left(A_{n}, A\right) \rightarrow 0$ quando $n \rightarrow \infty$.

b) Para cada $n \in \mathbb{N}$, defina, em $\mathbb{R}^{2}$ :

$$
A_{n}=B\left(a, r+\frac{1}{n^{2}}\right), \text { com } a \in \mathbb{R}^{2} \text { e } r>0 .
$$

Seja $A=B(a, r)$. É fácil ver que $h_{d}\left(A_{n}, A\right) \leq \frac{1}{n^{2}}$. Logo, $\left(A_{n}\right)_{n \in \mathbb{N}}$ converge a $A$ com relação à distância de Hausdorff.

\subsection{A Convergência de Wijsman}

Nessa seção, discutiremos a definição de convergência de conjuntos no sentido de Wijsman, e relacionaremos essa definição com a noção de convergência de conjuntos com relação à distância de Hausdorff. 
Definição 2.6. Uma seqüência $\left(A_{n}\right)_{n \in \mathbb{N}} \subseteq C L(X)$ de conjuntos não-vazios é dita convergente no sentido de Wijsman a un conjunto $A \in C L(X)$ se a seqüência de suas funções distância, $\left(d\left(\cdot, A_{n}\right)\right)_{n \in \mathbb{N}}$, converge pontualmente à função $d(\cdot, A)$.

Nesse caso, escrevemos $A=\mathrm{W}-\lim _{n \in \mathbb{N}} A_{n}$.

De acordo com o corolário 2.4, é claro que a convergência com relação à distância de Hausdorff de uma seqüência de conjuntos implica a convergência, no sentido de Wijsman, dessa mesma seqüência.

Exemplo 2.7. Os exemplos exibidos na seção anterior apresentam seqüências convergentes no sentido de Wijsman, uma vez que essas seqüências convergem com relação à distância de Hausdorff.

No entanto, podemos exibir uma seqüência de conjuntos convergente no sentido de Wijsman que não converge com relação à distância de Hausdorff:

Exemplo 2.8. Considere $X=] 0,+\infty[\subset \mathbb{R}$, e sejam:

$$
A=\mathbb{N} \quad \text { e } \quad A_{n}=\{1,2, \cdots, n\}
$$

É fácil ver que $\left(d\left(\cdot, A_{n}\right)\right)_{n \in \mathbb{N}}$ converge pontualmente a $d(\cdot, A)$. No entanto, para cada $n \in \mathbb{N}$, temos:

$$
h_{d}\left(A_{n}, A\right)=\sup _{x \in X}\left|d\left(x, A_{n}\right)-d(x, A)\right|=+\infty .
$$

Logo, $\left(A_{n}\right)_{n \in \mathbb{N}}$ não converge a $A$ com relação à distância de Hausdorff.

Uma pergunta natural que surge a partir desses exemplos é: quando as duas noções de convergência definidas até então são equivalentes? A proposição seguinte nos garante que isso ocorre somente quando o espaço em que estamos trabalhando é totalmente limitado, isto é, quando, para cada $\epsilon>0$, existe um número finito de bolas de raio $\epsilon$ que recobrem o espaço.

Proposição 2.9. Sejam $X$ um espaço métrico, $\left(A_{n}\right)_{n \in \mathbb{N}} \subseteq C L(X)$ uma seqüência de conjuntos não-vazios e $A \in C L(X)$ um conjunto não-vazio. São equivalentes:

1. $X$ é totalmente limitado;

2. $A=\mathrm{W}-\lim _{n \in \mathbb{N}} A_{n} \Leftrightarrow\left(A_{n}\right)_{n \in \mathbb{N}}$ converge a $A$ com relação à distância de Hausdorff.

Demonstração. (1) $\Rightarrow(2)$ Já vimos que, mesmo sem assumirmos nenhuma hipótese adicional sobre o espaço $X$, a convergência com relação à distância de Hausdorff de uma seqüência de conjuntos garante a convergência pontual das funções distância dessa seqüência de conjuntos. 
Suponhamos, então, que $X$ é totalmente limitado e que $\left(d\left(\cdot, A_{n}\right)\right)_{n \in \mathbb{N}}$ converge pontualmente a $d(\cdot, A)$. Sejam $\epsilon>0$ e $F \subseteq X$ um conjunto finito tal que $\forall y \in X, d(y, F) \leq \frac{\epsilon}{3}$. Note que esse conjunto existe, uma vez que $X$ é totalmente limitado. Para cada $x \in F$, escolha $n_{0} \in \mathbb{N}$ suficientemente grande de modo que, se $n>n_{0}$, tenhamos:

$$
\left|d\left(x, A_{n}\right)-d(x, A)\right|<\frac{\epsilon}{3} .
$$

Seja $y \in X$, e fixe $x \in F$. Como as funções distância são lipschitzianas com constante de Lipschitz igual a 1 , temos, para $n>n_{0}$ :

$$
\begin{gathered}
\left|d\left(y, A_{n}\right)-d(y, A)\right|=\left|d\left(y, A_{n}\right)-d\left(x, A_{n}\right)+d\left(x, A_{n}\right)-d(x, A)+d(x, A)-d(y, A)\right| \leq \\
\leq\left|d\left(y, A_{n}\right)-d\left(x, A_{n}\right)\right|+\left|d\left(x, A_{n}\right)-d(x, A)\right|+|d(x, A)-d(y, A)| \leq \\
\leq 2 d(x, y)+\left|d\left(x, A_{n}\right)-d(x, A)\right|<3 \frac{\epsilon}{3}=\epsilon .
\end{gathered}
$$

Como $y \in X$ era arbitrário, segue que $\sup _{y \in X}\left|d\left(y, A_{n}\right)-d(y, A)\right| \leq \epsilon$. Assim, pelo lema 2.3, $\left(A_{n}\right)_{n \in \mathbb{N}}$ converge a $A$ com relação à distância de Hausddorff.

$(2) \Rightarrow(1)$ Suponhamos que $X$ não é totalmente limitado. Usando o Lema de Zorn, podemos afirmar que existem $\epsilon>0$ e $A_{\epsilon}$ infinito tais que:

$$
X \subseteq B_{\epsilon}\left(A_{\epsilon}\right)=\bigcup_{x \in A_{\epsilon}} B(x, \epsilon)
$$

e, se $\{x, y\} \subseteq A_{\epsilon}$, então $d(x, y) \geq \epsilon$. Seja $A=\left(x_{n}\right)_{n \in \mathbb{N}}$ um subconjunto infinito e enumerável de $A_{\epsilon}$. Para cada $n \in \mathbb{N}$, seja:

$$
A_{n}=\left\{x_{j}: j \leq n\right\} .
$$

É fácil ver que $\left(d\left(\cdot, A_{n}\right)\right)_{n \in \mathbb{N}}$ converge pontualmente a $d(\cdot, A)$. No entanto:

$$
h_{d}\left(A_{n}, A\right)=\sup \left(\left\{d\left(a, A_{n}\right): a \in A\right\} \cup\left\{d(y, A): y \in A_{n}\right\}\right)=\sup \left\{d\left(a, A_{n}\right): a \in A\right\},
$$

e $\left(A_{n}\right)_{n \in \mathbb{N}}$ não converge a $A$ com relação à distância de Hausdorff.

\subsection{A convergência de Kuratowski}

Nessa seção, discutiremos a definição de convergência no sentido de Kuratowski, e apresentaremos uma topologia em $C L(X)$ compatível com essa definição. Inicialmente, vamos definir a convergência de Kuratowski conforme concebida originalmente em [10].

Seja $\left(A_{n}\right)_{n \in \mathbb{N}}$ uma seqüência de conjuntos não-vazios em $C L(X)$. Vamos definir os limites inferior e superior dessa sequiência. Nessa definição, o conjunto $V$ sempre denotará uma vizinhança do ponto $x$ em questão. 
Definição 2.10. Seja $\left(A_{n}\right)_{n \in \mathbb{N}}$ uma seqüência de conjuntos não-vazios em $C L(X)$. O limite inferior de $\left(A_{n}\right)_{n \in \mathbb{N}}$ é:

$$
\text { Li } A_{n}=\left\{x \in X: \forall V \subseteq X, \exists n_{0} \in \mathbb{N} \operatorname{com} V \cap A_{n} \neq \emptyset, \forall n>n_{0}\right\}
$$

O limite superior de $\left(A_{n}\right)_{n \in \mathbb{N}}$ é:

$$
\text { Ls } A_{n}=\left\{x \in X: \forall V \subseteq X, V \text { intercepta infinitos termos } A_{n}\right\} .
$$

Exemplo 2.11. A seguir, apresentamos dois exemplos que ilustram essa definição:

a) Seja $X=\mathbb{R}$. Para cada $n \in \mathbb{N}$, defina, em $X$ :

$$
A_{n}=\left[\frac{1}{n}, 1\right], \text { se } n \text { é par e } \quad A_{n}=\left[\frac{1}{2}, 1\right], \text { se } n \text { é ímpar }
$$

Então, Li $A_{n}=\left[\frac{1}{2}, 1\right]$ e Ls $A_{n}=[0,1]$.

b) Seja $X=\mathbb{R}$. Para cada $n \in \mathbb{N}$, defina, em $X$ :

$$
A_{n}=[n, n+1]
$$

Então, Li $A_{n}=\operatorname{Ls} A_{n}=\emptyset$.

O exemplo mostra que não se pode garantir que Li $A_{n}$ e Ls $A_{n}$ sejam não-vazios, mesmo quando a seqüência $\left(A_{n}\right)_{n \in \mathbb{N}}$ é formada apenas por conjuntos não-vazios. Ao longo dessa dissertação, estaremos interessados apenas no caso em que esses limites não são conjuntos vazios. Além disso, é claro que Li $A_{n} \subseteq$ Ls $A_{n}$. O exemplo também mostra que essa inclusão pode ser estrita.

O seguinte lema nos garante que tais conjuntos são fechados e, portanto, estão em $C L(X)$ :

Lema 2.12. Os conjuntos Li $A_{n}$ e Ls $A_{n}$ são fechados em $X$.

Demonstração. Suponhamos $\operatorname{Li} A_{n} \neq \emptyset$. Tome $p \in \overline{\operatorname{Li} A_{n}}$, e seja $W$ uma vizinhança aberta de $p$. Então, existe $q \in W \cap \operatorname{Li} A_{n}$. Como $q \in \operatorname{Li} A_{n}$, existe $n_{0} \in \mathbb{N}$ tal que $n>n_{0} \Rightarrow W \cap A_{n} \neq \emptyset$ e, por definição, $p \in \operatorname{Li} A_{n}$. Analogamente, mostra-se que Ls $A_{n}$ é fechado.

A partir dessas definições, pode-se definir a convergência no sentido de Kuratowski, da seguinte forma:

Definição 2.13. Seja $\left(A_{n}\right)_{n \in \mathbb{N}}$ uma seqüência de conjuntos não-vazios em $C L(X)$. Dizemos que $\left(A_{n}\right)_{n \in \mathbb{N}}$ converge no sentido de Kuratowski a um conjunto $A \in C L(X)$ se $A=\operatorname{Li} A_{n}=\operatorname{Ls} A_{n}$. 
Nesse caso, escrevemos $A=\mathrm{K}-\lim _{n \in \mathbb{N}} A_{n}$.

Da maneira como é colocada, a definição da convergência no sentido de Kuratowski fica abstrata demais para o uso que desejamos fazer dela; a saber, relacioná-la à convergência de funcionais lineares contínuos e de polinômios homogêneos. Dessa forma, as duas proposições seguintes atuam como uma ferramenta para que possamos caracterizar essa definição de um modo mais palpável.

Proposição 2.14. Seja $\left(A_{n}\right)_{n \in \mathbb{N}}$ uma seqüência de conjuntos não-vazios em $C L(X)$, e suponha que $\operatorname{Li} A_{n} \neq \emptyset$. São equivalentes:

1. $p \in \operatorname{Li} A_{n}$;

2. Existe uma seqüência de pontos $\left(p_{n}\right)_{n \in \mathbb{N}}$, com $p_{n} \in A_{n}, \forall n \in \mathbb{N}$, tal que $p_{n} \rightarrow p$.

Demonstração. (1) $\Rightarrow(2)$ Tome $p \in \operatorname{Li} A_{n}$, e seja $S_{k}=B\left(p, \frac{1}{k}\right)$. Pela definição de limite inferior, existe $n_{k} \in \mathbb{N}$ tal que $n>n_{k} \Rightarrow S_{k} \cap A_{n} \neq \emptyset$. Podemos supor, sem perda de generalidade, que $n_{k+1}>n_{k}, \forall k \in \mathbb{N}$. Vamos construir a seqüência $\left(p_{n}\right)_{n \in \mathbb{N}}$.

- Se $n<n_{1}$, tome $p_{n} \in A_{n}$ (i.e., $p_{1} \in A_{1}, \cdots, p_{n_{1}-1} \in A_{n_{1}-1}$ );

- Se $n_{k} \leq n<n_{k+1}$, tome $p_{n} \in S_{k} \cap A_{n}$.

Dessa forma, e de acordo com o que assumimos, temos que, se $k \rightarrow \infty$, então $n_{k} \rightarrow \infty$. Além disso, se $n>n_{k}$, então $p_{n} \in S_{k}$ e, portanto:

$$
\left|p_{n}-p\right|<\frac{1}{k}
$$

Fazendo $k \rightarrow \infty$, temos $n \rightarrow \infty$ e, conseqüentemente, $p_{n} \rightarrow p$.

(2) $\Rightarrow$ (1) Se $p_{n} \rightarrow p$, então, para cada $V$ vizinhança de $p$, existe $n_{0} \in \mathbb{N}$ tal que $n>n_{0} \Rightarrow p_{n} \in$ $V$. Logo, $V \cap A_{n} \neq \emptyset, \forall n>n_{0}$, e $p \in \operatorname{Li} A_{n}$.

Proposição 2.15. Seja $\left(A_{n}\right)_{n \in \mathbb{N}}$ uma seqüência de conjuntos não-vazios em $C L(X)$, e suponha que Ls $A_{n} \neq \emptyset$. São equivalentes:

1. $p \in \operatorname{Ls} A_{n}$;

2. Existe uma seqüência de pontos $\left(p_{k_{n}}\right)_{n \in \mathbb{N}}, \operatorname{com} k_{1}<k_{2}<\cdots$ e $p_{k_{n}} \in A_{k_{n}}, \forall n \in \mathbb{N}$, tal que $p_{k_{n}} \rightarrow p$ 
Demonstração. Note que a demonstração desse fato é análoga à demonstração da proposição anterior, já que o fato de as vizinhanças não interceptarem todos os termos da seqüência a partir de um certo índice é contornado pela introdução de um subconjunto infinito arbitrário.

Um corolário imediato da proposição anterior (que nada mais é do que enunciar a implicação $(2) \Rightarrow(1)$ de outra maneira) é o seguinte: se $N \subseteq \mathbb{N}$ é um subconjunto infnito em $\mathbb{N}$ e $a_{n} \in A_{n}$, $\forall n \in N$, a convergência de $\left(a_{n}\right)_{n \in N}$ a $x \in X$ implica $x \in \operatorname{Ls} A_{n}$.

De posse dessas ferramentas, podemos obter uma caracterização da convergência no sentido de Kuratowski, de uma maneira mais adequada ao nosso contexto:

Proposição 2.16. Seja $\left(A_{n}\right)_{n \in \mathbb{N}}$ uma seqüência de conjuntos não-vazios em $C L(X)$. Então, $\left(A_{n}\right)_{n \in \mathbb{N}}$ converge no sentido de Kuratowski a um conjunto $A \in C L(X)$ se e somente se:

i) $\forall a \in A$ existe $\left(a_{n}\right)_{n \in \mathbb{N}}$, com $a_{n} \rightarrow a$ tal que, para cada $n \in \mathbb{N}, a_{n} \in A_{n}$;

ii) Sempre que $N \subseteq \mathbb{N}$ é um subconjunto infinito em $\mathbb{N}$ e $a_{n} \in A_{n}, \forall n \in N$, a convergência de $\left(a_{n}\right)_{n \in N}$ a $x \in X$ implica $x \in A$.

Demonstração. Basta usar as proposições 2.14 e 2.15 .

Vamos ver um exemplo de uma seqüência de conjuntos convergente no sentido de Kuratowski.

Exemplo 2.17. Seja $X=(0,2) \subseteq \mathbb{R}$, e seja $A_{n}=(0,1] \cup\left\{2-\frac{1}{n}\right\}, \forall n \in \mathbb{N}$. Vamos mostrar que $A_{n} \rightarrow A=(0,1]$ no sentido de Kuratowski.

i) Tome $x \in A$, e escolha a seqüência $\left(x_{n}\right)_{n \in \mathbb{N}}$ na qual $x_{n}=x, \forall n \in \mathbb{N}$. Temos, então, $x_{n} \in A_{n}$, $\forall n \in \mathbb{N}$, e $x_{n} \rightarrow x$, evidentemente.

ii) Seja $N \subseteq \mathbb{N}$ um subconjunto infinito em $\mathbb{N}$, com $x_{n} \in A_{n}, \forall n \in N$. Suponhamos que $x_{n} \rightarrow x \in X$, e vamos mostrar que $x \in A$. De fato, se $x \notin A$, então $x \in(1,2)$. Mas então $\exists n_{0} \in \mathbb{N}$ tal que, se $n \in N$ e $n>n_{0}$, então $x_{n} \in(1,2)$. Logo, $x_{n}=2-\frac{1}{n}$, e então $\left(x_{n}\right)_{n \in N}$ não converge em $X$, absurdo. Logo, $x \in A$.

\subsubsection{A topologia de Fell}

Nessa seção, apresentaremos uma topologia que, quando colocada sobre o espaço $C L(X)$, tornase compatível com a definição de convergência no sentido de Kuratowski. A topologia de Fell, também conhecida como topologia da convergência fechada, será denotada, ao longo desse texto, por $\tau_{C}$. A topologia $\tau_{C}$ é gerada pela seguinte base:

$$
\Gamma=\mathbb{A} \cup \mathbb{B}
$$


onde:

$\mathbb{A}=\left\{W_{1}(K): K\right.$ é compacto em $\left.X\right\}$, onde $W_{1}(K)=\{F \in C L(X): F \subseteq(X \backslash K)\}$

$\mathbb{B}=\left\{W_{2}(V): V\right.$ é aberto em $\left.X\right\}$, onde $W_{2}(V)=\{F \in C L(X): F \cap V \neq \emptyset\}$.

A proposição seguinte mostra que a convergência no sentido de Kuratowski de conjuntos fechados em um espaço métrico é compatível com a topologia $\tau_{C}$. Por fugir ao escopo desse trabalho, sua demonstração será omitida. Como referência, citamos o texto [1].

Proposição 2.18. Sejam $X$ um espaço métrico, $\left(A_{n}\right)_{n \in \mathbb{N}} \subseteq C L(X)$ uma seqüência de conjuntos não-vazios e $A \in C L(X)$ um conjunto não-vazio. Então:

$$
A=\mathrm{K}-\lim _{n \in \mathbb{N}} A_{n} \Leftrightarrow A_{n} \stackrel{\tau_{C}}{\longrightarrow} A .
$$

A próxima proposição estabelece uma relação entre a convergência no sentido de Wijsman e a convergência no sentido de Kuratowski:

Proposição 2.19. Sejam $\left(A_{n}\right)_{n \in \mathbb{N}} \subseteq C L(X)$ uma seqüência de conjuntos não-vazios e $A \in C L(X)$ um conjunto não-vazio. Se $\left(A_{n}\right)_{n \in \mathbb{N}}$ converge a $A$ no sentido de Wijsman, então também converge a $A$ no sentido de Kuratowski.

Demonstração. Tome $x \in A$. Como:

$$
d(\cdot, A)=\lim _{n \rightarrow \infty} d\left(\cdot, A_{n}\right)
$$

temos que:

$$
\lim _{n \rightarrow \infty} d\left(x, A_{n}\right)=0
$$

Logo, $x \in \operatorname{Li} A_{n}$, donde $A \subseteq \operatorname{Li} A_{n}$. Por outro lado, se $x \in \operatorname{Ls} A_{n}$, existe uma seqüência $\left(x_{k}\right)_{k \in \mathbb{N}}$, com $x_{k} \rightarrow x$, e uma seqüência crescente de inteiros $\left(n_{k}\right)_{k \in \mathbb{N}}$ tal que, para cada $k \in \mathbb{N}, x_{k} \in A_{n_{k}}$, pela proposição 2.15. Temos, então:

$$
\lim _{n \rightarrow \infty} d\left(x, A_{n_{k}}\right)=0 .
$$

Mas $d(\cdot, A)=\lim _{n \rightarrow \infty} d\left(\cdot, A_{n}\right)$, donde $d(x, A)=0$, e $x \in A$. Logo, Ls $A_{n} \subseteq A$, e segue que $A=\mathrm{K}-\lim _{n \in \mathbb{N}} A_{n}$.

O exemplo seguinte mostra que a convergência de conjuntos em $\tau_{C}$ não implica, necessariamente, a convergência pontual de suas funções distância, o que significa que, em geral, a convergência no sentido de Wijsman é mais forte que a convergência no sentido de Kuratowski. 
Exemplo 2.20. Seja $X=(0,2) \subseteq \mathbb{R}$, e seja $A_{n}=(0,1] \cup\left\{2-\frac{1}{n}\right\}, \forall n \in \mathbb{N}$. Já vimos que a seqüência $\left(A_{n}\right)_{n \in \mathbb{N}}$ converge, no sentido de Kuratowski, a $A=(0,1]$. Vamos mostrar que essa convergência não se dá no sentido de Wijsman. Tome $x=\frac{7}{4} \in X$. Temos:

$$
d\left(x, A_{n}\right)=2-\frac{7}{4}-\frac{1}{n}=\frac{1}{4}-\frac{1}{n} .
$$

Logo, $\lim _{n \rightarrow \infty} d\left(x, A_{n}\right)=\frac{1}{4}$. Mas:

$$
d(x, A)=\frac{7}{4}-1=\frac{3}{4} .
$$

Portanto, $\left(A_{n}\right)_{n \in \mathbb{N}}$ não converge a $A=(0,1]$ no sentido de Wijsman.

A proposição 2.19 e o exemplo 2.20 deixam claro que, se não assumirmos nenhuma hipótese adicional sobre o espaço $X$, além das que já assumimos, a convergência de uma seqüência de conjuntos no sentido de Wijsman é mais forte que sua convergência no sentido de Kuratowski.

Na próxima seção, apresentaremos uma classe de espaços na qual essas convergências são equivalentes.

\subsubsection{Espaços com nice closed balls}

Nessa seção, abordaremos uma classe de espaços na qual ocorre a equivalência entre a convergência de conjuntos no sentido de Wijsman e a convergência de conjuntos no sentido de Kuratowski. Os resultados aqui apresentados foram extraídos do texto [1].

Definição 2.21. Seja $X$ um espaço métrico. Dizemos que $X$ possui nice closed balls se, dada $B \subseteq X$ bola fechada e não-compacta, tem-se $B=X$.

Exemplo 2.22. Apresentamos três exemplos de espaços com nice closed balls:

a) Se $X$ é discreto (isto é, $d$ é a métrica zero-um), então $X$ possui nice closed balls.

De fato, seja $\left(x_{n}\right)_{n \in \mathbb{N}}$ uma seqüência em $X$ sem pontos de acumulação, e seja $p \in X$. Nesse caso, $\liminf _{n \rightarrow \infty} d\left(p, x_{n}\right)=1$; caso contrário, $p$ seria ponto de acumulação de $\left(x_{n}\right)_{n \in \mathbb{N}}$. Dado $x \in X$, tem-se $d(x, p) \leq 1$.

b) $\mathbb{R}^{m}$ possui nice closed balls.

Mais geralmente, qualquer espaço $X$ em que um conjunto fechado e limitado seja compacto possui nice closed balls. Vejamos: seja $\left(x_{n}\right)_{n \in \mathbb{N}}$ uma seqüência em $X$ sem pontos de acumulação. É claro que $\left(x_{n}\right)_{n \in \mathbb{N}}$ fica fora de cada bola de $X$, para $n$ suficientemente grande (caso contrário, haveria uma subseqüência de $\left(x_{n}\right)_{n \in \mathbb{N}}$ convergente). Assim, para cada $p \in X, \liminf _{n \rightarrow \infty} d\left(p, x_{n}\right)=+\infty$. 
c) Seja $X \subset l_{\infty}(\mathbb{K})$ o espaço métrico dado por:

$$
X=\left\{e_{n}: n \in \mathbb{N}\right\} \cup\left\{\frac{1}{n} e_{n}: n \in \mathbb{N}\right\} \cup\{0\},
$$

com $d$ a métrica induzida pela norma de $l_{\infty}(\mathbb{K})$. Então, $X$ possui nice closed balls.

De fato, para cada seqüência $\left(x_{n}\right)_{n \in \mathbb{N}} \subset X$ sem pontos de acumulação e para cada ponto $p \in X$, temos $d\left(p, x_{n}\right)=1$, para $n$ suficientemente grande (ou $p$ seria ponto de acumulação de $\left.\left(x_{n}\right)_{n \in \mathbb{N}}\right)$. Além disso, o diâmetro de $X$ é 1 . Dessa forma, para cada $x \in X$, temos $d(p, x) \leq 1=$ $\liminf _{n \rightarrow \infty} d\left(p, x_{n}\right)$.

O próximo lema nos fornece uma caracterização de tais espaços.

Lema 2.23. Seja $X$ um espaço métrico. São equivalentes:

1. Para cada $p \in X$ e cada $x \in X$, sempre que $\left(x_{n}\right)_{n \in \mathbb{N}}$ é uma seqüência em $X$ sem pontos de acumulação, tem-se:

$$
d(p, x) \leq \liminf _{n \rightarrow \infty} d\left(p, x_{n}\right)
$$

2. Se $B \subseteq X$ é uma bola fechada não-compacta, então $B=X$.

Demonstração. (1) $\Rightarrow(2)$ Seja $\overline{B(p, \epsilon)}$ uma bola fechada e não-compacta em $X$. Seja $\left(x_{n}\right)_{n \in \mathbb{N}}$ uma seqüência nessa bola sem nenhum ponto de acumulação, e seja $\alpha=\liminf _{n \rightarrow \infty} d\left(p, x_{n}\right)$. Por hipótese, $X \subseteq \overline{B(p, \alpha)}$. Mas, para cada $n \in \mathbb{N}, d\left(p, x_{n}\right) \leq \epsilon$. Logo, $\alpha \leq \epsilon$. Assim, $X=\overline{B(p, \epsilon)}$.

(2) $\Rightarrow(1)$ Fixe $p \in X$, e seja $\left(x_{n}\right)_{n \in \mathbb{N}}$ uma seqüência em $X$ sem pontos de acumulação. Se $\liminf _{n \rightarrow \infty} d\left(p, x_{n}\right)=+\infty$, então (1) está satisfeita. Suponhamos que $\liminf _{n \rightarrow \infty} d\left(p, x_{n}\right)=\alpha<+\infty$, e que, para algum $x \in X, d(p, x)>\alpha$. Tome:

$$
\epsilon=\frac{\alpha+d(p, x)}{2}
$$

Então, $\overline{B(p, \epsilon)}$ contém infinitos termos de $\left(x_{n}\right)_{n \in \mathbb{N}}$. Como $\left(x_{n}\right)_{n \in \mathbb{N}}$ não possui pontos de acumulação, $\overline{B(p, \epsilon)}$ não é compacto. Além disso, $\overline{B(p, \epsilon)} \neq X$, pois $x \notin \overline{B(p, \epsilon)}$, contradição.

A proposição a seguir apresenta a relação desejada:

Teorema 2.24. Sejam $X$ um espaço métrico e $\left(A_{n}\right)_{n \in \mathbb{N}} \subseteq C L(X)$ uma seqüência de conjuntos nâo-vazios. São equivalentes:

1. Se $\left(A_{n}\right)_{n \in \mathbb{N}}$ é convergente no sentido de Kuratowski a $A \in C L(X)$, então também o é no sentido de Wijsman; 
2. X possui nice closed balls.

Demonstração. $(1) \Rightarrow(2)$ Suponhamos que a convergência no sentido de Kuratowski implique a convergência no sentido de Wijsman, e vamos mostrar que $X$ possui nice closed balls. Suponha, por absurdo, que $X$ não possui nice closed balls. Então, podemos escolher pontos $p$ e $x$ em $X$ e uma seqüência $\left(x_{n}\right)_{n \in \mathbb{N}}$ em $X$ sem pontos de acumulação de modo que:

$$
d(p, x)>\liminf _{n \rightarrow \infty} d\left(p, x_{n}\right) .
$$

Podemos assumir - passando a uma subseqüência, se necessário - que, para algum $\epsilon>0$, e para cada $n \in \mathbb{N}, d\left(p, x_{n}\right)<d(p, x)-\epsilon$. Para cada $n \in \mathbb{N}$, defina $C_{n}=\left\{x, x_{n}\right\}$, e seja $C=\{x\}$. Claramente, temos:

$$
\operatorname{Li} C_{n}=\operatorname{Ls} C_{n}=C .
$$

Mas, como $d\left(p, x_{n}\right)<d(p, x)$, segue que:

$$
\limsup _{n \rightarrow \infty} d\left(p, C_{n}\right)=\limsup _{n \rightarrow \infty} d\left(p, x_{n}\right) \leq d(p, x)-\epsilon=d(p, C)-\epsilon .
$$

Logo, $\left(d\left(\cdot, C_{n}\right)\right)_{n \in \mathbb{N}}$ não converge pontualmente a $d(\cdot, C)$, contradição.

$(2) \Rightarrow(1)$ Seja $\left(A_{n}\right)_{n \in \mathbb{N}} \subseteq C L(X)$ uma seqüência convergente, no sentido de Kuratowski, a $A \in C L(X)$. Fixe $p \in X$. Como $A \subseteq$ Li $A_{n}$, é claro que, mesmo sem assumirmos nenhuma hipótese adicional sobre $X$, temos:

$$
\limsup _{n \rightarrow \infty} d\left(p, A_{n}\right) \leq d(p, A) .
$$
que:

Falta mostrarmos que $d(p, A) \leq \liminf _{n \rightarrow \infty} d\left(p, A_{n}\right)$. Para cada $n \in \mathbb{N}$, escolha $x_{n} \in A_{n}$ de modo

$$
d\left(p, x_{n}\right)<d\left(p, A_{n}\right)+\frac{1}{n}
$$

Dessa forma, existe uma subseqüência $\left(x_{n_{k}}\right)_{k \in \mathbb{N}}$ de $\left(x_{n}\right)_{n \in \mathbb{N}}$ para a qual

$$
\lim _{k \rightarrow \infty} d\left(p, x_{n_{k}}\right)=\liminf _{n \rightarrow \infty} d\left(p, A_{n}\right) .
$$

Se $\left(x_{n_{k}}\right)_{k \in \mathbb{N}}$ possui um ponto de acumulação $x$, então $x \in \operatorname{Ls} A_{n}$, e, pela continuidade da métrica, $d(p, A) \leq d(p, x)=\liminf _{n \rightarrow \infty} d\left(p, A_{n}\right)$.

Se não, para cada $x \in X$ :

$$
d(p, x) \leq \liminf _{n \rightarrow \infty} d\left(p, x_{n}\right)=\liminf _{n \rightarrow \infty} d\left(p, A_{n}\right) .
$$

Em particular, isso vale para cada $x \in A$, donde $d(p, A) \leq \liminf _{n \rightarrow \infty} d\left(p, A_{n}\right)$. Logo, $\left(d\left(\cdot, A_{n}\right)\right)_{n \in \mathbb{N}}$ converge pontualmente a $d(\cdot, A)$. 


\section{5 r-Convergência}

Nessa seção, vamos definir a r-convergência de conjuntos, e relacioná-la à convergência uniforme de funçòes em subconjuntos limitados de um espaço normado. Cabe ressaltar que a mesma definição pode ser feita em um espaço métrico qualquer. No entanto, para evitar o uso de resultados demasiadamente fora de nosso contexto, optamos por trabalhar em uma classe mais restrita.

Definição 2.25. Sejam $E$ um espaço normado, $\left(A_{n}\right)_{n \in \mathbb{N}}$ uma seqüência de conjuntos não-vazios em $C L(E)$ e $A \in C L(E)$. Dizemos que $\left(A_{n}\right)_{n \in \mathbb{N}}$ é r-convergente a $A$ se, para cada $r>0$ e cada $\epsilon>0$, existe $n_{0} \in \mathbb{N}$ tal que, se $n>n_{0}$, ocorrem:

i) $A \cap r B[0,1] \subseteq A_{n}+\epsilon B[0,1]$;

ii) $A_{n} \cap r B[0,1] \subseteq A+\epsilon B[0,1]$.

Nesse caso, escrevemos $A=\mathrm{r}-\lim _{n \in \mathbb{N}} A_{n}$.

Exemplo 2.26. A seguir, apresentamos dois exemplos de seqüências de conjuntos r-convergentes:

a) Para cada $n \in \mathbb{N}$, seja $A_{n}=B\left[0, \frac{1}{n}\right] \subseteq \mathbb{R}^{2}$, e seja $A=\{0\} \subseteq \mathbb{R}^{2}$. Vamos mostrar que $A=\mathrm{r}-\lim _{n \in \mathbb{N}} A_{n}$. De fato, se $r>0$ e $\epsilon>0$, temos, com $n>\frac{1}{\epsilon}$ :

1. $A \cap r B[0,1]=\{0\} \subseteq A_{n}+\epsilon B[0,1]$;

2. $A_{n} \subseteq B[0, \epsilon]$ e $A+\epsilon B[0,1]=\epsilon B[0,1] \Rightarrow A_{n} \cap r B[0,1] \subseteq A+\epsilon B[0,1]$.

b) Seja $a \in \mathbb{R}$, com $a>1$. Para cada $n \in \mathbb{N}$, considere $A_{n}=\left[-a-\frac{1}{n}, a+\frac{1}{n}\right]$, e seja $A=[-a, a]$. Vamos mostrar que $A=\mathrm{r}-\lim _{n \in \mathbb{N}} A_{n}$. Se $r>0$ e $\epsilon>0$, temos, com $n>\frac{1}{\epsilon}$ :

1. $A \cap r[-1,1] \subseteq A_{n}+\epsilon[-1,1], \forall n>\frac{1}{\epsilon}$;

2. $A_{n} \cap r[-1,1] \subseteq A+\epsilon[-1,1], \forall n>\frac{1}{\epsilon}$.

Apresentaremos mais alguns exemplos de seqüências r-convergentes ao longo do capítulo 3, uma vez que a relação obtida entre a r-convergência de conjuntos de nível e convergência em norma de funcionais lineares contínuos facilita sobremaneira a obtenção de tais exemplos em espaços normados quaisquer.

O nosso próximo objetivo é demonstrar um resultado que relacione a r-convergência de conjuntos à topologia da convergência uniforme das funçôes distância em subconjuntos limitados de E. 
Denotemos por $C_{b}(E, \mathbb{R})$ o conjunto das funções contínuas de $E$ a $\mathbb{R}$ que são limitadas em cada subconjunto limitado de $E$. É claro que $C_{b}(E, \mathbb{R}) \subseteq C(E, \mathbb{R})$.

Definamos a seguinte família de semi-normas em $C_{b}(E, \mathbb{R})$ : para cada $a \in E$ fixo e para cada $n \in \mathbb{N}$,

$$
p_{n}(f)=\sup _{\|x-a\| \leq n}|f(x)|
$$

Pode-se mostrar que essa família de semi-normas determina uma topologia em $C_{b}(E, \mathbb{R})$, denotada por $\tau_{\wp}$, com certas propriedades que a tornam bastante interessante. Por fugirem ao escopo desse trabalho, omitiremos os detalhes sobre essa topologia. Uma discussão detalhada sobre ela pode ser encontrada em [14].

Note-se que $\tau_{\wp}$ independe da escolha do ponto $a \in E$, uma vez que a escolha de outro ponto apenas altera o centro das bolas que estamos considerando. Além disso, a convergência com respeito a essa topologia significa convergência uniforme nos subconjuntos limitados de $E$.

De fato, ao tomarmos o supremo dos valores, garantimos a uniformidade da convergência. $\mathrm{E}$, como todo subconjunto limitado de $E$ deve estar contido em uma bola, a nomenclatura passa a fazer sentido.

Cabe, aqui, uma observação importante sobre o comportamento dessa topologia no espaço dual de um espaço normado $E$. Evidentemente, $E^{\prime} \subseteq C_{b}(E, \mathbb{R})$. Vamos investigar o que ocorre com $E^{\prime}$, quando visto como subespaço de $C_{b}(E, \mathbb{R})$, com a topologia induzida por $\tau_{\wp}$.

Como a topologia independe da escolha do ponto $a \in E$, podemos tomar, em particular, $a=0$. Temos, então,

$$
p_{1}(f)=\sup _{\|x-0\| \leq 1}|f(x)|=\|f\| .
$$

Analogamente, $p_{n}(f)=n\|f\|$. Dessa forma, a base de $\tau_{\wp}$ é a mesma base de vizinhanças da origem definida pela topologia da norma. Portanto, a topologia da convergência uniforme em subconjuntos limitados em $C_{b}(E, \mathbb{R})$ se reduz à topologia da norma, quando restrita a $E^{\prime}$.

Considere, agora, a função $\psi: C L(E) \longrightarrow C_{b}(E, \mathbb{R})$, dada por $\psi(A)=d(\cdot, A)$. A partir dessa função, é possível identificar $C L(E)$ com um subconjunto do espaço métrico $C_{b}(E, \mathbb{R})$. Chamaremos de topologia da convergência uniforme das funções distância em subconjuntos limitados de $E$ a topologia induzida por $\tau_{\wp}$ no subconjunto $\psi(C L(E)) \subseteq C_{b}(E, \mathbb{R})$.

A proposição seguinte relaciona essa topologia à r-convergência.

Proposição 2.27. Sejam $E$ um espaço normado, $\left(A_{n}\right)_{n \in \mathbb{N}}$ uma seqüência de conjuntos não-vazios em $C L(E)$ e $A \in C L(E)$ um conjunto não-vazio. São equivalentes:

1. $A=\mathrm{r}-\lim _{n \in \mathbb{N}} A_{n}$ 
2. A seqüência $\left(d\left(\cdot, A_{n}\right)\right)_{n \in \mathbb{N}}$ converge uniformemente a $d(\cdot, A)$ em subconjuntos limitados.

Demonstração. (1) $\Rightarrow(2)$ Seja $r>0$, e suponhamos que $A=\mathrm{r}-\lim _{n \in \mathbb{N}} A_{n}$. Por hipótese, existem $n_{0} \in \mathbb{N}$ e $r_{1}>0$ tais que $A \cap r_{1} B[0,1] \neq \emptyset$ e $A_{n} \cap r_{1} B[0,1] \neq \emptyset, \forall n>n_{0}$.

De fato, como $A \neq \emptyset$, existe $r_{1}>0$ tal que $A \cap r_{1} B[0,1] \neq \emptyset$. Agora, dado $\epsilon>0$, existe $N \in \mathbb{N}$ tal que $A \cap r_{1} B[0,1] \subseteq A_{n}+\epsilon B[0,1], \forall n>N$. Mas então:

$$
A \cap r_{1} B[0,1] \subseteq\left(A_{n} \cap r_{1} B[0,1]\right)+\epsilon B[0,1], \forall n>N
$$

Como $\epsilon$ é arbitrário, podemos fazer $\epsilon \rightarrow 0$, e obtemos $n_{0} \in \mathbb{N}$ tal que $n>n_{0} \Rightarrow A \cap r_{1} B[0,1] \subseteq$ $A_{n} \cap r_{1} B[0,1]$. Portanto, $A_{n} \cap r_{1} B[0,1] \neq \emptyset, \forall n>n_{0}$.

Assim, para cada $x \in r B[0,1]$ e cada $n>n_{0}$, temos $d(x, A) \leq r+r_{1}$ e $d\left(x, A_{n}\right) \leq r+r_{1}$. Dado $\epsilon>0$, tome:

$$
r_{2}>2 r+r_{1}+\frac{\epsilon}{2}
$$

Seja $n_{1} \in \mathbb{N}$ tal que, para todo $n>n_{1}$, tenhamos:

- $A \cap r_{2} B[0,1] \subseteq A_{n}+\frac{\epsilon}{2} B[0,1]$

- $A_{n} \cap r_{2} B[0,1] \subseteq A+\frac{\epsilon}{2} B[0,1]$.

Para cada $n>n_{1}$ e cada $x \in B[0,1]$, tome $b=b(x, n) \in A_{n}$ com $\|b-x\| \leq d\left(x, A_{n}\right)+\frac{\epsilon}{2}$. Pela escolha de $r_{2}$, temos:

$$
\|b\| \leq\|b-x\|+\|x\|<r+r+r_{1}+\frac{\epsilon}{2}<r_{2} .
$$

Como $A_{n} \cap r_{2} B[0,1] \subseteq A+\frac{\epsilon}{2} B[0,1]$, existe $a=a(x, n) \in A$ com:

$$
\|a-b\| \leq \frac{\epsilon}{2}
$$

pois $b \in A_{n} \cap r_{2} B[0,1]$. Assim, para cada $x \in r B[0,1]$ e cada $n>n_{1}$, temos:

$$
d(x, A) \leq\|x-a\| \leq\|x-b\|+\|b-a\|<d\left(x, A_{n}\right)+\frac{\epsilon}{2}+\frac{\epsilon}{2}=d\left(x, A_{n}\right)+\epsilon
$$

Dessa forma, $\left|d(x, A)-d\left(x, A_{n}\right)\right|<\epsilon$, e, se $n>n_{1}$ :

$$
\sup _{x \in r B[0,1]}\left|d(x, A)-d\left(x, A_{n}\right)\right| \leq \epsilon .
$$

Logo, $\left(d\left(\cdot, A_{n}\right)\right)_{n \in \mathbb{N}}$ converge a $d(\cdot, A)$ uniformemente em $r B[0,1]$.

(2) $\Rightarrow$ (1) Fixe $r>0$ e $\epsilon>0$. Por hipótese, existe $n_{0} \in \mathbb{N}$ tal que, se $n>n_{0}$ :

$$
\sup _{x \in r B[0,1]}\left|d\left(x, A_{n}\right)-d(x, A)\right|<\epsilon .
$$


Seja $x \in A \cap r B[0,1]$, e fixe $n>n_{0}$. Como $d(x, A)=0$, deve-se ter $d\left(x, A_{n}\right)<\epsilon$. Asim, para algum $a_{n} \in A_{n}$, tem-se $\left\|x-a_{n}\right\|<\epsilon$, donde:

$$
A \cap r B[0,1] \subseteq A_{n}+\epsilon B[0,1], \forall n>n_{0} .
$$

É possível mostrar, de forma análoga, que $A_{n} \cap r B[0,1] \subseteq A+\epsilon B[0,1], \forall n>n_{0}$. Logo, $A=\mathrm{r}-\lim _{n \in \mathbb{N}} A_{n}$.

\subsection{A Convergência de Mosco}

A convergência de conjuntos no sentido de Mosco é muito semelhante à convergência, no sentido de Kuratowski, de conjuntos convexos. No entanto, a convergência no sentido de Mosco pode ser descrita apenas em termos das topologias da norma e fraca, sem referências à distância definida no espaço.

Ao contrário do que ocorre com a convergência no sentido de Kuratowski, a convergência no sentido de Mosco não faz sentido em espaços métricos em geral, uma vez que envolve a estrutura vetorial do espaço em questão. Cabe ressaltar que o foco do estudo da convergência no sentido de Mosco se dá em subconjuntos convexos de espaços reflexivos.

Assim como fizemos com a definição de convergência no sentido de Kuratowski, inicialmente vamos definir a convergência no sentido de Mosco da maneira como foi concebida, em [13]. Apesar disso, vamos trabalhar com uma caracterização mais próxima de nosso contexto.

Vamos definir o limite inferior forte e o limite superior fraco de uma seqüência de conjuntos:

Definição 2.28. Seja $E$ um espaço normado, e seja $\left(A_{n}\right)_{n \in \mathbb{N}}$ uma seqüência de conjuntos convexos e não-vazios em $C L(E)$. O limite inferior forte de $\left(A_{n}\right)_{n \in \mathbb{N}}$ é:

$$
\underline{\mathrm{s}}-\lim _{n \in \mathbb{N}} A_{n}=\left\{x \in E: x=\mathrm{s}-\lim _{n \rightarrow \infty} x_{n}, \operatorname{com} x_{n} \in A_{n}, \forall n \in \mathbb{N}\right\} .
$$

O limite superior fraco de $\left(A_{n}\right)_{n \in \mathbb{N}}$ é:

$$
\overline{\mathrm{w}}-\lim _{n \in \mathbb{N}} A_{n}=\left\{x \in E: x=\mathrm{w}-\lim _{n \rightarrow \infty} x_{k_{n}}, \operatorname{com} x_{k_{n}} \in A_{k_{n}}, \forall n \in \mathbb{N}\right\} .
$$

A partir dessas definições, podemos estabelecer a convergência no sentido de Mosco:

Definição 2.29. Seja $E$ um espaço normado, e seja $\left(A_{n}\right)_{n \in \mathbb{N}}$ uma seqüência de conjuntos convexos e não-vazios em $C L(E)$. Dizemos que $\left(A_{n}\right)_{n \in \mathbb{N}}$ converge no sentido de Mosco a um conjunto $A \subseteq E$ se:

$$
A=\underline{\mathrm{s}}-\lim _{n \in \mathbb{N}} A_{n}=\overline{\mathrm{w}}-\lim _{n \in \mathbb{N}} A_{n}
$$


Nesse caso, escrevemos $A=\mathrm{M}-\lim _{n \in \mathbb{N}} A_{n}$.

A proposição seguinte garante que, nesse caso, o conjunto $A$ é convexo e fechado:

Proposição 2.30. Seja $E$ um espaço normado, e seja $\left(A_{n}\right)_{n \in \mathbb{N}}$ uma seqüência de conjuntos convexos e não-vazios em $C L(E)$ que converge, no sentido de Mosco, a um conjunto $A \subseteq E$. Então, $A$ é convexo e fechado em $E$.

Demonstração. A convexidade de $A$ é evidente, a partir da convexidade de cada $A_{n}$. Vamos mostrar que $A \in C L(E)$. Por hipótese, $A=\underline{\mathrm{s}}-\lim _{n \in \mathbb{N}} A_{n}$.

Considere $a \in \bar{A}$. Então, existe uma seqüência $\left(a_{n}\right)_{n \in \mathbb{N}} \in A$ tal que $a_{n} \rightarrow a$. Como $a_{n} \in A, \forall n \in$ $\mathbb{N}$, segue que $a_{n}=\mathrm{s}-\lim _{k \rightarrow \infty} x_{k}^{(n)}, \operatorname{com} x_{k}^{(n)} \in A_{k}, \forall k \in \mathbb{N}$ suficientemente grande.

Para cada $n \in \mathbb{N}$, defina $y_{n}=x_{k}^{(n)}, \operatorname{com} x_{k}^{(n)} \in A_{k}$ e $k>n$. Observe que:

$$
\left\|a-y_{n}\right\|=\left\|a-a_{n}+a_{n}-y_{n}\right\| \leq\left\|a-a_{n}\right\|+\left\|a_{n}-x_{k}^{(n)}\right\| \stackrel{n \rightarrow \infty}{\longrightarrow} 0 .
$$

Logo, $a \in \underline{\mathbf{s}}-\lim _{n \in \mathbb{N}} A_{n}=A$, donde $A$ é fechado.

A seguir, vamos reescrever essa definição de uma maneira mais apropriada ao nosso contexto. A partir desse ponto, entenderemos a convergência no sentido de Mosco da seguinte forma:

Proposição 2.31. Seja $E$ um espaço normado, e seja $\left(A_{n}\right)_{n \in \mathbb{N}}$ uma seqüência de conjuntos convexos e não-vazios em $C L(E)$. Então, $\left(A_{n}\right)_{n \in \mathbb{N}}$ converge no sentido de Mosco a um conjunto convexo $A \in C L(E)$ se e somente se:

i) $\forall a \in A, \exists\left(a_{n}\right)_{n \in \mathbb{N}}$, com $a=\mathrm{s}-\lim _{n \rightarrow \infty} a_{n}$, tal que, para cada $n \in \mathbb{N}, a_{n} \in A_{n}$;

ii) Sempre que $N \subseteq \mathbb{N}$ é um subconjunto infinito em $\mathbb{N}$ e $a_{n} \in A_{n}, \forall n \in N$, a convergência fraca de $\left(a_{n}\right)_{n \in N}$ a $x \in X$ implica $x \in A$.

Demonstração. Análoga à proposição 2.16.

A seguir, vamos exemplificar uma seqüência que converge no sentido de Mosco:

Exemplo 2.32. Considere, em $\mathbb{R}$, os seguintes conjuntos fechados e convexos:

$$
A_{n}=\left[-\sum_{i=0}^{n} \frac{1}{2^{i}}, \sum_{i=0}^{n} \frac{1}{2^{i}}\right], \forall n \in \mathbb{N} \quad \text { e } \quad A=[-2,2] .
$$

Vamos mostrar que $A=\mathrm{M}-\lim _{n \in \mathbb{N}} A_{n}$. 
i) Tome $a \in A$. Se $a \in(-2,2)$, então existe $n_{0} \in \mathbb{N}$ tal que $n>n_{0} \Rightarrow a \in A_{n}$. Logo, nesse caso é fácil encontrar uma seqüência que satisfaça a primeira condição da proposição 2.31. Se $a=2$, tome $a_{n}=\sum_{i=0}^{n} \frac{1}{2^{2}}$. Temos que $a_{n} \in A_{n}, \forall n \in \mathbb{N}$, e $a_{n} \rightarrow a$, claramente. O mesmo raciocínio deve ser usado no caso $a=-2$.

ii) Seja $N \subset \mathbb{N}$ infinito, com $a_{n} \in A_{n}, \forall n \in N$. Seja $x \in \mathbb{R}$ tal que $x=\mathrm{w}-\lim _{n \rightarrow \infty} a_{n}$. Vamos mostrar que $x \in A$. Se $x \notin A$, então $|x|>2$. Como $x=\mathrm{w}-\lim _{n \rightarrow \infty} a_{n}$, deve-se ter, em particular, $i d(x)=\lim _{n \in N} i d\left(a_{n}\right)$, donde $x=\lim _{n \in N} a_{n}$, absurdo, pois $a_{n} \in[-2,2], \forall n \in N$. Logo, $x \in A$.

É fácil ver, a partir de sua definição, que a convergência no sentido de Mosco de uma seqüência de conjuntos implica a convergência, no sentido de Kuratowski, dessa mesma seqüência. Além disso, ambas as definições coincidem quando o espaço $E$ tem dimensão finita, uma vez que, nesse caso, as noções de convergência de seqüências de vetores utilizadas são equivalentes.

Denotemos por $C c(E)$ o conjunto dos subconjuntos fechados e convexos de $E$. A seguir, apresentaremos uma topologia em $C c(E)$ compatível com a convergência no sentido de Mosco, para o caso em que $E$ é completo.

Definição 2.33. Seja $E$ um espaço de Banach. A topologia de $M o s c o$ em $C c(E), \tau_{M}$, é a topologia gerada pelos conjuntos da forma:

$$
\{\{C \in C c(E): C \cap V \neq \emptyset\}: V \in O(E)\} \cup\{\{C \in C c(E): C \subseteq(E \backslash K)\}: K \in K(E)\}
$$

onde:

$O(E)$ : subconjuntos de $E$ não-vazios e abertos (na topologia da norma) em $E$;

$K(E)$ : subconjuntos de $E$ não-vazios e fracamente compactos em $E$.

A proposição seguinte apresenta a compatibilidade desejada:

Proposição 2.34. Sejam $E$ um espaço de Banach, $\left(A_{n}\right)_{n \in \mathbb{N}}$ uma seqüência de conjuntos não-vazios em $C c(E)$ e $A \in C c(E) \backslash\{\emptyset\}$. Então:

$$
A=\mathrm{M}-\lim _{n \in \mathbb{N}} A_{n} \Leftrightarrow A_{n} \stackrel{\tau_{M}}{\longrightarrow} A
$$

Por envolver uma parte da teoria que foge ao contexto dessa dissertação, optamos por omitir a demonstração desse resultado. Como referência, mencionamos o texto [2].

O próximo teorema estabelece uma relação entre as noções de convergência estudadas. A relação entre convergência no sentido de Mosco e convergência no sentido de Kuratowski já foi apresentada.

Teorema 2.35. Sejam $E$ um espaço reflexivo, $\left(A_{n}\right)_{n \in \mathbb{N}}$ uma seqüência de conjuntos não-vazios em $C c(E)$ e $A \in C c(E) \backslash\{\emptyset\}$. Verificam-se as seguintes condições: 
1. Se $A=\mathrm{r}-\lim _{n \in \mathbb{N}} A_{n}$, então $A=\mathrm{M}-\lim _{n \in \mathbb{N}} A_{n}$;

2. Se $A=\mathrm{M}-\lim _{n \in \mathbb{N}} A_{n}$, então $A=\mathrm{W}-\lim _{n \in \mathbb{N}} A_{n}$;

3. Se $A=\mathrm{W}-\lim _{n \in \mathbb{N}} A_{n}$, então $A=\mathrm{K}-\lim _{n \in \mathbb{N}} A_{n}$;

4. Se $\operatorname{dim} E<+\infty$, todas as noções de convergência são equivalentes.

Demonstração. (1) Suponhamos que $A=\mathrm{r}-\lim _{n \in \mathbb{N}} A_{n}, \forall r>r_{0}>0$. Vamos, primeiramente, mostrar que $A \subseteq \underline{\mathrm{s}}-\lim _{n \in \mathbb{N}} A_{n}$. Tome $x \in A$, e seja $r>\max \left\{r_{0},\|x\|\right\}$. Para qualquer $\epsilon>0$, temos, pela primeira condição da definição de r-convergência, que $x \in A_{n}+\epsilon B[0,1]$, para $n$ suficientemente grande. Logo, $d\left(x, A_{n}\right) \leq \epsilon$.

Com esse raciocínio, temos que $d\left(x, A_{n}\right) \rightarrow 0$, quando $n \rightarrow \infty$. Logo, $x \in \underline{\mathrm{s}}-\lim _{n \in \mathbb{N}} A_{n}$, donde segue que $A \subseteq \underline{s}-\lim _{n \in \mathbb{N}} A_{n}$.

Mostremos, agora, que $\overline{\mathrm{w}}-\lim _{n \in \mathbb{N}} A_{n} \subseteq A$. Sejam $x \in E$ e $x_{k} \in A_{n_{k}}$, com $\left(A_{n_{k}}\right)_{k \in \mathbb{N}}$ uma subseqüência de $\left(A_{n}\right)_{n \in \mathbb{N}}$. Suponhamos que $x=\mathrm{w}-\lim _{n \rightarrow \infty} x_{n}$, e vamos mostrar que $x \in A$.

Como $x=\mathrm{w}-\lim _{n \rightarrow \infty} x_{n}$, existe $r>r_{0}>0$ tal que $\left\|x_{k}\right\| \leq r, \forall k \in \mathbb{N}$. Assim, $x_{k} \in A_{k} \cap$ $r B[0,1], \forall k \in \mathbb{N}$. Pela segunda condição da definição de r-convergência, tem-se $x_{k} \in A+\epsilon B[0,1]$, para $k$ suficientemente grande. Como $A+\epsilon B[0,1]$ é fechado e convexo, segue que $x \in A+\epsilon B[0,1]$. Mas $\epsilon$ é arbitrário, donde segue que $x \in A$.

Finalmente, concluímos que $A=\mathrm{M}-\lim _{n \in \mathbb{N}} A_{n}$.

(2) A demonstração dessa implicação envolve algumas estruturas topológicas que fogem ao contexto dessa dissertação. Como referência, podemos citar [2].

(3) Segue da proposição 2.19.

(4) Vamos mostrar a recíproca de (1). Suponhamos que $A=\mathrm{M}-\lim _{n \in \mathbb{N}} A_{n}$ e que $\operatorname{dim} E<+\infty$. Vamos mostrar que $A=\mathrm{r}-\lim _{n \in \mathbb{N}} A_{n}, \forall r>r_{0}>0$.

Suponhamos que $A \cap r B[0,1] \neq \emptyset, \forall r>r_{1}$. Como $A \cap r B[0,1]$ é compacto (já que é fechado e limitado), pode-se afirmar que $\forall \epsilon>0$ existem $x_{1}, \cdots, x_{n} \in A \cap r B[0,1]$ tais que:

$$
A \cap r B[0,1] \subseteq \bigcup_{i=1}^{n}\left(\left\{x_{i}\right\}+\frac{\epsilon}{2} B[0,1]\right)
$$

Como $A=\mathrm{M}-\lim _{n \in \mathbb{N}} A_{n}$, existe $n_{\epsilon} \in \mathbb{N}$ tal que, se $n>n_{\epsilon}, d\left(x_{i}, A_{n}\right) \leq \frac{\epsilon}{2}$, para $i=1, \cdots, n$. Logo:

$$
\left\{x_{i}\right\}+\frac{\epsilon}{2} B[0,1] \subseteq A_{n}+\epsilon B[0,1]
$$


donde $A \cap r B[0,1] \subseteq A_{n}+\epsilon B[0,1]$, e a primeira condição da definição de r-convergência está satisfeita.

Agora, suponhamos $A_{n} \cap r B[0,1] \neq \emptyset, \forall r>r_{2}>0, \forall n>n_{2}>0$. Vamos admitir, por absurdo, que existem $\bar{r}>r_{2}$ e $\bar{\epsilon}>0$ tais que:

$$
A_{n_{k}} \cap \bar{r} B[0,1] \nsubseteq A+\bar{\epsilon} B[0,1]
$$

com $\left(A_{n_{k}}\right)_{k \in \mathbb{N}}$ uma subseqüência de $\left(A_{n}\right)_{n \in \mathbb{N}}$. Então, existe uma seqüência $\left(x_{k}\right)_{k \in \mathbb{N}}$, com $x_{k} \in$ $A_{n_{k}} \cap \bar{r} B[0,1], \forall k \in \mathbb{N}$ e $x_{k} \notin A+\bar{\epsilon} B[0,1], \forall k \in \mathbb{N}$ que é limitada em $E$.

Logo, $\left(x_{k}\right)_{k \in \mathbb{N}}$ possui uma subseqüência $\left(x_{k_{l}}\right)_{l \in \mathbb{N}}$ tal que $x_{k_{l}} \rightarrow x \in E$. Como $\overline{\mathrm{w}}-\lim _{n \in \mathbb{N}} A_{n} \subseteq A$, devemos ter $x \in A$. Mas $x \notin A+\epsilon B[0,1], \forall \epsilon<\bar{\epsilon}$, contradição.

Assim, deve ser válida a segunda condição da definição de r-convergência e, portanto, temos $A=\mathrm{r}-\lim _{n \in \mathbb{N}} A_{n}$.

Com relação aos demais itens, temos que, se $\operatorname{dim} E<+\infty$, então $E$ é isomorfo a $\mathbb{R}^{n}$, para algum $n \in \mathbb{N}$, donde a convergência no sentido de Wijsman é equivalente à convergência no sentido de Kuratowski. Como as convergências nos sentidos de Kuratowski e de Mosco em espaços de dimensão finita também são equivalentes, seguem as recíprocas dos itens (2) e (3).

As recíprocas das três primeiras afirmações acima são falsas. Um exemplo disso, no caso (2), será apresentado no capítulo dedicado às relações entre convergência de conjuntos e convergência de funcionais lineares contínuos. No caso (3), já vimos um contra-exemplo, na seção sobre convergência no sentido de Kuratowski.

Finalmente, para o caso (1), consideremos o seguinte exemplo:

Exemplo 2.36. Vamos mostrar que a r-convergência de uma seqüência de conjuntos pode ser estritamente mais forte que sua convergência no sentido de Mosco.

Considere, em $l_{2}(\mathbb{R})$, os seguintes conjuntos:

$$
\begin{gathered}
S=B \cap\left\{x=\left(x^{(1)}, x^{(2)}, \cdots\right) \in l_{2}(\mathbb{R}): 0 \leq x^{(k)} \leq 1, \forall k \in \mathbb{N}\right\} \mathrm{e} \\
S_{n}=B \cap\left\{x=\left(x^{(1)}, x^{(2)}, \cdots\right) \in l_{2}(\mathbb{R}): 0 \leq x^{(k)} \leq 1+n^{-\alpha} k, \forall k \in \mathbb{N}\right\},
\end{gathered}
$$

$\operatorname{com} B=\left\{x \in l_{2}:\|x\| \leq 2\right\}$ e $\alpha>0$ dado. Vamos mostrar que $S=\mathrm{M}-\lim _{n \in \mathbb{N}} S_{n}$ :

i) Observe que $S \subseteq S_{n}, \forall n \in \mathbb{N}$. Logo, se $x \in S$, podemos tomar $x_{n}=x, \forall n \in \mathbb{N}$, e temos que $x_{n} \in S_{n}, \forall n \in \mathbb{N}$, e $x=\mathrm{s}-\lim _{n \rightarrow \infty} x_{n}$. 
ii) Seja $N \subseteq \mathbb{N}$ infinito, e seja $x_{n} \in S_{n}, \forall n \in N$. Suponhamos que exista $x \in l_{2}(\mathbb{R})$ tal que $x=\mathrm{w}-\lim _{n \rightarrow \infty} x_{n}$. Vamos mostrar que $x \in S$.

Se $x \notin S$, existe $k \in \mathbb{N}$ tal que $x^{(k)}>1$. Escrevamos $x^{(k)}=1+\epsilon$, com $\epsilon>0$. Seja $\pi_{k}$ a k-ésima projeção em $l_{2}(\mathbb{R})$. Como $x=\mathrm{w}-\lim _{n \rightarrow \infty} x_{n}$, temos, em particular, que $\pi_{k}(x)=\lim _{n \in N} \pi_{k}\left(x_{n}\right)$. Logo, $x^{(k)}=\lim _{n \in N} x_{n}^{(k)}$. Mas, por definição, temos $0 \leq x_{n}^{(k)} \leq 1+n^{-\alpha} k$. Assim, se escolhermos $n$ suficientemente grande, temos $x_{n}^{(k)}<1+\epsilon$, e $x^{(k)} \neq \lim _{n \in N} x_{n}^{(k)}$, absurdo.

No entanto, $S \neq \mathrm{r}-\lim _{n \in \mathbb{N}} S_{n}$. Vamos mostrar que não é verdade que, dado qualquer $\epsilon>0$, temos $S_{n} \subseteq S+\epsilon B[0,1]$ para todo $n$ suficientemente grande. Observe que:

$$
S+\epsilon B[0,1] \subseteq B \cap\left\{x \in l_{2}(\mathbb{R}): 0 \leq x^{(k)} \leq 1+\epsilon, \forall k \in \mathbb{N}\right\}
$$

Logo, não é possível garantir, apenas impondo restrições para o valor de $n$, que se tenha $S_{n} \subseteq$ $S+\epsilon B[0,1]$.

Finalmente, a proposição seguinte apresenta um fato sobre r-convergência e convergência de Mosco que será utilizado ao longo do capítulo sobre convergência de funcionais lineares contínuos.

Proposição 2.37. Sejam $\left(A_{n}\right)_{n \in \mathbb{N}}$ uma seqüência de conjuntos convexos e fechados em um espaço normado $E, A$ um conjunto convexo e fechado em $E$ e $\left(x_{n}\right)_{n \in \mathbb{N}} \subseteq E$ tal que $x_{n} \stackrel{\|\cdot\|}{\longrightarrow} x \in E$. Então:

1. Se $A=\mathrm{M}-\lim _{n \in \mathbb{N}} A_{n}$, então $x+A=\mathrm{M}-\lim _{n \in \mathbb{N}}\left(x_{n}+A_{n}\right)$;

2. Se $A=\mathrm{r}-\lim _{n \in \mathbb{N}} A_{n}$, então $x+A=\mathrm{r}-\lim _{n \in \mathbb{N}}\left(x_{n}+A_{n}\right)$;

Demonstração. Definamos:

$$
B_{n}=x_{n}+A_{n}=\left\{x_{n}+a: a \in A_{n}\right\} \quad \text { e } \quad B=x+A=\{x+a: a \in A\} .
$$

(1) Inicialmente, precisamos mostrar que, para cada $b \in B$, existe uma seqüência $\left(b_{n}\right)_{n \in \mathbb{N}}$ tal que $b=\mathrm{s}-\lim _{n \rightarrow \infty} b_{n}$ e $b_{n} \in B_{n}, \forall n \in \mathbb{N}$.

Tome $b \in B$. Então, $b=x+a$, para algum $a \in A$. Por hipótese, existe $\left(a_{n}\right)_{n \in \mathbb{N}}$, com $a_{n} \in A_{n}$, $\forall n \in \mathbb{N}$, tal que $a=\mathrm{s}-\lim _{n \rightarrow \infty} a_{n}$. Mas $x=\mathrm{s}-\lim _{n \rightarrow \infty} x_{n}$. Logo, se tomarmos $b_{n}=x_{n}+a_{n}$, temos $b=\mathrm{s}-\lim _{n \rightarrow \infty} b_{n}$ e $b_{n} \in B_{n}, \forall n \in \mathbb{N}$.

Devemos mostrar, agora, que, se $N \subseteq \mathbb{N}$ é infinito e $b_{n} \in B_{n}, \forall n \in N$, a convergência fraca de $\left(b_{n}\right)_{n \in N}$ a $b \in X$ implica que $b \in B$. Sabemos que, se $x=\mathrm{s}-\lim _{n \rightarrow \infty} x_{n}$, então $x=\mathrm{w}-\lim _{n \rightarrow \infty} x_{n}$. Tome $N \subseteq \mathbb{N}$ infinito, e seja $b_{n} \in B_{n}, \forall n \in N, \operatorname{com} b=\mathrm{w}-\lim _{n \rightarrow \infty} b_{n}$. Mas $b_{n}=x_{n}+a_{n}, \operatorname{com} a_{n} \in A_{n}$, $\forall n \in N$. Então:

$$
b=\mathrm{w}-\lim _{n \rightarrow \infty}\left(x_{n}+a_{n}\right)=\mathrm{w}-\lim _{n \rightarrow \infty} x_{n}+\mathrm{w}-\lim _{n \rightarrow \infty} a_{n}=x+a \in B,
$$


como queríamos.

(2) Sabemos que $A=\mathrm{r}-\lim _{n \in \mathbb{N}} A_{n}$, e precisamos mostrar que, para cada $r>0$ e cada $\epsilon>0$ existe $n_{0} \in \mathbb{N}$ tal que $n>n_{0}$ implica:

i) $B \cap r B[0,1] \subseteq B_{n}+\epsilon B[0,1]$;

ii) $B_{n} \cap r B[0,1] \subseteq B+\epsilon B[0,1]$.

Sejam $r>0$ e $\epsilon>0$. Por hipótese, existe $n_{0} \in \mathbb{N}$ tal que, se $n>n_{0}$ :

$$
A \cap r B[0,1] \subseteq A_{n}+\frac{\epsilon}{2} B[0,1]
$$

Então, se $n>n_{0}$, temos:

$$
(x+A) \cap r B[0,1] \subseteq\left(x+A_{n}\right)+\frac{\epsilon}{2} B[0,1]
$$

donde, se $n>n_{0}$ :

$$
B \cap r B[0,1] \subseteq\left(x+A_{n}\right)+\frac{\epsilon}{2} B[0,1]
$$

Mas, como $x_{n} \rightarrow x$ em norma, segue que existe $n_{1} \in \mathbb{N}$ tal que, se $n>n_{1}$ :

$$
x \in\left\{x_{n}\right\}+\frac{\epsilon}{2} B[0,1] .
$$

Logo, se $n>n_{1}$ :

$$
\left(x+A_{n}\right)+\frac{\epsilon}{2} B[0,1] \subseteq\left(x_{n}+A_{n}\right)+\epsilon B[0,1]=B_{n}+\epsilon B[0,1] .
$$

Tome $n_{2}>\max \left\{n_{0}, n_{1}\right\}$. Então, se $r>0, \epsilon>0$ e $n>n_{2}$, tem-se $B \cap r B[0,1] \subseteq B_{n}+\epsilon B[0,1]$. Analogamente, prova-se a outra asserção. 


\section{Capítulo 3}

\section{Convergência de conjuntos de nível de funcionais lineares contínuos}

Esse capítulo será dedicado ao estudo das relações existentes entre os diversos tipos de convergência de funcionais lineares contínuos e a convergência, em cada um dos sentidos já apresentados, de seus conjuntos de nível. Como base para esse capítulo, utilizaremos o texto científico [3].

Inicialmente, vamos definir o conjunto de nível de um funcional linear contínuo.

Definição 3.1. Sejam $E$ um espaço normado sobre $\mathbb{K}, f \in E^{\prime}$ e $\alpha \in \mathbb{K}$. O conjunto de nível $\alpha$ de $f$ é:

$$
L(f ; \alpha)=\{x \in E: f(x)=\alpha\}
$$

Cabe notar que, em função da continuidade dos funcionais lineares, seus conjuntos de nível são conjuntos fechados em seu domínio. Assim, $L(f ; \alpha) \in C L(E)$.

Exemplo 3.2. Apresentaremos alguns exemplos que ilustram essa definição:

a) Seja $f: E \longrightarrow \mathbb{K}$ um funcional linear contínuo. Sempre se pode escrever $E=\bigcup_{\alpha \in \mathbb{K}} L(f ; \alpha)$. Se $f: E \rightarrow \mathbb{K}$ é o funcional nulo, tem-se $L(f ; 0)=E$.

b) Considere $E=(C[0,1] ; \mathbb{R})$, e fixe $t \in[0,1]$. Seja $f: E \rightarrow \mathbb{R}$ dado por $f(x)=x(t)$. Então,

$$
L(f ; \alpha)=\{x \in(C[0,1] ; \mathbb{R}): f(x)=\alpha\}=\{x \in(C[0,1] ; \mathbb{R}): x(t)=\alpha\} .
$$

c) Considere, novamente, $E=(C[0,1] ; \mathbb{R})$. Seja $f: E \longrightarrow \mathbb{R}$ dada por:

$$
f(x)=\int_{0}^{1} x(t) d t
$$


Então:

$$
L(f ; \alpha)=\left\{x \in E: \int_{0}^{1} x(t) d t=\alpha\right\} .
$$

d) Considere $E=l_{2}(\mathbb{R})$, e fixe $\zeta=\left(\zeta_{n}\right)_{n \in \mathbb{N}} \in l_{2}(\mathbb{R})$. Seja $f: E \longrightarrow \mathbb{R}$ dada por:

$$
f(x)=\sum_{n=1}^{\infty} x_{n} \zeta_{n} .
$$

Nesse caso, $L(f ; \alpha)=\left\{x \in l_{2}(\mathbb{R}): \sum_{n=1}^{\infty} x_{n} \zeta_{n}=\alpha\right\}$.

Observação 3.3. Se $x_{0} \in E$ é tal que $f\left(x_{0}\right)=\alpha$, então $L(f ; \alpha)=x_{0}+L(f ; 0)$. De fato, se $x \in L(f ; \alpha)$ e $x \neq x_{0}$, existe $v \in E$ tal que $x=x_{0}+v$. Então:

$$
\alpha=f(x)=f\left(x_{0}\right)+f(v)=\alpha+f(v) \Rightarrow f(v)=0 .
$$

Logo, $x \in x_{0}+L(f ; 0)$. Reciprocamente, se $x \in x_{0}+L(f ; 0)$, então $x=x_{0}+v, \operatorname{com} f(v)=0$, donde $f(x)=f\left(x_{0}\right)=\alpha$.

Deste ponto em diante, apresentaremos os resultados que relacionam convergência de funcionais lineares contínuos e convergência de seus conjuntos de nível. Até o final desse capítulo, a menos de menção explícita em contrário, $E$ denotará um espaço normado sobre $\mathbb{R}$.

\subsection{Convergência Fraca- $\star$}

O teorema seguinte é o principal resultado que relaciona a convergência fraca- $\star$ de uma seqüência de funcionais lineares contínuos à convergência, no sentido de Kuratowski, dos conjuntos de nível dessa seqüência de funcionais.

Teorema 3.4. Sejam $E$ um espaço normado, $\left(f_{n}\right)_{n \in \mathbb{N}}$ uma seqüência de funcionais não-nulos em $E^{\prime}$ e $f \in E^{\prime} \backslash\{0\}$. São equivalentes:

1. Para cada $\alpha \in \mathbb{R}, L(f ; \alpha)=\mathrm{K}-\lim _{n \in \mathbb{N}} L\left(f_{n} ; \alpha\right)$;

2. Para cada $N \subseteq \mathbb{N}$ infinito, as condições $x=\mathrm{s}-\lim _{n \in N} x_{n}$ e $\alpha=\lim _{n \in N} f_{n}\left(x_{n}\right)$ implicam $f(x)=\alpha$;

3. A seqüência $\left(f_{n}\right)_{n \in \mathbb{N}}$ é limitada, e $f=\mathrm{w}^{\star}-\lim _{n \rightarrow \infty} f_{n}$;

4. Sempre que $x=\mathrm{s}-\lim _{n \rightarrow \infty} x_{n}, f(x)=\lim _{n \rightarrow \infty} f_{n}\left(x_{n}\right)$. 
Demonstração. (1) $\Rightarrow$ (2) Suponhamos $x=s-\lim _{n \in N} x_{n}$ e $\alpha=\lim _{n \in N} f_{n}\left(x_{n}\right)$. Como $f \neq 0, f$ é sobrejetor. Portanto, podemos escolher $z \in E$ tal que $f(z)=1-\alpha$.

Escolha, por (1), uma seqüência $\left(z_{n}\right)_{n \in \mathbb{N}}$ tal que $z=\mathrm{s}-\lim _{n \rightarrow \infty} z_{n}$ e, para cada $n \in \mathbb{N}, z_{n} \in$ $L\left(f_{n}, 1-\alpha\right)$. É claro que:

$$
\lim _{n \in N} f_{n}\left(x_{n}+z_{n}\right)=\lim _{n \in N} f_{n}\left(x_{n}\right)+\lim _{n \in N} f_{n}\left(z_{n}\right)=\alpha+1-\alpha=1 .
$$

Se colocarmos $\alpha_{n}=f_{n}\left(x_{n}+z_{n}\right)$, então existe $n_{0} \in N$ tal que, se $n>n_{0}$ e $n \in N$, então

$$
\frac{x_{n}+z_{n}}{\alpha_{n}} \in L\left(f_{n} ; 1\right) \quad \text { e } \quad x+z=\mathrm{s}-\lim _{n>n_{0}}\left(x_{n}+z_{n}\right) .
$$

Por $(1), x+z \in L(f ; 1)$, donde $f(x)=1-f(z)=1-1+\alpha=\alpha$.

(2) $\Rightarrow$ (3) Suponha, por absurdo, que a seqüência $\left(f_{n}\right)_{n \in \mathbb{N}}$ não seja limitada. Seja $N \subseteq \mathbb{N}$ um subconjunto infinito em $\mathbb{N}$ tal que, para cada $n \in N,\left\|f_{n}\right\|>n$. Para cada $n \in N$, existe $x_{n} \in E$ $\operatorname{com}\left\|x_{n}\right\|<\frac{1}{n}$ e $f_{n}\left(x_{n}\right)=1$.

É claro que $x_{n} \stackrel{\|\cdot\|_{0}}{\longrightarrow} 0$. Como $f(0)=0$ e $\lim _{n \in N} f_{n}\left(x_{n}\right)=1$, obtemos uma contradição. Logo, $\left(f_{n}\right)_{n \in \mathbb{N}}$ deve ser limitada.

Agora, fixe $x \in E$. Como $\left(f_{n}\right)_{n \in \mathbb{N}}$ é limitada, tanto $\liminf _{n \rightarrow \infty} f_{n}(x)$ como $\limsup _{n \rightarrow \infty} f_{n}(x)$ existem e são finitos. Mas então:

- $\limsup _{n \rightarrow \infty} f_{n}(x)=\lambda_{1} \Rightarrow \exists N_{1} \subseteq \mathbb{N}$ infinito tal que $f_{n}(x) \rightarrow \lambda_{1}$, com $n \in N_{1}$;

- $\liminf _{n \rightarrow \infty} f_{n}(x)=\lambda_{2} \Rightarrow \exists N_{2} \subseteq \mathbb{N}$ infinito tal que $f_{n}(x) \rightarrow \lambda_{2}$, com $n \in N_{2}$.

Aplicando (2) para $x_{n}=x, \forall n \in \mathbb{N}$, temos:

$$
\begin{gathered}
x=\mathrm{s}-\lim _{n \rightarrow \infty} x \mathrm{e} \lim _{n \in N_{1}} f_{n}(x)=\lambda_{1} \Rightarrow f(x)=\lambda_{1} \mathrm{e} \\
x=\mathrm{s}-\lim _{n \rightarrow \infty} x \mathrm{e} \lim _{n \in N_{2}} f_{n}(x)=\lambda_{2} \Rightarrow f(x)=\lambda_{2} .
\end{gathered}
$$

Logo, $\liminf _{n \rightarrow \infty} f_{n}(x)=\limsup _{n \rightarrow \infty} f_{n}(x)$, donde $\lim _{n \rightarrow \infty} f_{n}(x)$ existe e $f(x)=\lim _{n \rightarrow \infty} f_{n}(x)$.

$(3) \Rightarrow(4)$ Observe que:

$$
\left|f_{n}\left(x_{n}\right)-f(x)\right| \leq\left|f_{n}\left(x_{n}\right)-f_{n}(x)\right|+\left|f_{n}(x)-f(x)\right| \leq\left\|f_{n}\right\|\left\|x_{n}-x\right\|+\left|f_{n}(x)-f(x)\right| .
$$

Por (3), $\left(f_{n}\right)_{n \in \mathbb{N}}$ é limitada e $f(x)=\lim _{n \rightarrow \infty} f_{n}(x)$. Se $x=\mathrm{s}-\lim _{n \rightarrow \infty} x_{n}$, então $f(x)=\lim _{n \rightarrow \infty} f_{n}\left(x_{n}\right)$. 
$(4) \Rightarrow(1)$ Fixe $\alpha \in \mathbb{R}$. Queremos mostrar que $L(f ; \alpha)=\mathrm{K}-\lim _{n \in \mathbb{N}} L\left(f_{n} ; \alpha\right)$. Por (4), a condição ii) da proposição 2.16 é satisfeita, uma vez que, se $x=\mathrm{s}-\lim _{n \rightarrow \infty} x_{n}$, então $f_{n}\left(x_{n}\right) \rightarrow f(x)$, isto é, se $x_{n} \in L\left(f_{n} ; \alpha\right)$ e $x=\mathrm{s}-\lim _{n \rightarrow \infty} x_{n}$, então $x \in L(f ; \alpha)$.

Para mostrarmos a condição $i$ ) dessa proposição, fixemos $x \in E \operatorname{com} f(x)=\alpha$. Se $\alpha \neq 0$, existe $n_{0} \in \mathbb{N}$ tal que $n>n_{0} \Rightarrow f_{n}(x) \neq 0$, uma vez que $f(x)=\lim _{n \rightarrow \infty} f_{n}(x)$. Para cada $n>n_{0}$, seja $x_{n}=\frac{\alpha}{f_{n}(x)} x$. É claro que $x_{n} \in L\left(f_{n} ; \alpha\right)$, pois:

$$
f_{n}\left(x_{n}\right)=f_{n}\left(\frac{\alpha}{f_{n}(x)} x\right)=\frac{\alpha}{f_{n}(x)} f_{n}(x)=\alpha .
$$
satisfeita.

Além disso, é fácil ver que $x=\mathrm{s}-\lim _{n \rightarrow \infty} x_{n}$, pois $f_{n}(x) \rightarrow f(x)=\alpha$. Logo, a condição $i$ ) está

Se $\alpha=0$, tome $a \in E \operatorname{com} f(a)=1$ e escolha seqüências $\left(z_{n}\right)_{n \in \mathbb{N}}$ e $\left(w_{n}\right)_{n \in \mathbb{N}}$ em $E$ tais que:

(a) $x+a=\mathrm{s}-\lim _{n \rightarrow \infty} z_{n}$

(b) $x-a=\mathrm{s}-\lim _{n \rightarrow \infty} w_{n}$;

(c) $z_{n} \in L\left(f_{n} ; 1\right), \forall n \in \mathbb{N}$;

(d) $w_{n} \in L\left(f_{n} ;-1\right), \forall n \in \mathbb{N}$.

Então, para cada $n \in \mathbb{N}, \eta_{n}=\frac{z_{n}+w_{n}}{2} \in L\left(f_{n} ; 0\right)$, pois:

$$
f_{n}\left(\eta_{n}\right)=\frac{1}{2} f_{n}\left(z_{n}+w_{n}\right)=\frac{1}{2}\left(f_{n}\left(z_{n}\right)+f_{n}\left(w_{n}\right)\right)=0 .
$$

$E$, pelos itens (a) e (b), segue que:

$$
x=\mathrm{s}-\lim _{n \rightarrow \infty} \frac{z_{n}+w_{n}}{2} .
$$

Logo, a condição $i)$ está satisfeita, e $L(f ; \alpha)=\mathrm{K}-\lim _{n \in \mathbb{N}} L\left(f_{n} ; \alpha\right)$.

Um corolário imediato desse teorema está colocado a seguir. Note-se que, assumindo a hipótese de que $E$ é um espaço de Banach, podemos dispor do Princípio da Limitação Uniforme (vide teorema 1.11), o que nos permite enfraquecer a condição (3) do teorema anterior.

Corolário 3.5. Sejam $E$ um espaço de Banach, $\left(f_{n}\right)_{n \in \mathbb{N}}$ uma seqüência de funcionais não-nulos em $E^{\prime}$ e $f \in E^{\prime} \backslash\{0\}$. São equivalentes:

1. $f=\mathrm{w}^{\star}-\lim _{n \rightarrow \infty} f_{n}$;

2. Para cada $\alpha \in \mathbb{R}, L(f ; \alpha)=\mathrm{K}-\lim _{n \in \mathbb{N}} L\left(f_{n} ; \alpha\right)$. 
Demonstração. Inicialmente, suponhamos que $f=\mathrm{w}^{\star}-\lim _{n \rightarrow \infty} f_{n}$. Assim, para cada $x \in E$, a seqüência $\left(\left|f_{n}(x)\right|\right)_{n \in \mathbb{N}}$ é limitada. Pelo teorema 1.11, segue que $\left(\left\|f_{n}\right\|\right)_{n \in \mathbb{N}}$ é limitada. Finalmente, pelo teorema 3.4, segue que para cada $\alpha \in \mathbb{R}, L(f ; \alpha)=\mathrm{K}-\lim _{n \in \mathbb{N}} L\left(f_{n} ; \alpha\right)$.

Reciprocamente, se para cada $\alpha \in \mathbb{R}, L(f ; \alpha)=\mathrm{K}-\lim _{n \in \mathbb{N}} L\left(f_{n} ; \alpha\right)$, o teorema 3.4 garante que $f=\mathrm{w}^{\star}-\lim _{n \rightarrow \infty} f_{n}$.

Além disso, ainda é possível enfraquecer, também, a condição (1) do teorema, de acordo com a seguinte observação:

Observação 3.6. A condição (1) do teorema 3.4 pode ser substituída por uma condição mais fraca, a saber: $L(f ; 1)=\mathrm{K}-\lim _{n \in \mathbb{N}} L\left(f_{n} ; 1\right)$. Na realidade, pode-se fazer isso para qualquer valor não-nulo de $\alpha$. De fato, basta notar que $L(f ; \alpha)=\alpha L(f ; 1), \forall \alpha \neq 0$. É importante notar que essa observação se estende aos resultados a seguir.

No entanto, a hipótese única de que $L(f ; 0)=\mathrm{K}-\lim _{n \in \mathbb{N}} L\left(f_{n} ; 0\right)$ não garante a veracidade do teorema 3.4, como mostra o seguinte exemplo:

Exemplo 3.7. A condição única de que $L(f ; 0)=\mathrm{K}-\lim _{n \in \mathbb{N}} L\left(f_{n} ; 0\right)$ não garante a convergência fraca- $\star$ de $\left(f_{n}\right)_{n \in \mathbb{N}}$ a $f \in E^{\prime}$.

Seja $f \neq 0$ um funcional em $E^{\prime}$. Para cada $n \in \mathbb{N}$, seja $f_{n}=n f$. Vamos mostrar que $L(f ; 0)=\mathrm{K}-\lim _{n \in \mathbb{N}} L\left(f_{n} ; 0\right)$. Seja $x \in L(f ; 0)$, ou seja, $f(x)=0$. Para cada $n \in \mathbb{N}$, seja $x_{n}=x$. Assim, $f_{n}\left(x_{n}\right)=n f(x)=0$, donde $x_{n} \in L\left(f_{n} ; 0\right), \forall n \in \mathbb{N}$.

Agora, seja $N \subseteq \mathbb{N}$ um subconjunto infinito em $\mathbb{N}$. Suponhamos que $x_{n} \in L\left(f_{n} ; 0\right), \forall n \in N$, e que $x_{n} \rightarrow x \in E$. Como $x_{n} \in L\left(f_{n} ; 0\right)$, temos que $f_{n}\left(x_{n}\right)=0, \forall n \in N$. Mas então $x_{n} \rightarrow x$ e $f\left(x_{n}\right)=0, \forall n \in N$, donde $f(x)=0$ e $x \in L(f ; 0)$.

Assim, $L(f ; 0)=\mathrm{K}-\lim _{n \in \mathbb{N}} L\left(f_{n} ; 0\right)$. Mas tome $x \in E$ tal que $f(x)=1$. Então, $f_{n}(x)=n f(x)=$ $n$, e $f_{n}(x) \nrightarrow f(x)$. Logo, $f \neq \mathrm{w}^{\star}-\lim _{n \rightarrow \infty} f_{n}$.

Da mesma forma, a completude de $E$ é essencial para que o corolário apresentado seja válido. O exemplo a seguir ilustra esse fato:

Exemplo 3.8. Se $E$ não é completo, o corolário 3.5 não é verdadeiro, isto é, a convergência fraca$\star$ de uma seqüência de funcionais não garante a convergência, no sentido de Kuratowski, de seus conjuntos de nível.

Seja $\left(e_{n}\right)_{n \in \mathbb{N}}$ a base ortonormal canônica de $l_{2}(\mathbb{R})$, e seja $E \subset l_{2}(\mathbb{R})$ o subespaço das seqüências cujos termos são nulos a partir de um certo índice.

Para cada $n \in \mathbb{N}$, seja $f_{n} \in E^{\prime}$ dado por $f_{n}(x)=x_{1}+n x_{n}$. Como as seqüências de $E$ se anulam 
a partir de um certo índice, temos, claramente, $f=\mathrm{w}^{\star}-\lim _{n \rightarrow \infty} f_{n}$, onde $f(x)=x_{1}$.

Para cada $n \in \mathbb{N}, \frac{e_{n}}{n} \in L\left(f_{n} ; 1\right)$. Mas $0=\mathrm{s}-\lim _{n \rightarrow \infty} \frac{e_{n}}{n}$, donde $\left(L\left(f_{n} ; 1\right)\right)_{n \in \mathbb{N}}$ não pode ser convergente, no sentido de Kuratowski, a $L(f ; 1)$.

Vamos, agora, apresentar uma caracterização da convergência fraca-^ de funcionais lineares contínuos a partir da convergência, no sentido de Wijsman, de seus conjuntos de nível. Para isso, precisaremos do seguinte lema:

Lema 3.9. Seja $E$ um espaço normado. Para cada $x \in E, \alpha \in \mathbb{R}$ e $0 \neq f \in E^{\prime}$, vale:

$$
d(x, L(f ; \alpha))=\frac{|f(x)-\alpha|}{\|f\|} .
$$

Demonstração. Fixemos $\alpha=0$, e tome $x \notin L(f ; 0)$. Pelo corolário 1.10 , existe $g \in E^{\prime}$, com $\|g\|=1$, tal que $\operatorname{ker}(g)=L(f ; 0)$, e $g(x)=d(x, L(f ; 0))$. Assim, $\operatorname{ker}(g)=L(f ; 0)=\operatorname{ker}(f)$. Isso nos permite concluir, pelo corolário 1.5 , que $f=\lambda g$, para algum $\lambda \in \mathbb{R}$. Como $\|g\|=1$, é claro que $g=\frac{f}{\|f\|}$ ou $g=-\frac{f}{\|f\|}$. E, como $g(x) \geq 0$ :

$$
d(x, L(f ; 0))=\frac{|f(x)|}{\|f\|} .
$$

Suponhamos, agora, $\alpha \neq 0$. A observação 3.3 garante que, se $x_{0} \in E$ é tal que $f\left(x_{0}\right)=\alpha$, então $L(f ; \alpha)=x_{0}+L(f ; 0)$. Se $x \in L(f ; \alpha)$, não há o que demonstrar. Caso contrário, temos:

$$
d(x, L(f ; \alpha))=d\left(x, x_{0}+L(f ; 0)\right)=d\left(x-x_{0}, L(f ; 0)\right) .
$$

Portanto,

$$
d(x, L(f ; \alpha))=\frac{\left|f\left(x-x_{0}\right)\right|}{\|f\|}=\frac{\left|f(x)-f\left(x_{0}\right)\right|}{\|f\|}=\frac{|f(x)-\alpha|}{\|f\|}
$$

O próximo teorema caracteriza a convergência fraca- $\star$ de funcionais lineares contínuos a partir da convergência, no sentido de Wijsman, de seus conjuntos de nível. Sua demonstração é simples, em função do lema anterior:

Teorema 3.10. Sejam $E$ um espaço normado, $\left(f_{n}\right)_{n \in \mathbb{N}}$ uma seqüência de funcionais não-nulos em $E^{\prime}$ e $f \in E^{\prime} \backslash\{0\}$. São equivalentes:

1. $f=\mathrm{w}^{\star}-\lim _{n \rightarrow \infty} f_{n}$ e $\|f\|=\lim _{n \rightarrow \infty}\left\|f_{n}\right\|$;

2. Para cada $\alpha \in \mathbb{R}, L(f ; \alpha)=\mathrm{W}-\lim _{n \in \mathbb{N}} L\left(f_{n} ; \alpha\right)$. 
Demonstração. (1) $\Rightarrow(2)$ Devemos mostrar que, para cada $\alpha \in \mathbb{R}$, a seqüência $\left(d\left(\cdot, L\left(f_{n} ; \alpha\right)\right)\right)_{n \in \mathbb{N}}$ converge pontualmente a $d(\cdot, L(f ; \alpha))$. Tome $x \in E$. Pelo lema 3.9 e pela condição (1), temos:

$$
\lim _{n \rightarrow \infty} d\left(x, L\left(f_{n} ; \alpha\right)\right)=\lim _{n \rightarrow \infty} \frac{\left|f_{n}(x)-\alpha\right|}{\left\|f_{n}\right\|}=\frac{|f(x)-\alpha|}{\|f\|}=d(x, L(f ; \alpha)) .
$$

Logo, $L(f ; \alpha)=\mathrm{W}-\lim _{n \in \mathbb{N}} L\left(f_{n} ; \alpha\right)$.

(2) $\Rightarrow$ (1) Sabe-se que a convergência no sentido de Wijsman garante a convergência no sentido de Kuratowski de uma seqüência de conjuntos. Dessa forma, se assumirmos (2), temos, pelo teorema 3.4, $f=\mathrm{w}^{\star}-\lim _{n \rightarrow \infty} f_{n}$.

Além disso, se $g \in E^{\prime}$ e $g \neq 0$, temos, pelo lema 3.9:

$$
d(0, L(g ; 1))=\frac{|g(0)-1|}{\|g\|}=\frac{1}{\|g\|} .
$$

Por $(2), d(0, L(f ; 1))=\lim _{n \rightarrow \infty} d\left(0, L\left(f_{n} ; 1\right)\right)$. Dessa forma, fazendo $g_{n}=f$ e também $g=f$ :

$$
\frac{1}{\|f\|}=\lim _{n \rightarrow \infty} \frac{1}{\left\|f_{n}\right\|}
$$

donde $\|f\|=\lim _{n \rightarrow \infty}\left\|f_{n}\right\|$.

\subsection{Convergência em Norma}

A seguir, apresentamos um teorema que relaciona a convergência em norma de funcionais lineares contínuos com a r-convergência de seus conjuntos de nível.

Teorema 3.11. Sejam $E$ um espaço normado, $\left(f_{n}\right)_{n \in \mathbb{N}}$ uma seqüência de funcionais não-nulos em $E^{\prime}$ e $f \in E^{\prime} \backslash\{0\}$. São equivalentes:

1. $f=\mathrm{s}-\lim _{n \rightarrow \infty} f_{n}$;

2. Para cada $\alpha \in \mathbb{R}, L(f ; \alpha)=\mathrm{r}-\lim _{n \in \mathbb{N}} L\left(f_{n} ; \alpha\right)$.

Demonstração. (1) $\Rightarrow(2)$ Sejam $r, \alpha \in \mathbb{R}$ e $\epsilon>0$ fixos. Seja $0<\delta<\inf _{n \rightarrow \infty}\left\|f_{n}\right\|$. Note-se que esse ínfimo é positivo, uma vez que todos os funcionais em questão são não-nulos e convergem a um funcional não-nulo. Por hipótese, $f=s-\lim _{n \rightarrow \infty} f_{n}$, donde:

$$
\exists n_{0} \in \mathbb{N}: n>n_{0}, x \in r B[0,1] \Rightarrow\left|f_{n}(x)-f(x)\right|<\epsilon \delta .
$$

Pela escolha de $\delta$ - observe que $\left\|f_{n}\right\|>\delta$, para cada $n>n_{0}$, temos:

$$
\sup _{x \in B[0, \epsilon]}\left|f_{n}(x)\right|>\epsilon \delta .
$$


Então, $\forall x \in r B[0,1], \forall n>n_{0}$, existe $z=z(x, n) \in \epsilon B[0,1]$ com $f_{n}(z)=f(x)-f_{n}(x)$. De fato, a partir das duas inequações anteriores conclui-se que:

$$
\sup _{x \in B[0, \gamma]}\left|f_{n}(x)\right|>\left|f_{n}(x)-f(x)\right|
$$

onde $\gamma=\min \{r, \epsilon\}$ e $n>n_{0}$. Além disso, $\min _{x \in B[0, \gamma]}\left|f_{n}(x)\right|=0$. Temos, então:

$$
0 \leq\left|f_{n}(x)-f(x)\right|<\sup _{x \in B[0, \gamma]}\left|f_{n}(x)\right|
$$

e, como $f_{n}$ é contínua em $B[0, \gamma]$, segue o resultado. Podemos supor $f_{n}(z)=f(x)-f_{n}(x)$; caso contrário, basta tomar o ponto $-z$.

Se $x \in L(f ; \alpha) \cap r B[0,1]$, então $x+z(x, n) \in L\left(f_{n} ; \alpha\right)$, pois:

$$
f_{n}(x+z(x, n))=f_{n}(x)+f_{n}(z(x, n))=f_{n}(x)+f(x)-f_{n}(x)=f(x)=\alpha .
$$

Mas $\|z(x, n)\| \leq \epsilon$, donde obtemos, se $n>n_{0}$ :

$$
L(f ; \alpha) \cap r B[0,1] \subseteq L\left(f_{n} ; \alpha\right)+\epsilon B[0,1]
$$

Analogamente, mostra-se que, para $n>n_{0}$ :

$$
L\left(f_{n} ; \alpha\right) \cap r B[0,1] \subseteq L(f ; \alpha)+\epsilon B[0,1]
$$

Segue, portanto, que $L(f ; \alpha)=\mathrm{r}-\lim _{n \in \mathbb{N}} L\left(f_{n} ; \alpha\right)$.

$(2) \Rightarrow(1)$ Sabemos que a topologia da convergência uniforme em subconjuntos limitados de $E$ em $C_{b}(E, \mathbb{R})$, quando restrita a $E^{\prime}$, equivale à topologia da norma em $E^{\prime}$. Portanto, é suficiente mostrarmos que a seqüência $\left(f_{n}\right)_{n \in \mathbb{N}}$ converge uniformemente a $f$ em $r B[0,1]$, para cada $r>0$. Fixemos $r>0$.

Para cada $n \in \mathbb{N}$, seja $g_{n}(x)=f_{n}(x)-1$, e defina $g(x)=f(x)-1$. Vamos mostrar que $\left(g_{n}\right)_{n \in \mathbb{N}}$ converge uniformemente a $g$ em $r B[0,1]$. Sabemos que a r-convergência de uma seqüiência de conjuntos implica sua convergência no sentido de Wijsman, e que isso não supõe a reflexividade do espaço. Então, $L(f ; \alpha)=\mathrm{W}-\lim _{n \in \mathbb{N}} L\left(f_{n} ; \alpha\right)$. Assim, de acordo com o teorema 3.10, podemos afirmar que $f=\mathrm{w}^{\star}-\lim _{n \rightarrow \infty} f_{n}$ e $\|f\|=\lim _{n \rightarrow \infty}\left\|f_{n}\right\|$

Como:

$$
d\left(0, L\left(f_{n} ; 1\right)\right)=\frac{1}{\left\|f_{n}\right\|},
$$

temos que $\lim _{n \rightarrow \infty} d\left(0, L\left(f_{n} ; 1\right)\right)$ existe. Assim, a seqüência $\left(d\left(\cdot, L\left(f_{n} ; 1\right)\right)_{n \in \mathbb{N}}\right.$ é uniformemente limitada, quando restrita a $r B[0,1]$. Além disso, pela convergência uniforme de $\left(d\left(\cdot, L\left(f_{n} ; 1\right)\right)_{n \in \mathbb{N}}\right.$ a 
$d(\cdot, L(f ; 1))$, e pela convergência de $\left(\left\|f_{n}\right\|\right)_{n \in \mathbb{N}}$ a $\|f\|$, a seqüência $\left(\left\|f_{n}\right\| d\left(\cdot, L\left(f_{n} ; 1\right)\right)\right)_{n \in \mathbb{N}}$ converge uniformemente a $\|f\| d(\cdot, L(f ; 1))$ em $r B[0,1]$.

Mas:

$$
\left|g_{n}(x)\right|=\left|f_{n}(x)-1\right|=\left|\left\|f_{n}\right\| \frac{\left|f_{n}(x)-1\right|}{\left\|f_{n}\right\|}\right|=\left\|f_{n}\right\| d\left(x, L\left(f_{n} ; 1\right)\right)
$$

$\mathrm{e}$

$$
|g(x)|=|f(x)-1|=\left|\|f\| \frac{|f(x)-1|}{\|f\|}\right|=\|f\| d(x, L(f ; 1))
$$

Logo, $|g(x)|=\lim _{n \rightarrow \infty}\left|g_{n}(x)\right|$ uniformemente em $r B[0,1]$.

Suponhamos, agora, por absurdo, que $\left(g_{n}\right)_{n \in \mathbb{N}}$ não converge uniformemente a $g$ em $r B[0,1]$. Nesse caso, existem $\epsilon>0$ e $N \subseteq \mathbb{N}$ infinito tais que, para cada $n \in N$ :

$$
\sup _{x \in r B[0,1]}\left|g_{n}(x)-g(x)\right|>4 \epsilon \quad \text { e } \quad \sup _{x \in r B[0,1]} \| g_{n}(x)|-| g(x)||<\epsilon .
$$

Pela combinação dessas duas inequações, existe um subconjunto $M \subseteq N$ infinito que satisfaz uma das seguintes asserções:

(a) Para cada $n \in M$, existe $x_{n} \in r B[0,1]$ tal que $g_{n}\left(x_{n}\right)<-\epsilon<\epsilon<g\left(x_{n}\right)$;

(b) Para cada $n \in M$, existe $x_{n} \in r B[0,1]$ tal que $g\left(x_{n}\right)<-\epsilon<\epsilon<g_{n}\left(x_{n}\right)$.

Consideremos o caso (a). Fixe $x_{0} \in r B[0,1] \operatorname{com} g\left(x_{0}\right)>\epsilon$. Como $f=\mathrm{w}^{\star}-\lim _{n \rightarrow \infty} f_{n}$, a seqüência $\left(g_{n}\right)_{n \in \mathbb{N}}$ converge pontualmente a $g$. Assim, existe $n \in M$ tal que $g_{n}\left(x_{0}\right)>\epsilon$. Como $g_{n}\left(x_{n}\right)<-\epsilon$, e $g_{n}$ é um funcional afim, existe $\lambda \in[0,1]$ tal que:

$$
g_{n}\left(\lambda x_{n}+(1-\lambda) x_{0}\right)=0 .
$$

E, como $g$ é linear:

$$
g\left(\lambda x_{n}+(1-\lambda) x_{0}\right)=\lambda g\left(x_{n}\right)+(1-\lambda) g\left(x_{0}\right)>\lambda \epsilon+\epsilon-\lambda \epsilon=\epsilon .
$$

Obtemos, assim, uma contradição, uma vez que, se $\beta=\lambda x_{n}+(1-\lambda) x_{0}$, então:

$$
\beta \in r B[0,1] \quad \text { e } \quad\left\|g_{n}(\beta)|-| g(\beta)\right\|>\epsilon .
$$

Analogamente, o caso (b) nos leva a uma contradição. Concluímos, portanto, que $\left(g_{n}\right)_{n \in \mathbb{N}}$ converge uniformemente a $g$. Logo, $f=\mathrm{s}-\lim _{n \rightarrow \infty} f_{n}$.

Esse teorema nos permite obter mais exemplos de seqüências r-convergentes de conjuntos. 
Exemplo 3.12. Apresentaremos mais dois exemplos de seqüencias de conjuntos r-convergentes:

a) Considere os seguintes planos em $\mathbb{R}^{3}$ :

$$
\begin{gathered}
\pi_{n}:\left(2-\frac{1}{n}\right) x_{1}+\left(1+\frac{1}{n}\right)^{2 n} x_{2}+\left(\frac{1}{3^{n}}\right) x_{3}=1, \forall n \in \mathbb{N} \\
\pi: 2 x_{1}+e^{2} x_{2}=1
\end{gathered}
$$

Afirmamos que $\pi=\mathrm{r}-\lim _{n \in \mathbb{N}} \pi_{n}$. De fato, basta definirmos os seguintes funcionais no dual de $\mathbb{R}^{3}$ :

$$
\begin{gathered}
f_{n}\left(x_{1}, x_{2}, x_{3}\right)=\left(2-\frac{1}{n}\right) x_{1}+\left(1+\frac{1}{n}\right)^{2 n} x_{2}+\left(\frac{1}{3^{n}}\right) x_{3}, \\
f\left(x_{1}, x_{2}, x_{3}\right)=2 x_{1}+e^{2} x_{2} .
\end{gathered}
$$

É fácil ver que $f=\mathrm{s}-\lim _{n \rightarrow \infty} f_{n} . \quad \operatorname{Logo}, L(f ; 1)=\mathrm{r}-\lim _{n \in \mathbb{N}} L\left(f_{n} ; 1\right) . \quad$ Mas $\pi_{n}=L\left(f_{n} ; 1\right) \mathrm{e}$ $\pi=L(f ; 1)$, donde segue o resultado que procurávamos.

b) Considere $\xi_{n}=\left(\xi_{n}^{(1)}, \xi_{n}^{(2)}, \cdots\right), \xi=\left(\xi^{(1)}, \xi^{(2)}, \cdots\right) \in l_{2}(\mathbb{R})$, com $\xi=\mathrm{s}-\lim _{n \rightarrow \infty} \xi_{n}$. Definamos:

$$
f_{n}(x)=\sum_{i=0}^{\infty} \xi_{n}^{(i)} x^{(i)} \quad \text { e } \quad f(x)=\sum_{i=0}^{\infty} \xi^{(i)} x^{(i)}
$$

Temos que $f=\mathrm{s}-\lim _{n \rightarrow \infty} f_{n}$. Logo, $L(f ; 1)=\mathrm{r}-\lim _{n \in \mathbb{N}} L\left(f_{n} ; 1\right)$.

Em particular, se $\xi_{n}=\left(1, \frac{1}{2}, \frac{1}{3}, \cdots, \frac{1}{n}, 0,0, \cdots\right)$ e $\xi=\left(1, \frac{1}{2}, \frac{1}{3}, \cdots\right)$, temos que:

$$
\begin{gathered}
A_{n}=L\left(f_{n} ; 1\right)=\left\{x \in l_{2}(\mathbb{R}): x^{(1)}+\frac{x^{(2)}}{2}+\cdots+\frac{x^{(n)}}{n}=1\right\}, \\
A=L(f ; 1)=\left\{x \in l_{2}(\mathbb{R}): x^{(1)}+\frac{x^{(2)}}{2}+\frac{x^{(3)}}{3}+\cdots=1\right\}
\end{gathered}
$$

Então, $A=\mathrm{r}-\lim _{n \in \mathbb{N}} A_{n}$.

Em função da grande similaridade das definições de convergência de conjuntos no sentido de Kuratowski e no sentido de Mosco, o artigo [3] busca estabelecer, para seqüências de conjuntos convergentes no sentido de Mosco, um resultado análogo àquele obtido no caso de uma seqüência convergente no sentido de Kuratowski, apresentado no teorema 3.4.

A seguir, caminharemos na direção do resultado procurado. É importante notar que sua demonstração usa algumas variações do que é feito na demonstração do teorema 3.4. No entanto, nesse caso, a reflexividade do espaço $E$ é fundamental. Iniciamos com o seguinte: 
Lema 3.13. Sejam $E$ um espaço normado, $\left(f_{n}\right)_{n \in \mathbb{N}}$ uma seqüência de funcionais não-nulos em $E^{\prime}$ e $f \in E^{\prime} \backslash\{0\}$. São equivalentes:

1. Para cada $\alpha \in \mathbb{R}, L(f ; \alpha)=\mathrm{M}-\lim _{n \in \mathbb{N}} L\left(f_{n} ; \alpha\right)$;

2. Para cada $N \subseteq \mathbb{N}$ infinito, as condiçôes $x=\mathrm{w}-\lim _{n \in N} x_{n}$ e $\alpha=\lim _{n \in N} f_{n}\left(x_{n}\right)$ implicam $f(x)=\alpha$. Demonstração. (1) $\Rightarrow(2)$ Suponha $x=\mathrm{w}-\lim _{n \rightarrow \infty} x_{n}$ e $\alpha=\lim _{n \in N} f_{n}\left(x_{n}\right)$. Escolha $z \in X$ tal que $f(z)=1-\alpha$. Por (1), escolha $\left(z_{n}\right)_{n \in \mathbb{N}} \operatorname{com} z=s-\lim _{n \rightarrow \infty} z_{n}$ tal que, para cada $n \in \mathbb{N}$, $z_{n} \in L\left(f_{n} ; 1-\alpha\right)$. Observe que:

$$
\lim _{n \in N} f\left(x_{n}+z_{n}\right)=\lim _{n \in N} f\left(x_{n}\right)+\lim _{n \in N} f\left(z_{n}\right)=\alpha+1-\alpha=1 .
$$

Se escrevermos $\alpha_{n}=f_{n}\left(x_{n}+z_{n}\right)$, então existe $n_{0} \in \mathbb{N}$ tal que, se $n>n_{0}$ :

$$
\frac{x_{n}+z_{n}}{\alpha_{n}} \in L\left(f_{n} ; 1\right) \quad \text { e } \quad x+z=\mathrm{w}-\lim _{n \rightarrow \infty}\left(x_{n}+z_{n}\right)
$$

Por (1), $x+z \in L(f ; 1)$, donde $f(x+z)=1$, e $f(x)=1-f(z)=1-1+\alpha=\alpha$.

$(2) \Rightarrow(1)$ Pela segunda condição da proposição 2.31, devemos ter: sempre que $N \subseteq \mathbb{N}$ é infinito e $x_{n} \in L\left(f_{n} ; \alpha\right), \forall n \in N$, a convergência fraca de $\left(x_{n}\right)_{n \in N}$ a $x \in X$ implica que $x \in L(f ; \alpha)$. Por (2), $f(x)=\alpha$, donde $x \in L(f ; \alpha)$, e essa condição está satisfeita.

Além disso, note que a condição (2) implica a condição (2) do teorema 3.4, uma vez que a convergência em norma implica a convergência fraca de uma seqüência. E esse teorema garante que $L(f ; \alpha)=\mathrm{K}-\lim _{n \in \mathbb{N}} L\left(f_{n} ; \alpha\right)$

Mas a primeira condição da proposição 2.16 implica a primeira condição da proposição 2.31 . Logo, $L(f ; \alpha)=\mathrm{M}-\lim _{n \in \mathbb{N}} L\left(f_{n} ; \alpha\right)$.

O teorema seguinte usa o lema anterior e faz analogia ao teorema 3.4. Observe que a reflexividade do espaço é utilizada na implicação $(2) \Rightarrow(3)$.

Teorema 3.14. Sejam $E$ um espaço de Banach reflexivo, $\left(f_{n}\right)_{n \in \mathbb{N}}$ uma seqüência de funcionais não-nulos em $E^{\prime}$ e $f \in E^{\prime} \backslash\{0\}$. São equivalentes:

1. Para cada $\alpha \in \mathbb{R}, L(f ; \alpha)=\mathrm{M}-\lim _{n \in \mathbb{N}} L\left(f_{n} ; \alpha\right)$;

2. Para cada $N \subseteq \mathbb{N}$ infinito, as condições $x=\mathrm{w}-\lim _{n \in N} x_{n}$ e $\alpha=\lim _{n \in N} f_{n}\left(x_{n}\right)$ implicam $f(x)=\alpha$;

3. $f=\mathrm{s}-\lim _{n \rightarrow \infty} f_{n}$

4. Sempre que $x=\mathrm{w}-\lim _{n \rightarrow \infty} x_{n}, f(x)=\lim _{n \rightarrow \infty} f_{n}\left(x_{n}\right)$. 
Demonstração. $(1) \Rightarrow$ (2) Segue do lema 3.13 .

$(2) \Rightarrow(3)$ Vamos assumir (2). Pelo teorema 3.4, a sequiência $\left(\left\|f_{n}\right\|\right)_{n \in \mathbb{N}}$ é limitada. Se assumir$\operatorname{mos} f \neq \mathrm{s}-\lim _{n \rightarrow \infty} f_{n}$, existem $\epsilon>0$ e $N \subseteq \mathbb{N}$ infinito tais que, para cada $n \in N,\left\|f_{n}-f\right\|>\epsilon$. Escolha $x_{n} \in E$ com $\left\|x_{n}\right\|=1$ e $\left|f_{n}\left(x_{n}\right)-f\left(x_{n}\right)\right|>\epsilon$. Observe que esse vetor existe, uma vez que:

$$
\left\|f_{n}-f\right\|=\sup _{\|x\|=1}\left|f_{n}(x)-f(x)\right|>\epsilon .
$$

Como $E$ é reflexivo, sua bola unitária é fracamente compacta (vide [12], página 245). Pelo Teorema de Eberlein (vide [12], página 248), a bola é fracamente seqüencialmente compacta. Dessa forma, toda seqüência na bola deve possuir uma subseqüência fracamente convergente. Além disso, como a seqüência $\left(\left|f_{n}\left(x_{n}\right)\right|\right)_{n \in N}$ é limitada, pois $\left(\left\|f_{n}\right\|\right)_{n \in \mathbb{N}}$ o é, $\left(f_{n}\left(x_{n}\right)\right)_{n \in N}$ também deve possuir subsequiência convergente. Podemos, então, afirmar que existem $M \subseteq N$ subconjunto infinito, $x \in B[0,1]$ e $\alpha \in \mathbb{R}$ tais que $x=\mathrm{w}-\lim _{n \in M} x_{n}$ e $\alpha=\lim _{n \in M} f_{n}\left(x_{n}\right)$.

Como $x=\mathrm{w}-\lim _{n \in M} x_{n}$, segue que $f(x)=\lim _{n \in M} f_{n}\left(x_{n}\right)$. Além disso, temos $\left|f_{n}\left(x_{n}\right)-f\left(x_{n}\right)\right|>\epsilon$, $\forall n \in M$, donde $|\alpha-f(x)| \geq \epsilon$, contradição.

(3) $\Rightarrow$ (4) Suponhamos que $f=s-\lim _{n \rightarrow \infty} f_{n}$. Observe que:

$$
\left|f_{n}\left(x_{n}\right)-f(x)\right| \leq\left|f_{n}\left(x_{n}\right)-f\left(x_{n}\right)\right|+\left|f\left(x_{n}\right)-f(x)\right| \leq\left\|f_{n}-f\right\|\left\|x_{n}\right\|+\left|f\left(x_{n}\right)-f(x)\right| .
$$

Por hipótese, $\left\|f_{n}-f\right\| \rightarrow 0$. Se assumirmos que $x=\mathrm{w}-\lim _{n \rightarrow \infty} x_{n}$, temos $f\left(x_{n}\right) \rightarrow f(x)$. Logo, $\left\|f_{n}\left(x_{n}\right)-f(x)\right\| \rightarrow 0$, e $f(x)=\lim _{n \rightarrow \infty} f_{n}\left(x_{n}\right)$.

(4) $\Rightarrow$ (2) Assumindo (4), se $x=\mathrm{w}-\lim _{n \rightarrow \infty} x_{n}$, então $f(x)=\lim _{n \rightarrow \infty} f_{n}\left(x_{n}\right)$. Se $\alpha=\lim _{n \in N} f_{n}\left(x_{n}\right)$, então $f(x)=\alpha$.

$(2) \Rightarrow(1)$ Segue do lema 3.13 .

De acordo com o teorema 2.35, a convergência no sentido de Mosco de uma seqüência de conjuntos convexos implica a convergência, no sentido de Wijsman, dessa seqüência. No entanto, antes da elaboração do artigo [3], não se sabia se a convergência no sentido de Mosco de uma seqüência de conjuntos fechados e convexos em um espaço reflexivo poderia ser estritamente mais forte que a convergência, no sentido de Wijsman, dessa mesma seqüência. No artigo [3], mostra-se que isso é possível. A seguir, vamos apresentar a maneira como isso é feito.

No artigo [4], A. L. Brown constrói um espaço reflexivo e estritamente convexo $F$ que não possui a propriedade de Kadec, isto é, no qual a combinação da convergência fraca e da convergência das normas de um seqüência não garante sua convergência em norma. 
Esse espaço nada mais é do que o espaço $l_{2}(\mathbb{R})$ das seqüências reais com quadrado somável, munido de uma norma convenientemente criada para satisfazer certas propriedades. Dessa forma, é possível obter uma seqüência $\left(y_{n}\right)_{n \in \mathbb{N}}$ em $F$ com:

- $y=\mathrm{w}-\lim _{n \rightarrow \infty} y_{n}$ e $\|y\|=\lim _{n \rightarrow \infty}\left\|y_{n}\right\| ;$

- $y \neq s-\lim _{n \rightarrow \infty} y_{n}$.

O teorema seguinte apresenta o resultado obtido:

Teorema 3.15. Existem um espaço reflexivo $E$, uma seqüência de conjuntos fechados e convexos $\left(A_{n}\right)_{n \in \mathbb{N}} \subset E$ e um conjunto fechado e convexo $A \in E$ tais que $A=\mathrm{W}-\lim _{n \in \mathbb{N}} A_{n}$, mas $A \neq$ $\mathrm{M}-\lim _{n \in \mathbb{N}} A_{n}$.

Demonstração. Considere $E=F^{\prime}$, onde $F$ é o espaço construído em [4]. Observe que $E$ é obviamente reflexivo e que $E^{\prime}=F^{\prime \prime}=F$. Dessa forma, podemos enxergar $y, y_{1}, y_{2}, \cdots$ como funcionais lineares contínuos no espaço reflexivo $E$.

Como $E$ é reflexivo, a convergência fraca e a convergência fraca- $\star$ de funcionais são equivalentes. Logo:

1. $y=\mathrm{w}^{\star}-\lim _{n \rightarrow \infty} y_{n}$ e $\|y\|=\lim _{n \rightarrow \infty}\left\|y_{n}\right\|$

2. $y \neq \mathrm{s}-\lim _{n \rightarrow \infty} y_{n}$.

Então:

- Por (1) e pelo teorema $3.10, L(f ; 1)=\mathrm{W}-\lim _{n \in \mathbb{N}} L\left(f_{n} ; 1\right)$;

- Por (2) e pelo teorema $3.14, L(f ; 1) \neq \mathrm{M}-\lim _{n \in \mathbb{N}} L\left(y_{n} ; 1\right)$.

Dessa forma, $E$ é um espaço reflexivo e estritamente convexo no qual a convergência, no sentido de Wijsman, de conjuntos convexos e fechados não acarreta a convergência dessa seqüência no sentido de Mosco.

Sabemos também que a r-convergência de uma seqüência de conjuntos fechados e convexos pode ser estritamente mais forte que a convergência no sentido de Mosco dessa seqüência (exemplo 2.36). No entanto, se trabalharmos apenas com hiperplanos, isso não pode ocorrer. Vamos demonstrar esse fato.

Lema 3.16. Seja $E$ um espaço normado, e seja $H \subseteq E$ um hiperplano fechado. Para cada $x \in E \backslash H$, existe $f \in E^{\prime}, f \neq 0$, tal que $x+H=L(f ; 1)$. 
Demonstração. Sabemos que, para cada hiperplano $H$ de $E$, existe $\phi \in E^{\prime}$ tal que $H=\operatorname{ker} \phi$. Seja $x \in E \backslash H$. Então, $\phi(x)=\alpha \neq 0$.

Considere $f=\alpha^{-1} \phi$. É óbvio que $x+H \subseteq L(f ; 1)$. Por outro lado, se $y \in L(f ; 1)$, então $f(y)=1$, donde $\phi(y)=\alpha$ e, portanto, $\phi(x-y)=0$. Logo, $x-y \in H$, e $y \in x+H$. Segue que $L(f ; 1) \subseteq x+H$.

Proposição 3.17. Sejam $E$ um espaço de Banach reflexivo, $\left(H_{n}\right)_{n \in \mathbb{N}}$ uma seqüência de hiperplanos fechados em $E$ e $H$ um hiperplano fechado em $E$. Então:

$$
H=\mathrm{M}-\lim _{n \in \mathbb{N}} H_{n} \Leftrightarrow H=\mathrm{r}-\lim _{n \in \mathbb{N}} H_{n} .
$$

Demonstração. Pelo teorema 2.35, sabemos que $H=\mathrm{r}-\lim _{n \in \mathbb{N}} H_{n} \Rightarrow H=\mathrm{M}-\lim _{n \in \mathbb{N}} H_{n}$. Resta-nos, então, provar a outra implicação.

Suponhamos que $H=\mathrm{M}-\lim _{n \in \mathbb{N}} H_{n}$, e seja $F=\left(\bigcup_{n \in \mathbb{N}} H_{n}\right) \cup H$. Pelo Teorema de Baire (vide [12], página 36), $F$ tem interior vazio. Logo, existe $x \in E \backslash F$, donde $x \notin H_{n}, \forall n \in \mathbb{N}$ e $x \notin H$.

Pelo lema 3.16, para cada $n \in \mathbb{N}$, existe $f_{n} \in E^{\prime}, f_{n} \neq 0$, tal que $x+H_{n}=L\left(f_{n} ; 1\right)$. Além disso, existe $f \in E^{\prime}, f \neq 0$, tal que $x+H=L(f ; 1)$.

Pelo lema 2.37, $x+H=\mathrm{M}-\lim _{n \in \mathbb{N}}\left(x+H_{n}\right)$. Então, $L(f ; 1)=\mathrm{M}-\lim _{n \in \mathbb{N}} L\left(f_{n} ; 1\right)$. Assim, pelo teorema 3.14, temos $f=\mathrm{s}-\lim _{n \rightarrow \infty} f_{n}$. Pelo teorema 3.11 , segue que $L(f ; 1) \stackrel{n \in \mathbb{N}}{=} \mathrm{r}-\lim _{n \in \mathbb{N}} L\left(f_{n} ; 1\right)$, donde $x+H=\mathrm{r}-\lim _{n \in \mathbb{N}}\left(x+H_{n}\right)$. Mas então $H=\mathrm{r}-\lim _{n \in \mathbb{N}} H_{n}$, e o resultado está provado.

Os teoremas 3.4 e 3.14 contrastam as convergências, nos sentidos de Mosco e de Kuratowski, de sequiências de hiperplanos em um espaço reflexivo. Terminamos o capítulo com um exemplo concreto:

Exemplo 3.18. Vamos mostrar que a convergência fraca- $\star$ de uma seqüência de funcionais no dual de $l_{2}(\mathbb{R})$, embora assegure a convergência, no sentido de Kuratowski, de seus conjuntos de nível, não garante essa convergência, no sentido de Mosco. Em virtude do teorema 3.4, está implícita, na construção a seguir, uma seqüência de hiperplanos em $l_{2}(\mathbb{R})$ que converge no sentido de Kuratowski, mas não no de Mosco.

Considere, em $l_{2}^{\prime}(\mathbb{R})$, a seqüência $f_{n}=e_{1}^{\star}+e_{n}^{\star}, \forall n \in \mathbb{N}$, onde $e_{i}^{\star}$ é a i-ésima projeção de um elemento de $l_{2}(\mathbb{R})$. Observe que:

1. A seqüência $\left(f_{n}\right)_{n \in \mathbb{N}}$ é limitada em norma;

2. Se $f=e_{1}^{\star}$, então $f=\mathrm{w}^{\star}-\lim _{n \rightarrow \infty} f_{n}$. 
Pelo teorema 3.4 , temos que $L(f ; 1)=\mathrm{K}-\lim _{n \in \mathbb{N}} L\left(f_{n} ; 1\right)$. Para cada $n \geq 2, e_{n} \in L\left(f_{n} ; 1\right)$. Mas $0=\mathrm{w}-\lim _{n \rightarrow \infty} e_{n}$. Assim, $L(f ; 1) \neq \mathrm{M}-\lim _{n \in \mathbb{N}} L\left(f_{n} ; 1\right)$, uma vez que, se $L(f ; 1)=\mathrm{M}-\lim _{n \in \mathbb{N}} L\left(f_{n} ; 1\right)$, teríamos $f(0)=\lim _{n \rightarrow \infty} f_{n}\left(e_{n}\right)=1$, absurdo. 


\section{Capítulo 4}

\section{Polinômios Homogêneos}

Ao longo desse capítulo, apresentaremos as definições e alguns resultados básicos da teoria de polinômios homogêneos em espaços de Banach. Com isso, fundamentaremos as ferramentas utilizadas no capítulo 5, que relaciona os diversos tipos de convergência de conjuntos com a convergência desses polinômios. A maior parte desse capítulo se baseia na dissertação [15].

\subsection{Aplicações Multilineares}

Nessa seção, estudaremos as aplicações multilineares em espaços normados, e provaremos alguns resultados sobre sua continuidade. O estudo dessas aplicações servirá como base para o estudo dos polinômios homogêneos.

Em função de estarmos interessados apenas em aplicações contínuas, enunciaremos os resultados no contexto de espaços normados. No entanto, alguns resultados continuam válidos mesmo no contexto de espaços vetoriais.

Definição 4.1. Sejam $n \in \mathbb{N}, E_{1}, E_{2}, \cdots, E_{n}$ e $F$ espaços normados sobre o corpo $\mathbb{K}$. Uma aplicação $A: E_{1} \times E_{2} \times \cdots \times E_{n} \longrightarrow F$ é dita $n$-linear se é linear em cada uma de suas variáveis.

$O$ conjunto de todas as aplicações $n$-lineares em um espaço se torna um espaço vetorial quando munido das operações usuais envolvendo funções. Denotaremos por $L_{a}\left(E_{1}, \cdots, E_{n} ; F\right)$ o espaço de todas as aplicações $n$-lineares de $E_{1}, E_{2}, \cdots, E_{n}$ em $F$. Quando $F=\mathbb{K}$, escrevemos simplesmente $L_{a}\left(E_{1}, \cdots, E_{n}\right)$. No caso em que $E_{1}=E_{2}=\cdots=E_{n}=E$, escrevemos $L_{a}\left({ }^{n} E ; F\right)$. Por convenção, $L_{a}\left({ }^{0} E ; F\right)=F$.

Se $E_{1}, \cdots, E_{n}$ são espaços normados sobre o corpo $\mathbb{K}$, o espaço produto $E_{1} \times \cdots \times E_{n}$ também 
é um espaço normado, com qualquer uma das normas:

$$
\begin{gathered}
\|x\|_{\infty}=\max _{1 \leq i \leq n}\left\|x_{i}\right\| \\
\|x\|_{p}=\left(\sum_{i=1}^{n}\left\|x_{i}\right\|^{p}\right)^{\frac{1}{p}}, \quad 1 \leq p<+\infty
\end{gathered}
$$

Ambas as normas são equivalentes e geram a topologia produto em $E_{1} \times \cdots \times E_{n}$. Ao longo do texto, utilizaremos a norma $\|\cdot\|_{\infty}$, que será denotada simplesmente por $\|\cdot\|$.

A proposição seguinte apresenta algumas equivalências para a continuidade de uma aplicação multilinear. Essa proposição é uma generalização de resultado análogo para as aplicações lineares:

Proposição 4.2. Sejam $n \in \mathbb{N}, E_{1}, E_{2}, \cdots, E_{n}$ e $F$ espaços normados sobre o corpo $\mathbb{K}$ e $A$ : $E_{1} \times E_{2} \times \cdots \times E_{n} \rightarrow F$ uma aplicação $n$-linear. São equivalentes:

1. A é contínua;

2. A é contínua em 0 ;

3. Existe $M>0$ tal que $\left\|A\left(x_{1}, \cdots, x_{n}\right)\right\| \leq M\left\|x_{1}\right\| \cdots\left\|x_{n}\right\|, \forall\left(x_{1}, \cdots, x_{n}\right) \in E_{1} \times \cdots \times E_{n}$.

Demonstração. $(1) \Rightarrow(2)$ : Óbvio.

$(2) \Rightarrow(3)$ : Se $A$ é contínua no ponto 0 , dado $\epsilon=1$ existe $\delta>0$ tal que, se $\|x\|<\delta$, então $\|A(x)\| \leq 1$. Seja $x \in E_{1} \times E_{2} \times \cdots \times E_{n}$ tal que $x_{i} \neq 0, \forall 1 \leq i \leq n$, e definamos:

$$
y=\left(\frac{\delta x_{1}}{2\left\|x_{1}\right\|}, \frac{\delta x_{2}}{2\left\|x_{2}\right\|}, \cdots, \frac{\delta x_{n}}{2\left\|x_{n}\right\|}\right) .
$$

Então, $\|y\|<\delta$ e $\|A(y)\| \leq 1$, donde:

$$
\left\|A\left(x_{1}, \cdots, x_{n}\right)\right\| \leq \frac{2^{n}}{\delta^{n}}\left\|x_{1}\right\| \cdots\left\|x_{n}\right\| .
$$

Se $x \in E_{1} \times E_{2} \times \cdots \times E_{n}$ é tal que $x_{i}=0$, para algum $i$, então $A(x)=0$, e a desigualdade continua válida.

$(3) \Rightarrow(1)$ : Mostremos que $A$ é uniformemente contínua sobre os limitados e, portanto, é contínua. Fixe $a=\left(a_{1}, \cdots, a_{n}\right) \in E_{1} \times E_{2} \times \cdots \times E_{n}$, e seja $x=\left(x_{1}, \cdots, x_{n}\right) \in E_{1} \times E_{2} \times \cdots \times E_{n}$. Então:

$$
A(x)-A(a)=A\left(x_{1}-a_{1}, x_{2}, \cdots, x_{n}\right)+\cdots+A\left(a_{1}, \cdots, a_{n-1}, x_{n}-a_{n}\right) .
$$


Logo,

$$
\begin{gathered}
\|A(x)-A(a)\| \leq\left\|A\left(x_{1}-a_{1}, x_{2}, \cdots, x_{n}\right)\right\|+\cdots+\left\|A\left(a_{1}, \cdots, a_{n-1}, x_{n}-a_{n}\right)\right\| \leq \\
\leq M\left\|x_{1}-a_{1}\right\|\left\|x_{2}\right\| \cdots\left\|x_{n}\right\|+\cdots+M\left\|a_{1}\right\| \cdots\left\|a_{n-1}\right\|\left\|x_{n}-a_{n}\right\| .
\end{gathered}
$$

Assim, se $\|x\|,\|a\|<R$, temos, para cada $1 \leq i \leq n,\left\|x_{i}\right\|,\left\|a_{i}\right\|<R$. Portanto:

$$
\|A(x)-A(a)\| \leq M R^{n-1}\left(\left\|x_{1}-a_{1}\right\|+\cdots+\left\|x_{n}-a_{n}\right\|\right) \leq M R^{n-1}\|x-a\|_{1} .
$$

Denotaremos por $L\left(E_{1}, \cdots, E_{n} ; F\right)$ o espaço de todas as aplicações $n$-lineares contínuas de $E_{1}, E_{2}, \cdots, E_{n}$ em $F$. Quando $F=\mathbb{K}$, escrevemos simplesmente $L\left(E_{1}, \cdots, E_{n}\right)$. No caso em que $E_{1}=E_{2}=\cdots=E_{n}=E$, escrevemos $L\left({ }^{n} E ; F\right)$. Por convenção, $L\left({ }^{0} E ; F\right)=F$.

A proposição seguinte relaciona a continuidade de uma aplicação multilinear com a continuidade em cada uma de suas variáveis. Deve-se notar que a hipótese sobre a completude dos espaços é essencial, como mostrará o exemplo após a proposição.

Proposição 4.3. Sejam $E_{1}, E_{2}, \cdots, E_{n}$ espaços de Banach e $F$ um espaço normado. Então, $A \in$ $L\left(E_{1}, \cdots, E_{n} ; F\right) \Leftrightarrow A$ é contínua em cada variável.

Demonstração. Se $A$ é contínua, então $A$ é claramente contínua em cada variável, pois essas funções são apenas restrições da multilinear $A$.

Reciprocamente, suponhamos que $A: E_{1} \times E_{2} \longrightarrow F$ seja bilinear e contínua em cada uma de suas variáveis. Em particular, a função $A_{y}: E_{1} \rightarrow F$ definida por $A_{y}(x)=A(x, y)$ é linear e contínua. Analogamente, pode-se definir a função $A_{x}: E_{2} \longrightarrow F$. Portanto:

$$
\|A(x, y)\|=\left\|A_{x}(y)\right\| \leq M_{x}\|y\|, \forall y \in E_{2} .
$$

Se $\|y\| \leq 1$, segue que $\left\|A_{y}(x)\right\|=\left\|A_{x}(y)\right\| \leq M_{x}$. Considere a família $\mathcal{F}=\left\{A_{y}:\|y\| \leq 1\right\}$. Então, $\left\|A_{y}(x)\right\| \leq M_{x}, \forall A_{y} \in \mathcal{F}$.

Pelo Teorema de Banach-Steinhauss (teorema 1.11), existe uma constante $M>0$ com $\left\|A_{y}\right\| \leq$ $M, \forall A_{y} \in \mathcal{F}$. Então, se $x \in E_{1},\|x\| \leq 1, y \in E_{2}$ e $\|y\| \leq 1$ :

$$
\|A(x, y)\|=\left\|A_{y}(x)\right\| \leq\left\|A_{y}\right\| \leq M
$$

e, conseqüentemente, para todo $(x, y) \in E_{1} \times E_{2}$, tem-se $\|A(x, y)\| \leq M\|x\|\|y\|$. Pela proposição 4.2, $A$ é contínua. 
Suponhamos, agora, que toda aplicação $(n-1)$-linear contínua em cada variável é contínua, e seja $A$ uma aplicação $n$-linear contínua em cada variável. Fixemos $x_{n} \in E_{n}$, e definamos:

$$
A_{x_{n}}\left(x_{1}, x_{2}, \cdots, x_{n-1}\right)=A\left(x_{1}, x_{2}, \cdots, x_{n-1}, x_{n}\right) .
$$

Então, $A_{x_{n}}$ é $(n-1)$-linear e contínua em cada variável. Logo, $A_{x_{n}}$ é contínua, donde existe $M_{x_{n}}$ tal que:

$$
\left\|A_{x_{n}}\left(x_{1}, x_{2}, \cdots, x_{n-1}\right)\right\|=\left\|A\left(x_{1}, x_{2}, \cdots, x_{n-1}, x_{n}\right)\right\| \leq M_{x_{n}}\left\|x_{1}\right\|\left\|x_{2}\right\| \cdots\left\|x_{n}\right\| .
$$

Se $\left\|x_{i}\right\| \leq 1,1 \leq i \leq n-1$, então $\left\|A\left(x_{1}, x_{2}, \cdots, x_{n-1}, x_{n}\right)\right\| \leq M_{x_{n}}$. Se definirmos

$$
A_{x_{1}, \cdots, x_{n-1}}\left(x_{n}\right)=A\left(x_{1}, x_{2}, \cdots, x_{n-1}, x_{n}\right)
$$

e a família $\mathcal{F}=\left\{A_{x_{1}, \cdots, x_{n-1}}:\left\|x_{i}\right\| \leq 1,1 \leq i \leq n-1\right\}$, teremos, para cada $x_{n} \in E_{n}$ :

$$
\left\|A_{x_{1}, \cdots, x_{n-1}}\left(x_{n}\right)\right\|=\left\|A\left(x_{1}, x_{2}, \cdots, x_{n-1}, x_{n}\right)\right\| \leq M_{x_{n}} .
$$

Utilizando novamente o Teorema de Banach-Steinhauss, existe uma constante positiva $M$ tal que $\left\|A_{x_{1}, \cdots, x_{n-1}}\right\| \leq M, \forall A_{x_{1}, \cdots, x_{n-1}} \in \mathcal{F}$. Analogamente, concluímos que $A$ é contínua.

Corolário 4.4. Se A é uma aplicação multilinear definida em um produto de espaços de dimensão finita, então $A$ é contínua.

Demonstração. Basta notar que todo espaço de dimensão finita é completo e que, além disso, toda função linear definida em um espaço de dimensão finita é contínua.

O exemplo seguinte mostra que a completude do espaço é essencial para que a proposição seja verdadeira.

Exemplo 4.5. Seja $E=C([0,1] ; \mathbb{R})$ munido da norma $\|x\|=\int_{0}^{1}|x(t)| d t$. A aplicação $f \in L_{a}\left({ }^{2} E\right)$, definida por $f(x, y)=\int_{0}^{1} x(t) y(t) d t$ é contínua em cada variável, mas não é contínua.

De fato, fixado $x \in E$, temos:

$$
|f(x, y)| \leq \int_{0}^{1}\left|x(t)\left\|y(t)\left|d t \leq\|x\|_{\infty} \int_{0}^{1}\right| y(t) \mid d t=\right\| x\left\|_{\infty}\right\| y \| .\right.
$$

Logo, a função é contínua na segunda variável, e o mesmo pode ser feito para mostrar que também é contínua na primeira variável. Vamos, agora, mostrar que $f$ não é contínua. Considere a seqüência em $C([0,1] ; \mathbb{R})$ definida por:

$$
x_{n}(t)=\left\{\begin{array}{cl}
n-n^{3} t & , 0 \leq t \leq \frac{1}{n^{2}} \\
0 & , \frac{1}{n^{2}}<t<1
\end{array}\right.
$$


Temos que:

$$
\left\|x_{n}\right\|=\int_{0}^{\frac{1}{n^{2}}}\left|n-n^{3} t\right| d t=\int_{0}^{\frac{1}{n^{2}}} n-n^{3} t d t=\frac{1}{2 n} .
$$

Logo, a seqüência $\left(\left(x_{n}, x_{n}\right)\right)_{n \in \mathbb{N}}$ converge a zero. No entanto:

$$
f\left(x_{n}, x_{n}\right)=\int_{0}^{\frac{1}{n^{2}}}\left(n-n^{3} t\right)^{2} d t=\frac{1}{3} .
$$

e $f$ não pode ser contínua.

A próxima proposição apresenta a norma utilizada no espaço das aplicações multilineares:

Proposição 4.6. Sejam $n \in \mathbb{N}, E_{1}, E_{2}, \cdots, E_{n}$ e $F$ espaços normados sobre o corpo $\mathbb{K}$. A função $\|\cdot\|: L\left(E_{1}, E_{2}, \cdots, E_{n} ; F\right) \longrightarrow \mathbb{R}$ dada por:

$$
\|A\|=\sup \left\{\|A(x)\|: x \in E_{1} \times E_{2} \times \cdots \times E_{n},\|x\| \leq 1\right\}
$$

é uma norma em $L\left(E_{1}, E_{2}, \cdots, E_{n} ; F\right)$.

Demonstração. Note que $\|A\| \in \mathbb{R}$, uma vez que $A$ é contínua e, portanto, vale a proposição 4.2 . Vamos demonstrar cada uma das propriedades da norma:

i) $\|A\|=0 \Leftrightarrow A=0$.

Imediato, a partir da definição.

ii) $\|\lambda A\|=|\lambda|\|A\|, \forall \lambda \in \mathbb{K}$.

Seja $\lambda \in \mathbb{K}$. Então:

$$
\|\lambda A\|=\sup \{\|\lambda A(x)\|:\|x\| \leq 1\}=|\lambda| \sup \{\|A(x)\|:\|x\| \leq 1\}=|\lambda|\|A\| .
$$

iii) $\left\|A_{1}+A_{2}\right\| \leq\left\|A_{1}\right\|+\left\|A_{2}\right\|$.

Sejam $A_{1}, A_{2} \in L\left(E_{1}, E_{2}, \cdots, E_{n} ; F\right)$ e $x \in E_{1} \times E_{2} \times \cdots \times E_{n}$ com $\|x\| \leq 1$. Então:

$$
\left\|A_{1}(x)+A_{2}(x)\right\| \leq\left\|A_{1}(x)\right\|+\left\|A_{2}(x)\right\| \leq\left\|A_{1}\right\|+\left\|A_{2}\right\| .
$$

Logo:

$$
\left\|A_{1}+A_{2}\right\|=\sup \left\{\left\|A_{1}(x)+A_{2}(x)\right\|:\|x\| \leq 1\right\} \leq\left\|A_{1}\right\|+\left\|A_{2}\right\|
$$

O próxima lema identifica o espaço das aplicações multilineares contínuas com um espaço de aplicações lineares, e serve como base para o resultado seguinte, que é bastante relevante. 
Lema 4.7. Sejam $E_{i}, F_{j}, G$ espaços normados, com $1 \leq i \leq m$ e $1 \leq j \leq n$. A função

$$
\Phi: L\left(E_{1}, \cdots, E_{m}, F_{1}, \cdots, F_{n} ; G\right) \longrightarrow L\left(E_{1}, \cdots, E_{m} ; L\left(F_{1}, \cdots, F_{n} ; G\right)\right)
$$

definida por

$$
\Phi(A)(x)(y)=A(x, y), \text { para } x \in E_{1} \times \cdots \times E_{m} \text { e } y \in F_{1} \times \cdots \times F_{n}
$$

é uma isometria.

Demonstração. É fácil ver que $\Phi$ é linear. A sobrejetividade também é clara: se $B \in L\left(E_{1}, \cdots, E_{m} ; L\left(F_{1}, \cdots, F_{n} ; G\right)\right)$, basta tomar $A(x, y)=B(x)(y)$. Assim, $A$ é multilinear e $A$ é contínua, pois:

$$
\|A(x, y)\|=\|B(x)(y)\| \leq\|B(x)\|\left\|y_{1}\right\| \cdots\left\|y_{n}\right\| \leq\|B\|\left\|x_{1}\right\| \cdots\left\|x_{m}\right\|\left\|y_{1}\right\| \cdots\left\|y_{n}\right\|
$$

para $x=\left(x_{1}, \cdots, x_{m}\right), y=\left(y_{1}, \cdots, y_{n}\right)$ e $\Phi(A)=B$. Vejamos, agora, que $\Phi$ é uma isometria e, conseqüentemente, é contínua. Dados $A \in L\left(E_{1}, \cdots, E_{m}, F_{1}, \cdots, F_{n} ; G\right), x \in E_{1} \times \cdots \times E_{m} \mathrm{e}$ $y \in F_{1} \times \cdots \times F_{m}, \operatorname{com}\|x\| \leq 1 \mathrm{e}\|y\| \leq 1$, temos:

$$
\|\Phi(A)\|=\sup _{\|x\|,\|y\| \leq 1}\|\Phi(A)(x)(y)\|=\sup _{\|x\|,\|y\| \leq 1}\|A(x)(y)\|=\|A\| .
$$

Como conseqüência direta desse lema, temos o seguinte:

Teorema 4.8. Sejam $n \in \mathbb{N}$ e $F$ um espaço de Banach. Então, $L\left(E_{1}, \cdots, E_{n} ; F\right)$ é um espaço de Banach, para quaisquer espaços normados $E_{1}, \cdots, E_{n}$.

Demonstração. Basta mostrarmos que o espaço é completo. Procedamos por indução. Se $n=1$, então estamos no caso linear, e o resultado já é conhecido.

Suponha que todo espaço de aplicações $n$-lineares seja completo, e seja $L\left(E_{0}, E_{1}, \cdots, E_{n} ; F\right)$ um espaço de aplicações $(n+1)$-lineares. Por hipótese, $L\left(E_{1}, \cdots, E_{n} ; F\right)$ é completo. Logo, $L\left(E_{0} ; L\left(E_{1}, \cdots, E_{n} ; F\right)\right)$ é completo, pois é um espaço de aplicações lineares em um contra-domínio completo.

Mas, pelo lema $4.7, L\left(E_{0}, E_{1}, \cdots, E_{n} ; F\right)$ é isométrico a $L\left(E_{0} ; L\left(E_{1}, \cdots, E_{n} ; F\right)\right)$. Como isometrias preservam completude, segue o resultado. 


\subsection{Aplicações Multilineares Simétricas}

Nessa seção, vamos restringir-nos às aplicações multilineares simétricas, em função do objetivo de estudar os polinômios homogêneos, que têm sua origem em aplicações desse tipo.

Para cada $n \in \mathbb{N}, \mathcal{S}_{n}$ denotará o conjunto de todas as permutações de $\{1,2, \cdots, n\}$. Dada $A \in L\left({ }^{n} E ; F\right)$, dizemos que $A$ é simétrica se $A\left(x_{1}, \cdots, x_{n}\right)=A\left(x_{\sigma(1)}, \cdots, x_{\sigma(n)}\right)$, para toda permutação $\sigma$ de $\mathcal{S}_{n}$.

Denotaremos por $L_{a s}\left({ }^{n} E ; F\right)$ o espaço das aplicações $n$-lineares simétricas de $E^{n}$ em $F$, e por $L_{s}\left({ }^{n} E ; F\right)$ o espaço das aplicações $n$-lineares simétricas e contínuas de $E^{n}$ em $F$. Quando $F=\mathbb{K}$, escrevemos simplesmente $L_{s}\left({ }^{n} E\right)$.

A fim de facilitar a notação utilizada ao longo do texto, vamos introduzir duas novas notações: para cada $k \in \mathbb{N}$ e para cada $k$-upla de naturais $\gamma=\left(n_{1}, \cdots, n_{k}\right)$, escrevemos:

$$
|\gamma|=\sum_{i=1}^{k} n_{k} \quad \text { e } \quad \gamma !=n_{1} ! \cdots n_{k} !
$$

Definição 4.9. Sejam $E$ e $F$ espaços normados sobre $\mathbb{K}, A \in L_{a}\left({ }^{n} E ; F\right)$ e $k \in \mathbb{N}$. Para cada $\left(x_{1}, \cdots, x_{k}\right) \in E^{k}$ e para cada $k$-upla de naturais $\left(n_{1}, \cdots, n_{k}\right)$ com $n_{1}+\cdots+n_{k}=n>0$, definimos:

$$
A x_{1}^{n_{1}} \cdots x_{k}^{n_{k}}=A(\overbrace{x_{1}, \cdots, x_{1}}^{n_{1} \text { vezes }}, \cdots, \overbrace{x_{k}, \cdots, x_{k}}^{n_{k} \text { vezes }})
$$

Se $n=0$, então $A x_{1}^{n_{1}} \cdots x_{k}^{n_{k}}=A$, de acordo com a identificação que fizemos.

A próxima proposição é conhecida como Fórmula de Leibniz:

Proposição 4.10. Sejam $E, F$ espaços normados sobre $\mathbb{K}$ e $A \in L_{a s}\left({ }^{n} E ; F\right)$. Se $x_{1}, \cdots, x_{k} \in E$, temos:

$$
A\left(x_{1}+\cdots+x_{k}\right)^{n}=\sum_{|\gamma|=n} \frac{n !}{\gamma !} A x_{1}^{n_{1}} \cdots x_{k}^{n_{k}}
$$

onde a somatória ocorre sobre todas as $k$-uplas de naturais $\gamma=\left(n_{1}, \cdots, n_{k}\right)$ que satisfazem $|\gamma|=n$.

Demonstração. Se $n=0$ ou $n=1$, o resultado é trivial. Assumamos a fórmula válida para $n \in \mathbb{N}$, e seja $A \in L_{\text {as }}\left({ }^{n+1} E ; F\right)$. Então:

$$
A\left(x_{1}+\cdots+x_{k}\right)^{n+1}=A\left(x_{1}+\cdots+x_{k}\right)\left(x_{1}+\cdots+x_{k}\right)^{n} .
$$

Aplicando a hipótese sobre a aplicação $n$-linear simétrica $A\left(x_{1}+\cdots+x_{k}\right)$, temos:

$$
A\left(x_{1}+\cdots+x_{k}\right)^{n+1}=\sum_{|\gamma|=n} \frac{n !}{\gamma !} A\left(x_{1}+\cdots+x_{k}\right) x_{1}^{n_{1}} \cdots x_{k}^{n_{k}}=
$$




$$
=\sum_{|\gamma|=n} \frac{n !}{\gamma !} A x_{1}^{n_{1}+1} \cdots x_{k}^{n_{k}}+\sum_{|\gamma|=n} \frac{n !}{\gamma !} A x_{1}^{n_{1}} x_{2}^{n_{2}+1} \cdots x_{k}^{n_{k}}+\cdots+\sum_{|\gamma|=n} \frac{n !}{\gamma !} A x_{1}^{n_{1}} \cdots x_{k}^{n_{k}+1}
$$

Para cada $1 \leq i \leq k$, definamos $\beta^{[i]}=\left(\beta_{1}^{[i]}, \cdots, \beta_{k}^{[i]}\right)$ tal que:

$$
\beta_{j}^{[i]}=\left\{\begin{array}{cl}
n_{i}+1 & , j=i \\
n_{j} & , 1 \leq j \leq k, j \neq i
\end{array}\right.
$$

Assim:

$$
\begin{aligned}
& A\left(x_{1}+\cdots+x_{k}\right)^{n+1}=\sum_{\left\{\beta^{[i]}\right]=n+1} \frac{n !}{\left(\beta_{1}^{[1]}-1\right) ! \beta_{2}^{[1]} ! \cdots \beta_{k}^{[1]} !} A x_{1}^{\beta_{1}^{[1]}} x_{2}^{\beta_{2}^{[1]}} \cdots x_{k}^{\beta_{k}^{[1]}}+\cdots \\
& \cdots+\sum_{\left.\mid \beta^{[k]}\right]=n+1} \frac{n !}{\beta_{1}^{[k]} ! \beta_{2}^{[k]} ! \cdots\left(\beta_{k}^{[k]}-1\right) !} A x_{1}^{\beta_{1}^{[k]}} x_{2}^{\beta_{2}^{[k]}} \cdots x_{k}^{\beta_{k}^{[k]}} \\
& =\sum_{\substack{|\beta|=n+1 \\
\beta_{1} \geq 1}} \frac{n !}{\left(\beta_{1}-1\right) ! \beta_{2} ! \cdots \beta_{k} !} A x_{1}^{\beta_{1}} x_{2}^{\beta_{2}} \cdots x_{k}^{\beta_{k}}+\cdots+ \\
& \cdots+\sum_{\substack{|\beta|=n+1 \\
\beta_{1} \geq 1}} \frac{n !}{\beta_{1} ! \beta_{2} ! \cdots\left(\beta_{k}-1\right) !} A x_{1}^{\beta_{1}} x_{2}^{\beta_{2}} \cdots x_{k}^{\beta_{k}} \\
& =\sum_{\substack{|\beta|=n+1 \\
\beta_{1} \geq 1}} \frac{n ! \beta_{1}}{\beta_{1} ! \beta_{2} ! \cdots \beta_{k} !} A x_{1}^{\beta_{1}} x_{2}^{\beta_{2}} \cdots x_{k}^{\beta_{k}}+\cdots+\sum_{\substack{|\beta|=n+1 \\
\beta_{1} \geq 1}} \frac{n ! \beta_{k}}{\beta_{1} ! \beta_{2} ! \cdots \beta_{k} !} A x_{1}^{\beta_{1}} x_{2}^{\beta_{2}} \cdots x_{k}^{\beta_{k}}
\end{aligned}
$$

onde $\beta=\left(\beta_{1}, \cdots, \beta_{k}\right)$. Temos, portanto:

$$
\begin{gathered}
A\left(x_{1}+\cdots+x_{k}\right)^{n+1}=\sum_{|\beta|=n+1} n ! A x_{1}^{\beta_{1}} x_{2}^{\beta_{2}} \cdots x_{k}^{\beta_{k}}\left(\frac{\beta_{1}}{\beta_{1} ! \cdots \beta_{k} !}+\cdots+\frac{\beta_{k}}{\beta_{1} ! \cdots \beta_{k} !}\right) \\
=\sum_{|\beta|=n+1} \frac{n !}{\beta_{1} ! \cdots \beta_{k} !} A x_{1}^{\beta_{1}} x_{2}^{\beta_{2}} \cdots x_{k}^{\beta_{k}}\left(\beta_{1}+\cdots+\beta_{k}\right) \\
=\sum_{|\beta|=n+1} \frac{(n+1) !}{\beta !} A x_{1}^{\beta_{1}} x_{2}^{\beta_{2}} \cdots x_{k}^{\beta_{k}}
\end{gathered}
$$

que é exatamente a fórmula que procurávamos.

Corolário 4.11. Nesse contexto, se $k=2$, vale a seguinte fórmula binomial:

$$
A(x+y)^{n}=\sum_{i=0}^{n}\left(\begin{array}{c}
n \\
i
\end{array}\right) A x^{n-i} y^{i}
$$


Demonstração. Imediata, a partir da aplicação do resultado anterior.

O próximo teorema é conhecido como Fórmula de Polarização. Esse resultado é notável, pois mostra que uma aplicação multilinear simétrica pode ser totalmente determinada apenas pelos valores que assume na diagonal do espaço onde está definida.

Teorema 4.12. Sejam $E$ e $F$ espaços normados sobre $\mathbb{K}$. Se $A \in L_{a s}\left({ }^{n} E ; F\right)$, então:

$$
A\left(x_{1}, \cdots, x_{n}\right)=\frac{1}{n ! 2^{n}} \sum_{\substack{1 \leq i \leq n \\ \epsilon_{i}= \pm 1}} \epsilon_{1} \cdots \epsilon_{n} A\left(\epsilon_{1} x_{1}+\cdots+\epsilon_{n} x_{n}\right)^{n}
$$

para quaisquer $x_{1}, \cdots, x_{n} \in E$.

Demonstração. Pela proposição 4.10 , temos:

$$
\begin{gathered}
A\left(\epsilon_{1} x_{1}+\cdots+\epsilon_{n} x_{n}\right)^{n}=\sum_{\gamma} \frac{n !}{n_{1} ! \cdots n_{n} !} A\left(\epsilon_{1} x_{1}\right)^{n_{1}} \cdots\left(\epsilon_{n} x_{n}\right)^{n_{n}} \\
=\sum_{\gamma} \frac{n !}{n_{1} ! \cdots n_{n} !} \epsilon_{1}^{n_{1}} \cdots \epsilon_{n}^{n_{n}} A x_{1}^{n_{1}} \cdots x_{n}^{n_{n}}
\end{gathered}
$$

onde $\gamma=\left(n_{1}, \cdots, n_{n}\right) \in \mathbb{N}^{n}$ e $|\gamma|=n$. Portanto:

$$
\begin{gathered}
\sum_{\substack{1 \leq i \leq n \\
\epsilon_{i}= \pm 1}} \epsilon_{1} \cdots \epsilon_{n} A\left(\epsilon_{1} x_{1}+\cdots+\epsilon_{n} x_{n}\right)^{n}= \\
=\sum_{\substack{1 \leq i \leq n \\
\epsilon_{i}= \pm 1}} \epsilon_{1} \cdots \epsilon_{n} \sum_{\gamma} \frac{n !}{n_{1} ! \cdots n_{n} !} \epsilon_{1}^{n_{1}} \cdots \epsilon_{n}^{n_{n}} A x_{1}^{n_{1}} \cdots x_{n}^{n_{n}} \\
=\sum_{\gamma} \frac{n !}{n_{1} ! \cdots n_{n} !} A x_{1}^{n_{1}} \cdots x_{n}^{n_{n}} \sum_{\substack{1 \leq i \leq n \\
\epsilon_{i}= \pm 1}} \epsilon_{1}^{n_{1}+1} \cdots \epsilon_{n}^{n_{n}+1} .
\end{gathered}
$$

Se $n_{j}=0$, para algum $1 \leq j \leq n$, temos $\epsilon^{n_{j}+1}=\epsilon= \pm 1$. Logo:

$$
\sum_{\substack{1 \leq i \leq n \\ \epsilon_{i}= \pm 1}} \epsilon_{1}^{n_{1}+1} \cdots \epsilon_{n}^{n_{n}+1}=\sum_{\substack{i \neq j \\ \epsilon_{i}= \pm 1}} \epsilon_{1}^{n_{1}+1} \cdots \epsilon_{n}^{n_{n}+1}-\sum_{\substack{i \neq j \\ \epsilon_{i}= \pm 1}} \epsilon_{1}^{n_{1}+1} \cdots \epsilon_{n}^{n_{n}+1}=0 .
$$

Dessa forma, as únicas parcelas que contribuem para a soma são aquelas em que cada $n_{i}$ não é nulo. Mas então devemos ter $n_{1}=n_{2}=\cdots=n_{n}=1$. Assim, a soma consiste de uma única parcela. Nesse caso,

$$
\sum_{\substack{1 \leq i \leq n \\ \epsilon_{i}= \pm 1}} \epsilon_{1}^{2} \cdots \epsilon_{n}^{2}=2^{n}
$$


Então:

$$
\sum_{\substack{1 \leq i \leq n \\ \epsilon_{i}= \pm 1}} \epsilon_{1} \cdots \epsilon_{n} A\left(\epsilon_{1} x_{1}+\cdots+\epsilon_{n} x_{n}\right)^{n}=n ! 2^{n} A\left(x_{1}, \cdots, x_{n}\right)
$$

e segue o resultado.

\subsection{Polinômios}

Nessa seção, definiremos os conceitos de polinômios homogêneos e de polinômios. Apresentaremos, também, alguns teoremas sobre sua continuidade. Como vamos trabalhar apenas com polinômios contínuos em espaços de Banach, as definições seguintes se restringirão a espaços normados. No entanto, cabe ressaltar que os polinômios podem ser definidos em espaços vetoriais arbitrários.

Definição 4.13. Sejam $E$ e $F$ espaços normados sobre o corpo $\mathbb{K}$. Dizemos que uma função $P: E \longrightarrow F$ é um polinômio $n$-homogêneo se existe $A: E^{n} \longrightarrow F$ aplicação $n$-linear tal que $P(x)=A x^{n}, \forall x \in E$.

Nesse caso, dizemos que $P$ é o polinômio $n$-homogêneo associado a $A$. Denotaremos por $\mathcal{P}_{a}\left({ }^{n} E ; F\right)$ o espaço vetorial de todos os polinômios n-homogêneos de $E$ em $F$. Quando $F=\mathbb{K}$, escrevemos simplesmente $\mathcal{P}_{a}\left({ }^{n} E\right)$. Além disso, identificaremos $\mathcal{P}_{a}\left({ }^{0} E ; F\right)$ por $F$.

A seguir, definiremos um polinômio no espaço $E$.

Definição 4.14. Sejam $E$ e $F$ espaços normados sobre o corpo $\mathbb{K}$. Dizemos que uma função $P: E \longrightarrow F$ é um polinômio se pode ser representada como uma soma:

$$
P=P_{0}+P_{1}+\cdots+P_{m}
$$

onde $P_{i} \in \mathcal{P}_{a}\left({ }^{i} E ; F\right), i=0, \cdots, m$. Se $P_{m} \not \equiv 0$, diz-se que $P$ é um polinômio de grau $m$.

O espaço vetorial de todos os polinômios de $E$ em $F$ será denotado por $\mathcal{P}_{a}(E ; F)$. Se $F=\mathbb{K}$, escreveremos apenas $\mathcal{P}_{a}(E)$.

A próxima proposição analisa as aplicações multilineares associadas a um polinômio homogêneo:

Proposição 4.15. Sejam $E, F$ espaços normados sobre $\mathbb{K} e n \in \mathbb{N}$. Se $P \in \mathcal{P}_{a}\left({ }^{n} E ; F\right)$, existe uma única aplicação n-linear simétrica $A$ tal que $P(x)=A x^{n}, \forall x \in E$. 
Demonstração. Por definição, se $P \in \mathcal{P}_{a}\left({ }^{n} E ; F\right)$, então existe uma aplicação $n$-linear $A$ tal que $P(x)=A x^{n}, \forall x \in E$. Essa aplicação não é, necessariamente, simétrica. Definamos:

$$
A_{s}\left(x_{1}, \cdots, x_{n}\right)=\frac{1}{n !} \sum_{\sigma \in \mathcal{S}_{n}} A\left(x_{\sigma(1)}, \cdots, x_{\sigma(n)}\right) .
$$

É claro que $A_{s} x^{n}=A x^{n}=P(x)$, e provamos a existência. A unicidade da aplicação é conseqüência imediata do teorema 4.12, uma vez que duas aplicaçôes multilineares simétricas que coincidem na diagonal devem coincidir em todo o domínio.

A seguir, mostraremos que a representação de um polinômio como soma de polinômios homogêneos é única:

Proposição 4.16. Sejam $E$ e $F$ espaços normados sobre $\mathbb{K}$. $O$ espaço vetorial $\mathcal{P}_{a}(E ; F)$ é a soma direta algébrica dos espaços $\mathcal{P}_{a}\left({ }^{n} E ; F\right)$, com $n \in \mathbb{N}$.

Demonstração. Por definição, todo polinômio pode ser representado como soma finita de polinômios homogêneos. Resta, então, mostrar que $P=P_{0}+\cdots+P_{n}=0 \Rightarrow P_{0}=P_{1}=\cdots=P_{n}=0$.

Sejam $x \in E$ e $\lambda \neq 0$. Então:

$$
\begin{gathered}
P_{0}(\lambda x)+P_{1}(\lambda x)+\cdots+P_{n}(\lambda x)=0 \Rightarrow P_{0}(x)+\lambda P_{1}(x)+\cdots+\lambda^{n} P_{n}(x)=0 \\
\Rightarrow \frac{1}{\lambda^{n}} P_{0}(x)+\frac{1}{\lambda^{n-1}} P_{1}(x)+\cdots+P_{n}(x)=0 .
\end{gathered}
$$

Logo, fazendo $\lambda \rightarrow \infty$, temos que $P_{n}(x)=0$. Utilizando raciocínio análogo, e lembrando que $x \in E$ é arbitrário, segue que $P_{0}=P_{1}=\cdots=P_{n}=0$.

A próxima proposição apresenta algumas equivalências para a continuidade de um polinômio n-homogêneo:

Proposição 4.17. Sejam $E, F$ espaços normados sobre $\mathbb{K}, n \in \mathbb{N}, P \in \mathcal{P}_{a}\left({ }^{n} E ; F\right)$ e $A \in L_{a s}\left({ }^{n} E ; F\right)$, com $P$ associado a $A$. São equivalentes:

1. $A \in L_{s}\left({ }^{n} E ; F\right)$;

2. Pé contínuo;

3. P é contínuo na origem;

4. Existe $M>0$ tal que $\|P(x)\| \leq M\|x\|^{n}$, para qualquer $x \in E^{n}$. 
Demonstração. (1) $\Rightarrow(2)$ : Evidente, pois $P=A \circ i$, onde $i: E \longrightarrow E^{n}$ é a inclusão.

(2) $\Rightarrow(3)$ : Óbvio.

(3) $\Rightarrow(4)$ : Por hipótese, $P$ é contímuo em 0 . Além disso, $P(0)=0$. Assim, dado $\epsilon=1$, existe $\delta>0$ tal que, se $\|x\|<\delta$, então $\|P(x)\| \leq 1$. Dado $x \in E$, com $x \neq 0$, temos:

$$
1 \geq\left\|P\left(\frac{\delta x}{2\|x\|}\right)\right\|=\left\|A\left(\frac{\delta x}{2\|x\|}\right)^{n}\right\|=\frac{\delta^{n}\left\|A x^{n}\right\|}{2^{n}\|x\|^{n}} .
$$

Logo,

$$
\|P(x)\| \leq \frac{2^{n}}{\delta^{n}}\|x\|^{n}, \quad \forall x \in E, x \neq 0 .
$$

Como a desigualdade permanece válida para $x=0$, segue o resultado.

$(4) \Rightarrow(1)$ : Sejam $x_{1}, \cdots, x_{n} \in E$ tais que $\left\|x_{i}\right\| \leq \frac{1}{n}$, para $i=1, \cdots, n$. Pelo teorema 4.12:

$$
\begin{gathered}
\left\|A\left(x_{1}, \cdots, x_{n}\right)\right\| \leq \frac{1}{n ! 2^{n}} \sum_{\substack{1 \leq i \leq n \\
\epsilon_{i}= \pm 1}}\left|\epsilon_{1}\right| \cdots\left|\epsilon_{n}\right|\left\|A\left(\epsilon_{1} x_{1}+\cdots+\epsilon_{n} x_{n}\right)^{n}\right\| \\
=\frac{1}{n ! 2^{n}} \sum_{\substack{1 \leq i \leq n \\
\epsilon_{i}= \pm 1}}\left\|P\left(\epsilon_{1} x_{1}+\cdots+\epsilon_{n} x_{n}\right)\right\| \leq \frac{1}{n ! 2^{n}} \sum_{\substack{1 \leq i \leq n \\
\epsilon_{i}= \pm 1}} M\left\|\left(\epsilon_{1} x_{1}+\cdots+\epsilon_{n} x_{n}\right)^{n}\right\| \\
\leq \frac{1}{n ! 2^{n}} \sum_{\substack{1 \leq i \leq n \\
\epsilon_{i}= \pm 1}} M\left(\left\|x_{1}+\cdots+\right\| x_{n} \|\right)^{n} \leq \frac{1}{n ! 2^{n}} \sum_{\substack{1 \leq i \leq n \\
\epsilon_{i}= \pm 1}} M=\frac{1}{n ! 2^{n}} 2^{n} M=\frac{M}{n !} .
\end{gathered}
$$

Mas, para quaisquer $x_{1}, \cdots, x_{n} \in E, \operatorname{com} x_{i} \neq 0$, podemos definir $y_{i}=\frac{x_{i}}{n\left\|x_{i}\right\|}$. Então, $\left\|y_{i}\right\| \leq \frac{1}{n !}$, e:

$$
\left\|A\left(y_{1}, \cdots, y_{n}\right)\right\|=\frac{\left\|A\left(x_{1}, \cdots, x_{n}\right)\right\|}{n^{n}\left\|x_{1}\right\| \cdots\left\|x_{n}\right\|} \leq \frac{M}{n !} .
$$

Logo:

$$
\left\|A\left(x_{1}, \cdots, x_{n}\right)\right\| \leq \frac{M n^{n}}{n !}\left\|x_{1}\right\| \cdots\left\|x_{n}\right\|, \quad \forall x \in E, x \neq 0 .
$$

Como a desigualdade permanece válida para $x=0$, segue que $A \in L_{s}\left({ }^{n} E ; F\right)$.

Denotaremos por $\mathcal{P}\left({ }^{n} E ; F\right)$ o espaço vetorial dos polinômios $n$-homogêneos contínuos de $E$ em $F$. Quando $F=\mathbb{K}$, escrevemos simplesmente $\mathcal{P}\left({ }^{n} E\right)$. O espaço vetorial dos polinômios contínuos de $E$ em $F$ será denotado por $\mathcal{P}(E ; F)$.

A proposição seguinte garante que o espaço dos polinômios $n$-homogêneos é um espaço normado: 
Proposição 4.18. Sejam $E, F$ espaços normados sobre $\mathbb{K}$ e $P \in \mathcal{P}\left({ }^{n} E ; F\right)$. Então,

$$
\|P\|=\sup _{\|x\|=1}|P(x)|
$$

é uma norma em $\mathcal{P}\left({ }^{n} E ; F\right)$.

Demonstração. Análoga à proposição 4.6 .

A proposição seguinte estabelece uma relação entre a norma de um polinômio homogêneo e a norma da aplicação multilinear associada a ele:

Proposição 4.19. A função $\Psi: L_{s}\left({ }^{n} E ; F\right) \longrightarrow \mathcal{P}\left({ }^{n} E ; F\right)$ dada por $\Psi(A)=P$, que associa cada multilinear simétrica a um polinômio homogêneo, é um isomorfismo de espaços normados. Além disso, vale a seguinte desigualdade:

$$
\|P\| \leq\|A\| \leq \frac{n^{n}}{n !}\|P\|
$$

Demonstração. O isomorfismo é imediato. Falta, então, mostramos a desigualdade. Se $n=0$ ou $n=1$, então $A=P$, e a desigualdade é trivialmente verificada. Suponhamos, então, $n \geq 2$.

Como $\|P(x)\|=\left\|A x^{n}\right\| \leq\|A\|\|x\|^{n}$, segue que $\|P\| \leq\|A\|$. Por outro lado, fixe $x=$ $\left(x_{1}, \cdots, x_{n}\right) \in E^{n}$ com $\|x\| \leq 1$. Temos:

$$
\begin{gathered}
\left\|A\left(x_{1}, \cdots, x_{n}\right)\right\| \leq \frac{1}{n ! 2^{n}} \sum_{\substack{1 \leq i \leq n \\
\epsilon_{i}= \pm 1}}\left\|P\left(\epsilon_{1} x_{1}+\cdots+\epsilon_{n} x_{n}\right)\right\| \\
\leq \frac{1}{n ! 2^{n}} \sum_{\substack{1 \leq i \leq n \\
\epsilon_{i}= \pm 1}}\|P\|\left\|\epsilon_{1} x_{1}+\cdots+\epsilon_{n} x_{n}\right\|^{n} \leq \frac{1}{n ! 2^{n}} \sum_{\substack{1 \leq i \leq n \\
\epsilon_{i}= \pm 1}}\|P\|\left(\left\|x_{1}\right\|+\cdots+\left\|x_{n}\right\|\right)^{n} \\
=\frac{1}{n !}\|P\|\left(\left\|x_{1}\right\|+\cdots+\left\|x_{n}\right\|\right)^{n} \leq \frac{1}{n !}\|P\| n^{n}
\end{gathered}
$$

Logo, $\|A\| \leq \frac{n^{n}}{n !}\|P\|$.

A corolário a seguir nos diz que $\mathcal{P}\left({ }^{n} E ; F\right)$ é um espaço de Banach, quando $F$ o é.

Corolário 4.20. Sejam $E$ um espaço normado e $F$ um espaço de Banach sobre $\mathbb{K}$. Então, para cada $n \in \mathbb{N}, \mathcal{P}\left({ }^{n} E ; F\right)$ é um espaço de Banach.

Demonstração. Se $n=0$, temos $\mathcal{P}\left({ }^{0} E ; F\right)=F$, e $F$ é, por hipótese, um espaço de Banach. Se $n=1$, então estamos no caso linear, para o qual já sabemos que tal resultado é verdadeiro.

Suponhamos, então, $n \geq 2$. Em virtude da proposição 4.19 , é preciso apenas mostrar que $L_{s}\left({ }^{n} E ; F\right) \subseteq L\left({ }^{n} E ; F\right)$ é fechado, uma vez que, pelo teorema $4.8, L\left({ }^{n} E ; F\right)$ é completo. 
Se $A \in \overline{L_{s}\left({ }^{n} E ; F\right)}$, então existe uma seqüência $\left(A_{n}\right)_{n \in \mathbb{N}}$ em $L_{s}\left({ }^{n} E ; F\right)$ tal que $A_{n} \stackrel{\|\cdot\|}{\longrightarrow} A$. Fixado $x=\left(x_{1}, \cdots, x_{n}\right) \in E^{n}$, temos:

$$
\left\|A_{n}\left(x_{1}, \cdots, x_{n}\right)-A\left(x_{1}, \cdots, x_{n}\right)\right\| \leq\left\|A_{n}-A\right\|\left\|x_{1}\right\| \cdots\left\|x_{n}\right\| .
$$

Então, $A_{n}\left(x_{1}, \cdots, x_{n}\right) \longrightarrow A\left(x_{1}, \cdots, x_{n}\right)$, para todo $x=\left(x_{1}, \cdots, x_{n}\right) \in E^{n}$. Mas, para toda permutação $\sigma$ de $\mathcal{S}_{n}$, temos:

$$
A_{n}\left(x_{1}, \cdots, x_{n}\right)=A_{n}\left(x_{\sigma(1)}, \cdots, x_{\sigma(n)}\right) \rightarrow A\left(x_{\sigma(1)}, \cdots, x_{\sigma(n)}\right) .
$$

Logo, $A\left(x_{1}, \cdots, x_{n}\right)=A\left(x_{\sigma(1)}, \cdots, x_{\sigma(n)}\right)$, para toda permutação $\sigma$ de $\mathcal{S}_{n}$, donde $A \in L_{s}\left({ }^{n} E ; F\right)$, e segue o resultado.

A próxima proposição mostra que, também no caso de polinômios, continuidade implica continuidade uniforme sobre os limitados. Isso já ocorria para aplicações lineares.

Proposição 4.21. Sejam $E, F$ espaços normados sobre $\mathbb{K}$ e $P \in \mathcal{P}\left({ }^{n} E ; F\right)$. Então, $P$ é uniformemente contínuo sobre os limitados de $E$.

Demonstração. Sejam $A \in L\left({ }^{n} E ; F\right)$ a multilinear associada a $P$ e $B \subseteq E$ limitado. Se $x, y \in B$, existe $R>0$ tal que $\|x\|,\|y\|<R$. Temos:

$$
\begin{gathered}
\|P(x)-P(y)\|=\left\|A x^{n}-A y^{n}\right\|=\| A(x, \cdots, x)-A(y, x, \cdots, x)+A(y, x, \cdots, x)- \\
-A(y, y, x, \cdots, x)+A(y, y, x, \cdots, x)-\cdots-A(y, \cdots, y, x)+A((y, \cdots, y, x)-A(y, \cdots, y) \| \\
\leq\|A(x-y, x, \cdots, x)\|+\|A(y, x-y, x, \cdots, x)\|+\cdots+\|A(y, \cdots, y, x-y)\| .
\end{gathered}
$$

Logo,

$$
\|P(x)-P(y)\| \leq n\|A\| R^{n-1}\|x-y\| .
$$

Tomando $M=n\|A\| R^{n-1}$, segue o resultado.

\subsection{Diferenciabilidade}

Nessa seção, estudaremos, de forma bastante superficial, a diferenciabilidade de polinômios em espaços de Banach. Apresentaremos a sua definição e alguns resultados básicos sobre ela.

Definição 4.22. Sejam $E, F$ espaços de Banach sobre $\mathbb{K}, U \subseteq E$ um subconjunto aberto em $E$ e $f: U \longrightarrow F$ uma função. Dizemos que $f$ é diferenciável no ponto $a \in U$ se existe uma aplicação linear $A: E \longrightarrow F$ tal que:

$$
\lim _{x \rightarrow a} \frac{\|f(x)-f(a)-A(x-a)\|}{\|x-a\|}=0
$$


Dizemos que $f$ é diferenciável se $f$ é diferenciável em cada ponto $a \in U$.

Observamos que, se $f$ é diferenciável, então $f$ é contínua, e que a função $A: E \longrightarrow F$ é unicamente determinada por $f$ e por $a \in U$. Dessa forma, chamaremos a função $A$ de diferencial de $f$ no ponto $a \in U$, e a denotaremos por $d f(a)$.

O exemplo seguinte apresenta o cálculo da diferencial de um polinômio:

Exemplo 4.23. Se $P \in \mathcal{P}\left({ }^{n} E\right)$ e $A$ é a aplicação $n$-linear associada a $P$, então:

$$
d P(a)=n A a^{n-1}, \forall a \in E .
$$

De fato, pela fórmula binomial, temos:

$$
P(x)=P(a)+n A a^{n-1}(x-a)+\sum_{i=2}^{n}\left(\begin{array}{l}
n \\
i
\end{array}\right) A a^{n-i}(x-a)^{i} .
$$

Seja $\phi(x)=P(x)-P(a)+n A a^{n-1}(x-a)$. Então,

$$
\phi(x)=\sum_{i=2}^{n}\left(\begin{array}{c}
n \\
i
\end{array}\right) A a^{n-i}(x-a)^{i}
$$

donde $\lim _{x \rightarrow a} \frac{|\phi(x)|}{\|x-a\|}=0$.

A proposição seguinte fornece a "regra da cadeia" para funções em um espaço de Banach:

Proposição 4.24. Sejam $E$ e $F$ espaços de Banach sobre $\mathbb{K}, U \subseteq E$ e $V \subseteq F$ dois subconjuntos abertos e $f: U \longrightarrow F$ eg $: V \longrightarrow \mathbb{K}$ funções diferenciáveis tais que $f(U) \subseteq V$. Então, gof $: U \longrightarrow \mathbb{K}$ é diferenciável ed $d(g \circ f)(a)=d g(f(a)) \circ d f(a), \forall a \in U$.

Demonstração. Sejam $a \in U$ e $b=f(a) \in V$. Definamos $A=d f(a)$ e $B=d g(b)$. Então, para cada $x \in U$ e cada $y \in V$, podemos escrever:

$$
f(x)=f(a)+A(x-a)+\phi(x) \quad \text { e } \quad g(y)=g(b)+B(y-b)+\psi(y)
$$

onde:

$$
\lim _{x \rightarrow a} \frac{\|\phi(x)\|}{\|x-a\|}=0 \quad \text { e } \quad \lim _{y \rightarrow b} \frac{\|\psi(y)\|}{\|y-b\|}=0
$$

Então:

$$
\begin{gathered}
g(f(x))=g(f(a))+B(f(x)-f(a))+\psi(f(x))=g(f(a))+B[A(x-a)+\psi(x)]+\psi(f(x))= \\
g(f(a))+B \circ A(x-a)+\rho(x)
\end{gathered}
$$


onde $\rho(x)=B(\phi(x))+\psi(f(x))$. Então:

$$
\frac{\|\rho(x)\|}{\|x-a\|} \leq\|B\| \frac{\|\phi(x)\|}{\|x-a\|}+\frac{\|\psi(f(x))\|}{\|f(x)-f(a)\|} \frac{\|f(x)-f(a)\|}{\|x-a\|}
$$

e, como

$$
\frac{\|f(x)-f(a)\|}{\|x-a\|}=\frac{\|A(x-a)+\phi(x)\|}{\|x-a\|} \leq\|A\|+\frac{\|\phi(x)\|}{\|x-a\|}
$$

segue que $\lim _{x \rightarrow a} \frac{\|\rho(x)\|}{\|x-a\|}=0$, o que completa a prova.

A seguir, vamos demonstrar uma generalização do conhecido Teorema do Valor Médio.

Teorema 4.25. Sejam $U \subseteq E$ um subconjunto aberto e $f: U \longrightarrow \mathbb{K}$ uma função diferenciável. Se o segmento $[a, a+t]=\{(1-\lambda) a+\lambda(a+t): \lambda \in[0,1]\} \subset U$, então:

$$
\|f(a+t)-f(a)\| \leq\|t\| \sup _{0 \leq \lambda \leq 1}\|d f(a+\lambda t)\| .
$$

Demonstração. Como a diferenciabilidade complexa implica a diferenciabilidade real, podemos assumir $\mathbb{K}=\mathbb{R}$. Considere a função $g:[0,1] \longrightarrow \mathbb{R}$ dada por:

$$
g(\lambda)=f(a+\lambda t)
$$

Então, $g$ é contínua em $[0,1]$, diferenciável em $(0,1)$ e $g^{\prime}(\lambda)=d f(a+\lambda t) t$ para cada $\lambda \in(0,1)$. Pelo Teorema do Valor Médio, segue que:

$$
|g(1)-g(0)| \leq \sup _{0 \leq \lambda \leq 1}\left|g^{\prime}(\lambda)\right|
$$

donde segue o resultado procurado.

Corolário 4.26. Sejam $U \subseteq E$ um subconjunto aberto e $f: U \longrightarrow \mathbb{K}$ uma função diferenciável. Se o segmento $[a, a+t] \subset U$, entào:

$$
\|f(a+t)-f(a)-d f(a)(t)\| \leq\|t\| \sup _{0 \leq \lambda \leq 1}\|d f(a+\lambda t)-d f(a)\| .
$$

Demonstração. Basta aplicar o teorema 4.25 à função $g: U \longrightarrow \mathbb{K}$ definida por:

$$
g(x)=f(x)-d f(a)(x-a)
$$




\section{Capítulo 5}

\section{Convergência de conjuntos de nível de polinômios}

Nesse capítulo, apresentaremos algumas caracterizações da convergência uniforme e da convergência pontual de polinômios homogêneos em espaços de Banach através da convergência de seus conjuntos de nível.

Apresentaremos, também, algumas generalizações para o caso de polinômios. Esse capítulo baseia-se no texto científico [8].

\subsection{Introdução}

Ao longo do presente capítulo, denotaremos por $E$ um espaço de Banach sobre o corpo $\mathbb{K}$. Se $P \in \mathcal{P}(E)$ e $\alpha \in \mathbb{K}$, o conjunto de nível $\alpha$ de $P$, assim como já definido para funcionais lineares contínuos, é dado por:

$$
L(P ; \alpha)=\{x \in E: P(x)=\alpha\}
$$

Exemplo 5.1. A seguir, apresentamos dois exemplos de conjuntos de nível de polinômios:

a) Considere $E=\mathbb{R}^{2}$, e $P(x, y)=x^{2}+y^{2}$. O conjunto de nível $\alpha>0$ de $P$ é:

$$
L(P ; \alpha)=\left\{(x, y) \in \mathbb{R}^{2}: x^{2}+y^{2}=\alpha\right\},
$$

ou seja, a circunferência de centro na origem e raio $\sqrt{\alpha}$.

b) Considere $E=l_{2}(\mathbb{R})$ e $P(x)=\sum_{i=1}^{\infty} x_{i}^{2}$. O conjunto de nível $\alpha>0$ de $P$ é:

$$
L(P ; \alpha)=\left\{x \in l_{2}(\mathbb{R}): \sum_{i=1}^{\infty} x_{i}^{2}=\alpha\right\},
$$


ou seja, a esfera em $l_{2}(\mathbb{R})$ de centro na origem e raio $\sqrt{\alpha}$.

Para denotar as convergências dos conjuntos de nível de uma sequiência de polinômios, utilizaremos as notações a seguir. Se $P \in \mathcal{P}(E)$ e $\left(P_{n}\right)_{n \in \mathbb{N}} \subset \mathcal{P}(E)$, dizemos que:

- $P_{n} \stackrel{K}{\longrightarrow} P$ se $\left(L\left(P_{n} ; \alpha\right)\right)_{n \in \mathbb{N}}$ é convergente, no sentido de Kuratowski, a $L(P ; \alpha), \forall \alpha \in \mathbb{K}$;

- $P_{n} \stackrel{\mathrm{W}}{\longrightarrow} P$ se $\left(L\left(P_{n} ; \alpha\right)\right)_{n \in \mathbb{N}}$ é convergente, no sentido de Wijsman, a $L(P ; \alpha), \forall \alpha \in \mathbb{K}$;

- $P_{n} \stackrel{\mathrm{r}}{\longrightarrow} P$ se $\left(L\left(P_{n} ; \alpha\right)\right)_{n \in \mathbb{N}}$ é r-convergente a $L(P ; \alpha), \forall \alpha \in \mathbb{K}$.

A mesma notação será utilizada no caso de polinômios homogêneos. A seguir, apresentaremos algumas observações que serão úteis ao longo do capítulo.

Observação 5.2. Consideremos $P \in \mathcal{P}\left({ }^{k} E\right)$ e $\left(P_{n}\right)_{n \in \mathbb{N}} \subset \mathcal{P}\left({ }^{k} E\right)$.

a) $L(P ; \alpha)=\alpha^{\frac{1}{k}} L(P ; 1), \forall \alpha \in \mathbb{C} \backslash\{0\}$.

De fato, se $x \in L(P ; \alpha)$, então $P(x)=\alpha$. Como $P$ é homogêneo, $P\left(\alpha^{\frac{-1}{k}} x\right)=1$, donde $x \in \alpha^{\frac{1}{k}} L(P ; 1)$. Reciprocamente, se $x \in \alpha^{\frac{1}{k}} L(P ; 1)$, então $x=\alpha^{\frac{1}{k}} y$, com $P(y)=1$. Logo, $P(x)=P\left(\alpha^{\frac{1}{k}} y\right)=\alpha P(y)=\alpha$, e $x \in L(P ; \alpha)$.

b) Seja $\theta=\mathrm{K}$, W ou r. Se $\alpha \in \mathbb{C} \backslash\{0\}$, temos:

$$
\left(L\left(P_{n} ; \alpha\right)\right)_{n \in \mathbb{N}} \text { é } \theta \text {-convergente a } L(P ; \alpha) \Leftrightarrow\left(L\left(P_{n} ; 1\right)\right)_{n \in \mathbb{N}} \text { é } \theta \text {-convergente a } L(P ; 1) \text {. }
$$

É importante notar que ambas as observações continuam válidas para o caso em que $\alpha \in \mathbb{R}$ e $k$ é ímpar. No entanto, caso tenhamos $k$ par, devemos supor $\alpha>0$.

c) Seja $\theta=\mathrm{K}, \mathrm{W}$ ou r. Temos que $P_{n} \stackrel{\theta}{\longrightarrow} P \Leftrightarrow\left(L\left(P_{n} ; 1\right)\right)_{n \in \mathbb{N}}$ é $\theta$-convergente a $L(P ; 1)$ e $\left(L\left(P_{n} ; 0\right)\right)_{n \in \mathbb{N}}$ é $\theta$-convergente a $L(P ; 0)$.

Caso tenhamos $\mathbb{K}=\mathbb{R}$ e $k$ par, ainda é necessário supor que $\left(L\left(P_{n} ;-1\right)\right)_{n \in \mathbb{N}}$ seja $\theta$-convergente a $L(P ;-1)$. De fato, basta observar que, nesse caso, se $\alpha<0$, temos $L(P ; \alpha)=(-\alpha)^{\frac{1}{k}} L(P ;-1)$.

Proposição 5.3. Sejam $P_{n} \in \mathcal{P}\left({ }^{r_{n}} E\right)$ e $P \in \mathcal{P}(E)$, e suponhamos que $P_{n} \rightarrow P$ pontualmente. Então:

1. Se $\left(r_{n}\right)_{n \in \mathbb{N}}$ é ilimitada, então $P \equiv 0$

2. Se $P \not \equiv 0$, então $\lim _{n \rightarrow \infty} r_{n}=r_{0}$ e $P \in \mathcal{P}\left({ }^{r o} E\right)$. 
Demonstração. (1) Seja $x \in E$. Sem perda de generalidade, podemos assumir $r_{n} \rightarrow \infty$. Temos:

$$
P(2 x)=\lim _{n \rightarrow \infty} P_{n}(2 x)=\lim _{n \rightarrow \infty}\left[2^{r_{n}} P_{n}(x)\right]=P(x) \lim _{n \rightarrow \infty} 2^{r_{n}} .
$$

Logo, $P(x)=0$ e, como $x$ era arbitrário, segue que $P \equiv 0$.

(2) Assumamos que $\left(r_{n}\right)_{n \in \mathbb{N}}$ é limitada. Se $r_{a}$ e $r_{b}$ são pontos de acumulação de $\left(r_{n}\right)_{n \in \mathbb{N}}$, escolha $x_{0} \in E$ tal que $P\left(x_{0}\right) \neq 0$, e temos:

$$
P\left(2 x_{0}\right)=\lim _{k \rightarrow \infty}\left[2^{r_{n_{k}}} P_{n_{k}}\left(x_{0}\right)\right]=2^{r_{a}} P\left(x_{0}\right) .
$$

Analogamente, $P\left(2 x_{0}\right)=2^{r_{b}} P\left(x_{0}\right)$. Logo, $r_{a}=r_{b}$, e $\left(r_{n}\right)_{n \in \mathbb{N}}$ é convergente. Sejam $x \in E$, $\lambda \in \mathbb{K}$ arbitrários e $r_{0}=\lim _{n \rightarrow \infty} r_{n}$. Então:

$$
P(\lambda x)=\lim _{n \rightarrow \infty} P_{n}(\lambda x)=\lim _{n \rightarrow \infty} \lambda^{r_{n}} P(x)=\lambda^{r_{0}} P(x) .
$$

Como $P \in \mathcal{P}(E)$, concluímos que $P \in \mathcal{P}\left({ }^{r_{0}} E\right)$.

\subsection{Convergência no sentido de Kuratowski}

Essa seção será dedicada a estabelecer relações entre convergência de polinômios e a convergência, no sentido de Kuratowski, de seus conjuntos de nível. O objetivo inicial da seção é a obtenção de um resultado para polinômios análogo ao teorema 3.4.

Proposição 5.4. Sejam $E$ um espaço de Banach sobre $\mathbb{K},\left(P_{n}\right)_{n \in \mathbb{N}}$ uma seqüência tal que $P_{n} \in$ $\mathcal{P}\left({ }^{r_{n}} E\right), \forall n \in \mathbb{N}$ e $P_{0}$ uma função contínua. Suponhamos que, $\forall \alpha \neq 0, P_{n} \stackrel{\mathrm{K}}{\longrightarrow} P_{0}$, e que $r_{n} \rightarrow \infty$. Então, $P_{0} \equiv 0$.

Demonstração. Sejam $\alpha \neq 0$ e $x \in E$ tal que $P_{0}(x)=\alpha$. Pela proposição 2.16, que caracteriza a convergência no sentido de Kuratowski, existe uma seqüência $\left(x_{n}\right)_{n \in \mathbb{N}} \subset E$ com $x_{n} \rightarrow x$ e $P_{n}\left(x_{n}\right)=\alpha, \forall n \in \mathbb{N}$.

Considere $\epsilon_{n}=2^{\frac{1}{r_{n}}}$. Claramente, $\epsilon_{n} \rightarrow 1$, e $\epsilon_{n} x_{n} \rightarrow x$. Mas $P_{n}\left(\epsilon_{n} x_{n}\right)=\epsilon_{n}^{r_{n}} P_{n}\left(x_{n}\right)=2 \alpha$. Logo, $\epsilon_{n} x_{n} \in L\left(P_{n} ; 2 \alpha\right)$ e, pela segunda condição da proposição $2.16, x \in L\left(P_{0} ; 2 \alpha\right)$, donde $P_{0}(x)=2 \alpha$, contradição.

Os dois lemas seguintes tratam sobre convergência uniforme de funções em subconjuntos limitados, e podem ser enunciados em um contexto muito mais geral do que aquele em que estamos trabalhando. A equivalência que esses resultados apresentam será utilizada na próxima proposição. 
Lema 5.5. Seja $X$ um espaço métrico. Sejam $f, f_{n}: X \rightarrow \mathbb{R}$ funções contínuas em $X$ tais que $\left(f_{n}\right)_{n \in \mathbb{N}}$ converge uniformemente a $f$. Se $\left(x_{n}\right)_{n \in \mathbb{N}} \subset X$ é tal que $x_{n} \rightarrow x \in X$, então $f(x)=$ $\lim _{n \rightarrow \infty} f_{n}\left(x_{n}\right)$.

Demonstração. Pela convergência uniforme, dado $\epsilon>0$, existe $n_{0} \in \mathbb{N}$ tal que $\left|f_{n}(y)-f(y)\right|<\frac{\epsilon}{2}$, $\forall y \in X, \forall n>n_{0}$. Em particular, se tomarmos $y=x_{n}$, temos:

$$
\forall n>n_{0},\left|f_{n}\left(x_{n}\right)-f\left(x_{n}\right)\right|<\frac{\epsilon}{2}
$$

Como $x_{n} \rightarrow x$, existe $n_{1} \in \mathbb{N}$ tal que:

$$
\forall n>n_{1},\left|f\left(x_{n}\right)-f(x)\right|<\frac{\epsilon}{2}
$$

Tomando $n_{2}=\max \left\{n_{0}, n_{1}\right\}$, temos, se $n>n_{2}$ :

$$
\left|f_{n}\left(x_{n}\right)-f(x)\right| \leq\left|f_{n}\left(x_{n}\right)-f\left(x_{n}\right)\right|+\left|f\left(x_{n}\right)-f(x)\right|<\epsilon
$$

e segue o resultado.

Lema 5.6. Seja $X$ um espaço métrico, e sejam $f, f_{n}: X \rightarrow \mathbb{R}$ funções contímuas em $X$ tais que:

$$
\left(x_{n}\right)_{n \in \mathbb{N}} \subset X, x_{n} \rightarrow x \text { implica } f(x)=\lim _{n \rightarrow \infty} f_{n}\left(x_{n}\right) .
$$

Então, $\left(f_{n}\right)_{n \in \mathbb{N}}$ converge uniformemente a $f$ em cada subconjunto compacto de $X$.

Demonstração. Vamos proceder por absurdo. Suponhamos que existe $K \subset X$ compacto no qual a seqüência $\left(f_{n}\right)_{n \in \mathbb{N}}$ não converge uniformemente a $f$. Dessa forma, existe $\epsilon>0$ tal que, para cada $n \in \mathbb{N}$, existe $x_{n} \in K$ tal que $\left|f_{n}\left(x_{n}\right)-f\left(x_{n}\right)\right| \geq \epsilon$.

Como $K$ é compacto, a seqüência $\left(x_{n}\right)_{n \in \mathbb{N}}$ admite uma subseqüência $\left(x_{n_{k}}\right)_{k \in \mathbb{N}}$ que converge a $x \in K$. Por hipótese, temos:

$$
\lim _{k \rightarrow \infty} f\left(x_{n_{k}}\right)=f(x)
$$

Então, existe $n_{k_{0}} \in \mathbb{N}$ tal que $\left|f_{n_{k}}\left(x_{n_{k}}\right)-f(x)\right|<\frac{\epsilon}{2}, \forall n_{k}>n_{k_{0}}$. Assim:

$$
\left|f(x)-f\left(x_{n_{k}}\right)\right| \geq\left|f(x)-f_{n_{k}}\left(x_{n_{k}}\right)\right|-\left|f_{n_{k}}\left(x_{n_{k}}\right)-f\left(x_{n_{k}}\right)\right|>\epsilon-\frac{\epsilon}{2}=\frac{\epsilon}{2}, \forall n_{k}>n_{k_{0}},
$$

absurdo, pois $f$ é contínua.

A proposição seguinte mostra que, sob certas hipóteses, a convergência de funções no sentido de Kuratowski implica a convergência uniforme das funções em subconjuntos compactos (note-se a equivalência da conclusão da proposição com esse fato, dada pelos lemas 5.5 e 5.6). 
Proposição 5.7. Sejam $E$ um espaço de Banach real, $\left(f_{n}\right)_{n \in \mathbb{N}}$ uma seqüência de funções contínuas em $E$ e $f$ uma função contínua em $E$ tais que $f_{n} \stackrel{\mathrm{K}}{\longrightarrow} f$. Então, $f(x)=\lim _{n \rightarrow \infty} f_{n}\left(x_{n}\right)$, para toda seqüência $\left(x_{n}\right)_{n \in \mathbb{N}} \subset E$ tal que $x_{n} \rightarrow x$.

Demonstração. Seja $\alpha=f(x)$. Se a seqüência $\left(f_{n}\left(x_{n}\right)\right)_{n \in \mathbb{N}}$ não converge a $\alpha$, podemos assumir, passando a uma subseqüência se necessário, que existe $\epsilon>0$ tal que $f_{n}\left(x_{n}\right)>\alpha+\epsilon, \forall n \in \mathbb{N}$.

Usando a condição $i$ ) da proposição 2.16 , podemos escolher uma seqüiência $\left(z_{n}\right)_{n \in \mathbb{N}} \subset E$ convergente a $x$ tal que $f_{n}\left(z_{n}\right)=\alpha, \forall n \in \mathbb{N}$.

Considere, agora, a função $\Lambda:[0,1] \longrightarrow E$ definida por $\Lambda(\lambda)=\lambda x_{n}+(1-\lambda) z_{n}$. É claro que $\Lambda$ é contímua. Seja $g_{n}=f_{n} \circ \Lambda$.

Então, $g_{n}:[0,1] \longrightarrow \mathbb{R}$ é contínua, $g_{n}(0)=f_{n}\left(z_{n}\right)=\alpha$ e $g_{n}(1)=f_{n}\left(x_{n}\right)>\alpha+\epsilon$. Logo, pelo Teorema do Valor Intermediário, existe $\lambda_{n} \in[0,1]$ tal que $g_{n}\left(\lambda_{n}\right)=\alpha+\frac{\epsilon}{2}$.

Definamos $y_{n}=\Lambda\left(\lambda_{n}\right)$. Então, $y_{n} \rightarrow x$ e $f_{n}\left(y_{n}\right)=\alpha+\frac{\epsilon}{2}, \forall n \in \mathbb{N}$. Utilizando a condição $\left.i i\right)$ da proposição 2.16 , obtemos $f(x)=\alpha+\frac{\epsilon}{2}$, absurdo.

O caso complexo é mais complicado, como mostra o seguinte exemplo:

Exemplo 5.8. Seja $w_{n}=e^{\frac{2 \pi i}{n}}$. Definamos $f_{n}(w)=\phi_{n}(|w|)$, onde:

$$
\phi_{n}(r)=\left\{\begin{array}{cl}
(1-n r) w_{n} & , 0 \leq r \leq \frac{1}{n} \\
0 & , r>\frac{1}{n}
\end{array}\right.
$$

Note que $f_{n} \stackrel{K}{\longrightarrow} 0$, pois, se $\alpha \neq 0$, o conjunto $L\left(f_{n} ; \alpha\right)$ é não-vazio para, no máximo, um termo n. Além disso, $\mathbb{C}=\mathrm{K}-\lim _{n \in \mathbb{N}} L\left(f_{n} ; 0\right)$.

De fato, tome $\alpha \neq 0$. Se $w \in \mathbb{C}$ é tal que $f_{n}(w)=\alpha$, então $(1-n|w|) w_{n}=\alpha$, donde $(1-n|w|) e^{\frac{2 \pi i}{n}}=\alpha$. Logo,

$$
(1-n|w|)\left(\cos \left(\frac{2 \pi}{n}\right)+i \operatorname{sen}\left(\frac{2 \pi}{n}\right)\right)=|\alpha|(\cos \theta+i \operatorname{sen} \theta)
$$

Como $|w|<\frac{1}{n}$, temos $1-n|w|>0$ e, portanto, $1-n|w|=|\alpha|$, e $n$ está unicamente determinado, caso exista.

Além disso, $L\left(f_{n} ; 0\right)=\left\{w \in \mathbb{C}: f_{n}(w)=0\right\} \supseteq\left\{w \in \mathbb{C}:|w| \geq \frac{1}{n}\right\}$. Se $n \rightarrow \infty$, é fácil ver que $\mathbb{C}=\mathrm{K}-\lim _{n \in \mathbb{N}} L\left(f_{n} ; 0\right)$.

No entanto, $\left(f_{n}(0)\right)_{n \in \mathbb{N}}$ converge a 1 . De fato:

$$
f_{n}(0)=\phi_{n}(0)=(1-n .0) w_{n}=e^{\frac{2 \pi i}{n}} .
$$

Fazendo $n \rightarrow \infty$, tem-se $f_{n}(0) \rightarrow 1$. 
É possível obter um resultado semelhante no caso complexo, de acordo com [8]. No entanto, esse resultado utiliza conceitos de holomorfia em espaços de Banach, assunto que foge ao escopo desse trabalho. Uma versão mais simples desse resultado, e que basta ao nosso contexto, será enunciada na proposição a seguir:

Proposição 5.9. Sejam $E$ um espaço de Banach complexo, $\left(P_{n}\right)_{n \in \mathbb{N}}$ uma seqüência de polinômios em $\mathcal{P}(E)$ e $P \in \mathcal{P}(E)$. Suponhamos que $\forall B \subset E$ limitado exista uma constante $M>0$ tal que:

$$
\left\|d P_{n}(y)\right\| \leq M, \forall y \in B, \forall n \in \mathbb{N}
$$

Se $P_{n} \stackrel{K}{\longrightarrow} P$, então $P(x)=\lim _{n \rightarrow \infty} P_{n}\left(x_{n}\right)$, para toda seqüência $\left(x_{n}\right)_{n \in \mathbb{N}} \subset E$ tal que $x_{n} \rightarrow x$.

Demonstração. Sejam $\left(x_{n}\right)_{n \in \mathbb{N}} \subset E, x \in E$ tais que $x_{n} \rightarrow x$ e $\alpha=P(x)$. Pela proposição 2.16, existe uma sequiência $\left(z_{n}\right)_{n \in \mathbb{N}}$ tal que $P_{n}\left(z_{n}\right)=\alpha, \forall n \in \mathbb{N}$, e $z_{n} \rightarrow x$. Temos, pelo teorema 4.25:

$$
\left|P_{n}\left(x_{n}\right)-P_{n}\left(z_{n}\right)\right| \leq\left\|d P_{n}\left(y_{n}\right)\right\|\left\|x_{n}-z_{n}\right\| \leq M\left\|x_{n}-z_{n}\right\|
$$

onde $y_{n} \in\left[x_{n}, z_{n}\right]$. Como $x_{n} \rightarrow x$ e $z_{n} \rightarrow x$, segue que $y_{n} \rightarrow x$, donde podemos assumir que $y_{n} \in B(0 ;\|x\|+1)$, para $n$ suficientemente grande. Fazendo $n \rightarrow \infty$, temos que $z_{n} \rightarrow x$ e, portanto, $P_{n}\left(x_{n}\right) \rightarrow \alpha$.

A próxima proposiçâo nos diz o que ocorre com os polinômios quando assumimos a convergência, no sentido de Kuratowski, de seus conjuntos de nível.

Proposição 5.10. Sejam $E$ um espaço de Banach, $\left(P_{n}\right)_{n \in \mathbb{N}}$ uma seqüência tal que $P_{n} \in \mathcal{P}\left({ }^{r_{n}} E\right)$, $\forall n \in \mathbb{N}$ e $P \in \mathcal{P}\left({ }^{r_{0}} E\right)$. Suponha que $\forall \alpha \neq 0, L(P ; \alpha)=\mathrm{K}-\lim _{n \in \mathbb{N}} L\left(P_{n} ; \alpha\right)$. Então:

1. $P_{n}\left(x_{n}\right) \rightarrow P(x)$ sempre que $\left(x_{n}\right)_{n \in \mathbb{N}} \subset E$ é tal que $x_{n} \rightarrow x$

2. Se $P \not \equiv 0$, então $\left(\left\|P_{n}\right\|\right)_{n \in \mathbb{N}}$ é limitada.

Demonstração. Vamos, inicialmente, demonstrar o item (2). Se $\left(\left\|P_{n}\right\|\right)_{n \in \mathbb{N}}$ não é limitada, podemos assumir, sem perda de generalidade, que existe uma seqüência $\left(x_{n}\right)_{n \in \mathbb{N}} \subset E$, com $\left\|x_{n}\right\|=1$, tal que $P_{n}\left(x_{n}\right)=d_{n}$, com $d_{n} \rightarrow \infty$. Cabe notar que, no caso real, pode ser necessário substituir $P_{n}$ por $-P_{n}$ e $P$ por $-P$ para fazermos o que segue.

Seja $z_{n}=d_{n}^{\frac{-1}{r_{n}}} x_{n}$. É fácil ver que $z_{n} \in L\left(P_{n} ; 1\right)$. Como $\left(r_{n}\right)_{n \in \mathbb{N}}$ é limitada, pela proposição 5.4 (se $r_{n} \rightarrow \infty$, teríamos $P \equiv 0$, absurdo), segue que $z_{n} \rightarrow 0$. Pela condição ii) da proposição 2.16, concluímos que $P(0)=1$, o que não é possível, pois $P$ é homogêneo.

Vamos, agora, à demonstração de (1). Denotemos $P(x)=\beta$ e $P_{n}\left(x_{n}\right)=\beta_{n}$. Se $\beta=0$, devemos mostrar que $\beta_{n} \rightarrow 0$. Se isso não é verdade, podemos assumir que $\left|\beta_{n}\right|>\epsilon$, para algum $\epsilon>0$ 
fixado. Seja $\alpha_{n}=\beta_{n}^{\frac{-1}{r_{n}}}$. A seqüência $\left(\alpha_{n}\right)_{n \in \mathbb{N}}$ é limitada. Assim, passando a uma subseqüência se necessário, podemos assumir que $\alpha_{n} \rightarrow \alpha \in \mathbb{K}$.

Definamos, agora, $z_{n}=\alpha_{n} x_{n}$. Então:

$$
P_{n}\left(z_{n}\right)=P_{n}\left(\alpha_{n} x_{n}\right)=\left(\beta_{n}^{\frac{-1}{r_{n}}}\right)^{r_{n}} P_{n}\left(x_{n}\right)=\beta_{n}^{-1} \beta_{n}=1
$$

donde $z_{n} \in L\left(P_{n} ; 1\right)$. Como $z_{n} \rightarrow \alpha x$, temos $P(\alpha x)=1$, uma vez que estamos assumindo $L(P ; 1)=\mathrm{K}-\lim _{n \in \mathbb{N}} L\left(P_{n} ; 1\right)$. Mas $P(x)=\beta=0$, donde $P(\alpha x)=0$, absurdo. Note-se que, para o caso real, algumas pequenas alterações nas definições de $\alpha_{n}$ e de $z_{n}$ devem ser efetuadas.

Se $\beta \neq 0$, a proposição 5.7 fornece o resultado para o caso real. Pela proposição 5.9, para obtermos o resultado no caso complexo basta mostrarmos que $\left(\left\|d P_{n}(y)\right\|\right)_{n \in \mathbb{N}}$ é uniformemente limitada em conjuntos limitados. Se $A_{n}$ é a forma $r_{n}$-linear simétrica associada a $P_{n}$, temos, pelo exemplo 4.23 e, em seguida, pela proposição 4.19 :

$$
\left\|d P_{n}(y)\right\| \leq r_{n}\|y\|^{r_{n}-1}\left\|A_{n}\right\| \leq r_{n}\|y\|^{r_{n}-1} \frac{r_{n}^{r_{n}}}{r_{n} !}\left\|P_{n}\right\| .
$$

Mas então $\left(\left\|d P_{n}(y)\right\|\right)_{n \in \mathbb{N}}$ é uniformemente limitada em conjuntos limitados, pois $\left(r_{n}\right)_{n \in \mathbb{N}}$ e $\left(\left\|P_{n}\right\|\right)_{n \in \mathbb{N}}$ são limitadas.

Essa proposição, juntamente com a proposição 5.3, fornece as seguintes alternativas: ou $P \equiv 0$ ou $r_{n}=r_{0}$, para $n$ suficientemente grande. Dessa forma, podemos restringir o estudo a dois casos, não mutuamente exclusivos:

1. $r_{n}=k, \forall n \in \mathbb{N}$;

2. $P \equiv 0$.

O caso $k=0$ será excluído. O exemplo seguinte mostra que, se $P \equiv 0$, não se pode afirmar que $\left(\left\|P_{n}\right\|\right)_{n \in \mathbb{N}}$ é limitada. Para construir esse exemplo, é necessário conhecer o teorema de JosefsonNissenzweig. Esse teorema será enunciado a seguir, mas sua demonstração será omitida, por fugir ao escopo desse trabalho. Tal demonstração pode ser encontada à página 219 de [7]:

Teorema 5.11. (Josefson-Nissenzweig) Se E é um espaço de Banach de dimensão infinita, existe uma seqüência fraca-ネ nula de vetores unitários em $E^{\prime}$.

Por seqüência fraca- $\star$ nula em $E^{\prime}$, entendemos uma seqüência que converge a zero em cada ponto do espaço $E$ em questão, isto é, $\left(f_{n}\right)_{n \in \mathbb{N}} \subset E^{\prime}$ é fraca- $\star$ nula se, para cada $x \in E, f_{n}(x) \rightarrow 0$. 
Exemplo 5.12. Vamos mostrar que o fato de que $P_{k} \stackrel{\mathrm{K}}{\longrightarrow} P$ não nos permite afirmar que $\left(\left\|P_{n}\right\|\right)_{n \in \mathbb{N}}$ é limitada. Seja $E$ um espaço de Banach com $\operatorname{dim}_{\mathbb{K}} E=+\infty$. Pelo teorema 5.11 , existe uma seqüência fraca- $\star$ nula $\left(x_{n}^{\star}\right)_{n \in \mathbb{N}}$ na esfera unitária de $E^{\prime}$. Definamos:

$$
P_{k}(x)=k\left(x_{k}^{\star}(x)\right)^{k} \quad \text { e } \quad P \equiv 0 .
$$

É claro que $P_{k} \in \mathcal{P}\left({ }^{k} E\right), \forall k \in \mathbb{N}$, e que $\left\|P_{k}\right\|=k, \forall k \in \mathbb{N}$. Além disso, $L\left(P_{k} ; 0\right)=\{x \in E$ : $\left.k\left(x_{k}^{\star}(x)\right)^{k}=0\right\}=\operatorname{ker} x_{k}^{\star}$.

Como $\left\|x_{k}^{\star}\right\|=1$, temos, pelo lema 3.9 , que $\left|x_{k}^{\star}(x)\right|=d\left(x\right.$, ker $\left.x_{k}^{\star}\right)$. Então, existe $z_{k} \in L\left(P_{k} ; 0\right)$ tal que $\left(z_{k}\right)_{k \in \mathbb{N}}$ converge a $x$, pois $\lim _{k \rightarrow \infty} d\left(x, \operatorname{ker} x_{k}^{\star}\right)=0$, donde conclui-se que $L(P ; 0)=\mathrm{K}-\lim _{n \in \mathbb{N}} L\left(P_{k} ; 0\right)$, já que a condição $i i)$ da proposição 2.16 é trivialmente satisfeita, pois $E=L(P ; 0)$.

Escrevamos:

$$
L(P ; 1)=\emptyset \quad \text { e } \quad L\left(P_{k} ; 1\right)=\left\{x \in E: x_{k}^{\star}(x)=\left(\frac{1}{k}\right)^{\frac{1}{k}}\right\}
$$

Para mostrar que $P_{k} \stackrel{\mathrm{K}}{\longrightarrow} P$, precisamos mostrar que $\left(x_{k}\right)_{k \in \mathbb{N}}$ não possui subseqüências convergentes se cada $x_{k} \in L\left(P_{k} ; 1\right)$. Como $\left(x_{k}^{\star}\right)_{k \in \mathbb{N}}$ é uma seqüência fraca- $\star$ nula limitada, temos que, se $x=\lim _{n \rightarrow \infty} x_{k_{n}}$, então $\lim _{n \rightarrow \infty} x_{k_{n}}^{\star}\left(x_{k_{n}}\right)=0$, o que não é verdade, pois $x_{k_{n}}^{\star}\left(x_{k_{n}}\right)=\left(\frac{1}{k_{n}}\right)^{\frac{1}{k_{n}}}$. Note-se que, para evitar problemas com o caso real, devemos considerar $k$ ímpar.

Cabe ressaltar que, se $E$ é um espaço de dimensão finita, a compacidade dos subconjuntos fechados e limitados nos garante a convergência da norma de $P_{k}$ a 0 , se $P_{k} \stackrel{\mathrm{K}}{\longrightarrow} 0$.

Para poder dizer mais sobre o comportamento da norma dos elementos de seqüências de polinômios convergentes no sentido de Kuratowski, precisamos da seguinte:

Proposição 5.13. Sejam $E$ um espaço de Banach, $\left(P_{n}\right)_{n \in \mathbb{N}}$ uma seqüência tal que $P_{n} \in \mathcal{P}\left({ }^{r_{n}} E\right)$, $\forall n \in \mathbb{N}$ e $P$ um polinômio contínuo em $E$. Se, $\forall \alpha \neq 0, L(P ; \alpha)=\mathrm{K}-\lim _{n \in \mathbb{N}} L\left(P_{n} ; \alpha\right)$ e $\left(x_{n}\right)_{n \in \mathbb{N}} \subset E$ é limitada, então $\left(\mid P_{n}\left(x_{n}\right)^{\frac{1}{r_{n}}}\right)_{n \in \mathbb{N}}$ também é limitada.

Demonstração. Inicialmente, vamos supor que $E$ é um espaço de Banach complexo. Sejam $\alpha_{n}=P_{n}\left(x_{n}\right), \forall n \in \mathbb{N}$, e seja $\alpha \neq 0$. Assumamos que $\left(\left|\alpha_{n}\right|^{\frac{1}{r_{n}}}\right)_{n \in \mathbb{N}}$ não é limitada, e definamos $\beta_{n}=\alpha \alpha_{n}^{-1}$. Temos:

$$
P_{n}\left(\beta_{n}^{\frac{1}{r_{n}}} x_{n}\right)=\left(\beta_{n}^{\frac{1}{r_{n}}}\right)^{r_{n}} P_{n}\left(x_{n}\right)=\beta_{n} \alpha_{n}=\alpha \Rightarrow \beta_{n}^{\frac{1}{r_{n}}} x_{n} \in L\left(P_{n} ; \alpha\right)
$$

Como $\left(\left|\alpha_{n}\right|^{\frac{1}{r_{n}}}\right)_{n \in \mathbb{N}}$ não é limitada, uma subseqüência de $\left(\beta_{n}^{\frac{1}{r_{n}}}\right)_{n \in \mathbb{N}}$, e, conseqüentemente, uma subsequiência de $\left(\beta_{n}^{\frac{1}{r_{n}}} x_{n}\right)_{n \in \mathbb{N}}$, converge a zero. Mas então, pela caracterização da convergência no sentido de Kuratowski dada pela proposição $2.16,0 \in L(P ; \alpha)$. 
Observe, agora, que $P_{n}$ converge a $P$ pontualmente (basta notar que estamos no caso $P(x) \neq 0$ da proposição 5.10, que não exige a homogeneidade de $P$, pois só usa a proposição 5.9). Assim, pela proposição $5.3, P$ é um polinômio homogêneo, o que contradiz o fato de que $0 \in L(P ; \alpha)$.

Note-se que algumas pequenas alterações devem ser efetuadas para provarmos o caso em que $E$ é um espaço de Banach real, em função da necessidade de extrairmos raízes de elementos do corpo.

Os corolários seguintes são imediatos:

Corolário 5.14. Sejam $E$ um espaço de Banach, $\left(P_{n}\right)_{n \in \mathbb{N}}$ uma seqüência tal que $P_{n} \in \mathcal{P}\left({ }^{r_{n}} E\right)$, $\forall n \in \mathbb{N}$ e $P$ um polinômio contínuo em $E$. Se, $\forall \alpha \neq 0, L(P ; \alpha)=\mathrm{K}-\lim _{n \in \mathbb{N}} L\left(P_{n} ; \alpha\right)$, então $\left(\left\|P_{n}\right\|^{\frac{1}{r_{n}}}\right)_{n \in \mathbb{N}}$ é limitada.

Demonstração. Suponhamos, por absurdo, que $\left(\left\|P_{n}\right\|^{\frac{1}{r_{n}}}\right)_{n \in \mathbb{N}}$ não é limitada. Então, para cada $n \in \mathbb{N}$, existe $x_{n} \in E$, com $\left\|x_{n}\right\|=1$, tal que $\mid P_{n}\left(x_{n}\right)^{\frac{1}{r_{n}}}=d_{n}$ e $d_{n} \rightarrow \infty$, o que contradiz a proposição anterior.

Corolário 5.15. Seja $E$ um espaço de Banach. Se $\left(P_{n}\right)_{n \in \mathbb{N}} \subset P\left({ }^{k} E\right), P \in \mathcal{P}\left({ }^{k} E\right)$ e $L(P ; \alpha)=$ $\mathrm{K}-\lim _{n \in \mathbb{N}} L\left(P_{n} ; \alpha\right), \forall \alpha \neq 0$, então $\left(\left\|P_{n}\right\|\right)_{n \in \mathbb{N}}$ é limitada.

Demonstração. Pelo corolário 5.14, temos que $\left(\left\|P_{n}\right\|^{\frac{1}{k}}\right)_{n \in \mathbb{N}}$ é limitada. Como $k$ é fixo, $\left(\left\|P_{n}\right\|\right)_{n \in \mathbb{N}}$ também deve ser limitada.

O exemplo 5.12 mostrou que o fato de termos $P_{n} \stackrel{\mathrm{K}}{\longrightarrow} 0$ não garante que $\lim _{n \rightarrow \infty}\left\|P_{n}\right\|^{\frac{1}{r_{n}}}=0$. No entanto, temos a seguinte recíproca:

Proposição 5.16. Sejam $E$ um espaço de Banach, $\left(P_{n}\right)_{n \in \mathbb{N}}$ uma seqüência tal que $P_{n} \in \mathcal{P}\left({ }^{r_{n}} E\right)$, $\forall n \in \mathbb{N}$ e $P \in \mathcal{P}(E)$. Se $L(P ; \alpha)=\mathrm{K}-\lim _{n \in \mathbb{N}} L\left(P_{n} ; \alpha\right), \forall \alpha \neq 0$ e $\lim _{n \rightarrow \infty}\left\|P_{n}\right\|^{\frac{1}{r_{n}}}=0$, então $P \equiv 0$.

Demonstração. Assumamos que $\left(r_{n}\right)_{n \in \mathbb{N}}$ é limitada (caso contrário, a proposição 5.4 já garante o resultado), e seja $x \in E$ com $P(x)=\alpha \neq 0$. Por hipótese, existe uma seqüência $\left(x_{n}\right)_{n \in \mathbb{N}} \subset E$ convergente a $x$, e então limitada em norma por uma constante $M>0$, tal que $x_{n} \in L\left(P_{n} ; \alpha\right), \forall n \in$ $\mathbb{N}$. $\log o, P_{n}\left(x_{n}\right)=P(x), \forall n \in \mathbb{N}$. Então:

$$
|P(x)|=\left|P_{n}\left(x_{n}\right)\right| \leq\left\|P_{n}\right\|\left\|x_{n}\right\|^{r_{n}} \leq\left\|P_{n}\right\| M^{r_{n}}=\left(\left\|P_{n}\right\|^{\frac{1}{r_{n}}} M\right)^{r_{n}} .
$$

Como $\left(r_{n}\right)_{n \in \mathbb{N}}$ é limitada, segue que $\left(\left\|P_{n}\right\|^{\frac{1}{r_{n}}} M\right)^{r_{n}} \rightarrow 0$.

A proposição seguinte apresenta uma recíproca da proposição 5.10 : 
Proposição 5.17. Sejam $E$ um espaço de Banach, $\left(P_{n}\right)_{n \in \mathbb{N}}$ uma seqüência tal que $P_{n} \in \mathcal{P}\left({ }^{r_{n}} E\right)$, $\forall n \in \mathbb{N}$ e $P \in \mathcal{P}\left({ }^{r_{0}} E\right)$. Se, para toda seqüência $\left(x_{n}\right)_{n \in \mathbb{N}}$ em $E$ convergente a $x \in E$, se tem $P_{n}\left(x_{n}\right) \rightarrow P(x)$, então $L(P ; \alpha)=\mathrm{K}-\lim _{n \in \mathbb{N}} L\left(P_{n} ; \alpha\right), \forall \alpha \neq 0$.

Demonstração. De acordo com a observação 5.2, é suficiente mostrarmos o caso $\alpha=1$ (o caso $\alpha=-1$ é análogo). Se $P \not \equiv 0$ e $x \in L(P ; 1)$, a seqüência $\left(P_{n}(x)\right)_{n \in \mathbb{N}}$ converge a 1 . Podemos, então, assumir que $P_{n}(x) \neq 0, \forall n \in \mathbb{N}$. Se definirmos

$$
x_{n}=P_{n}(x)^{\frac{-1}{r_{n}}} x
$$

temos $x_{n} \rightarrow x$ e $x_{n} \in L\left(P_{n} ; 1\right), \forall n \in \mathbb{N}$, o que demonstra o item $(i)$ da proposição 2.16 . E, se $\left(x_{n_{k}}\right)_{k \in \mathbb{N}}$ converge a $x$ e $P_{n_{k}}\left(x_{n_{k}}\right)=1, \forall k \in \mathbb{N}$, a hipótese nos garante que $P(x)=1$, donde $L(P ; 1)=\mathrm{K}-\lim _{n \in \mathbb{N}} L\left(P_{n} ; 1\right)$, o que demosntra o item $(i i)$ de 2.16 .

Suponhamos, agora, que $P \equiv 0$. Devemos mostrar que $\emptyset=\mathrm{K}-\lim _{n \in \mathbb{N}} L\left(P_{n} ; 1\right)$. Se $x_{n_{k}} \in$ $L\left(P_{n_{k}} ; 1\right), \forall k \in \mathbb{N}$, então $\left(x_{n_{k}}\right)_{k \in \mathbb{N}}$ não pode ser convergente, pois, se fosse, a imagem de seu limite por $P$ seria 1 (por hipótese), o que contradiz o fato de termos $P \equiv 0$.

Observe que as proposições 5.10 e 5.17, em conjunto, nos fornecem um resultado análogo, no contexto de polinômios, àquele obtido no teorema 3.5. Basta notar que a convergência pontual de polinômios corresponde à convergência fraca-^ de funcionais lineares contínuos.

Agregando os resultados anteriores, obtemos o teorema pelo qual buscávamos ao longo desse seção:

Teorema 5.18. Seja $E$ um espaço de Banach sobre $\mathbb{K}$, e sejam $\left(P_{n}\right)_{n \in \mathbb{N}}$ uma seqüência tal que $P_{n} \in \mathcal{P}\left({ }^{r_{n}} E\right), \forall n \in \mathbb{N}$ e $P \in \mathcal{P}\left({ }^{r_{0}} E\right)$. São equivalentes:

1. $L(P ; \alpha)=\mathrm{K}-\lim _{n \in \mathbb{N}} L\left(P_{n} ; \alpha\right), \forall \alpha \neq 0$

2. Para toda seqüência $\left(x_{n}\right)_{n \in \mathbb{N}}$ convergente a $x \in E, \lim _{n \rightarrow \infty} P_{n}\left(x_{n}\right)=P(x)$.

3. A seqüência $\left(P_{n}\right)_{n \in \mathbb{N}}$ converge uniformemente a $P$ em subconjuntos compactos de $E$.

Além disso, se supusermos que $P_{n}, P$ são polinômios não-nulos, as condições acima são equivalentes a:

4. A seqüência $\left(P_{n}\right)_{n \in \mathbb{N}}$ converge pontualmente a $P$ e $\left(\left\|P_{n}\right\|\right)_{n \in \mathbb{N}}$ é limitada.

Esse teorema não afirma nada sobre a convergência de $L\left(P_{n} ; 0\right)$. O comportamento distinto no caso $\alpha=0$ está relacionado ao fato de 0 ser o único valor crítico $(\exists x \in L(P ; 0)$ tal que $d P(x)=0)$ 
de um polinômio homogêneo. Dessa forma, é possível haver mudanças na topologia de $L\left(P_{n} ; \alpha\right)$ em vizinhanças do ponto $\alpha=0$.

O exemplo seguinte mostra que, no caso $P \equiv 0$, podemos não ter a convergência de $L\left(P_{n} ; 0\right)$ a $L(P ; 0)$, mesmo assumindo hipóteses fortes.

Exemplo 5.19. Sejam $E=\mathbb{K}^{2}$ e $P_{n}(x, y)=\frac{x^{2}}{n}$. Observe que, $\forall n \in \mathbb{N}, P_{n}$ é um polinômio 2-homogêneo, e que $\lim _{n \rightarrow \infty}\left\|P_{n}\right\|=0$. No entanto, $\mathbb{K}^{2}=L(0 ; 0) \neq K-\lim _{n \in \mathbb{N}} L\left(P_{n} ; 0\right)$. De fato, $\left(x_{0}, y_{0}\right)=(1,0) \in L(0 ; 0)$. Mas, se $(x, y) \in L\left(P_{n} ; 0\right)$, então $x=0$, e não pode existir uma seqüência $\left(x_{n}, y_{n}\right)_{n \in \mathbb{N}}, \operatorname{com}\left(x_{n}, y_{n}\right) \rightarrow\left(x_{0}, y_{0}\right)$, tal que $\left(x_{n}, y_{n}\right) \in L\left(P_{n} ; 0\right), \forall n \in \mathbb{N}$.

A seguir, vamos apresentar uma recíproca da proposição 5.9. A demonstração desse resultado utiliza um corolário do teorema de Hurwitz, cujo enunciado está a seguir. Por fugir ao escopo desse trabalho, sua demonstração será omitida, mas pode ser encontrada em [6], à página 152. Por $\mathcal{H}(G)$, denotamos o conjunto das funções holomorfas em um subconjunto aberto e conexo $G$ do plano.

Teorema 5.20. (Hurwitz) Seja $G \subseteq \mathbb{C}$ um subconjunto aberto e conexo do plano. Suponhamos que a seqüência $\left(f_{n}\right)_{n \in \mathbb{N}} \subset \mathcal{H}(G)$ convirja uniformemente a $f \in \mathcal{H}(G)$. Se $f \not \equiv 0, B[a, r] \subset G$ e $f(z) \neq 0, \forall z \in G$ tal que $|z-a|=r$, existe $n_{0} \in \mathbb{N}$ tal que, se $n>n_{0}, f$ e $f_{n}$ possuem o mesmo número de zeros em $B(a, r)$.

O seguinte corolário é imediato:

Corolário 5.21. Seja $G \subseteq \mathbb{C}$ um subconjunto aberto e conexo do plano. Suponhamos que a seqüência $\left(f_{n}\right)_{n \in \mathbb{N}} \subset \mathcal{H}(G)$ convirja uniformemente a $f \in \mathcal{H}(G)$ e que cada $f_{n}$ não possua zeros em $G$. Então, $f \equiv 0$ ou $f$ nunca se anula em $G$.

Proposição 5.22. Sejam $E$ um espaço de Banach complexo, $\left(P_{n}\right)_{n \in \mathbb{N}} \subset \mathcal{P}(E) \backslash\{0\}$ e $P \in \mathcal{P}(E) \backslash$ $\{0\}$. Suponhamos que, para toda seqüência $\left(x_{n}\right)_{n \in \mathbb{N}}$ convergente a $x \in E, \lim _{n \rightarrow \infty} P_{n}\left(x_{n}\right)=P(x)$. Então, $P_{n} \stackrel{\mathrm{K}}{\longrightarrow} P$.

Demonstração. Para mostrar essa convergência, vamos utilizar a caracterização da convergência no sentido de Kuratowski fornecida pela proposição 2.16. Em função da hipótese que assumimos, o item ii) dessa proposição é trivialmente satisfeito. Vamos, então, mostrar o item i).

Seja $x \in L(P ; \alpha)$. Suponhamos, por absurdo, que não existe uma seqüência $\left(x_{n}\right)_{n \in \mathbb{N}} \subset E$ convergente a $x$ tal que $P_{n}\left(x_{n}\right)=\alpha, \forall n \in \mathbb{N}$. Podemos, então, assumir que existe $\epsilon>0$ tal que $L\left(P_{n} ; \alpha\right) \cap B(x, \epsilon)=\emptyset, \forall n \in \mathbb{N}$.

Considere os polinômios $Q_{n}=P_{n}-\alpha$ e $Q=P-\alpha$. Então, $L\left(Q_{n} ; 0\right) \cap B(x, \epsilon)=\emptyset, \forall n \in \mathbb{N}$. Considere o subconjunto unidimensional $R_{z}=\{x+\lambda z: \lambda \in \mathbb{C}\}$, e tome $z_{0} \in E$ de forma que $Q$ não 
seja identicamente nula em $R_{z_{0}}$ (observe que esse subconjunto existe, pois, se $Q$ fosse identicamente nula em cada subconjunto da forma $R_{z}$, teríamos $Q$ identicamente nula em uma bola, o que nos daria $Q \equiv 0$, absurdo).

Seja $\Lambda: \mathbb{C} \longrightarrow R_{z_{0}}$ dada por $\Lambda(\lambda)=x+\lambda z_{0} \in R$, e sejam $q_{n}=\left(Q_{n}\right)_{\mid R_{z_{0}}}$ e $q=Q_{\mid R_{z_{0}}}$. Definamos $\Omega=B(x, \epsilon) \cap R_{z_{0}}$. Observe que o conjunto $\Lambda^{-1}(\Omega)$ é aberto e conexo em $\mathbb{C}$, pelas definições de $\Lambda$ e de $\Omega$.

Se escrevermos $\phi=q \circ \Lambda$ e $\phi_{n}=q_{n} \circ \Lambda$, temos que:

- $\left(\phi_{n}\right)_{n \in \mathbb{N}}$ converge uniformemente a $\phi$ em $\Lambda^{-1}(\Omega)$, pois, por hipótese, $\left(Q_{n}\right)_{n \in \mathbb{N}}$ converge uniformemente a $Q$ em subconjuntos compactos (e, em particular, em $\bar{\Omega}$ );

- Por definição, $\phi_{n}$ não se anula em $\Lambda^{-1}(\Omega)$.

Assim, pelo corolário 5.21, temos as seguintes alternativas: ou $\phi$ é identicamente nulo, ou $\phi$ não possui zeros en $\Lambda^{-1}(\Omega)$. Nenhuma delas é verdadeira, pela definição de $R_{z_{0}}$ e pelo fato de que $q(x)=Q(x)=P(x)-\alpha=0$.

Corolário 5.23. Se $E$ é um espaço de Banach complexo, $\left(P_{n}\right)_{n \in \mathbb{N}} \subset \mathcal{P}\left({ }^{k} E\right) \backslash\{0\}, P \in \mathcal{P}\left({ }^{k} E\right) \backslash\{0\}$, $\left(P_{n}\right)_{n \in \mathbb{N}}$ converge pontualmente a $P$ e $\left(\left\|P_{n}\right\|\right)_{n \in \mathbb{N}}$ é limitada, então $L(P ; 0)=\mathrm{K}-\lim _{n \in \mathbb{N}} L\left(P_{n} ; 0\right)$.

Demonstração. Basta notar que, nesse caso, o teorema 5.18 garante que $\lim _{n \rightarrow \infty} P_{n}\left(x_{n}\right)=P(x)$, para toda seqüência $\left(x_{n}\right)_{n \in \mathbb{N}}$ convergente a $x \in E$. Além disso, como $P$ e $\vec{P}_{n}$ são não-nulos, a proposição 5.22 nos dá o resultado.

Os resultados obtidos podem ser resumidos em um teorema global para o caso complexo:

Teorema 5.24. Sejam E um espaço de Banach complexo, $\left(P_{n}\right)_{n \in \mathbb{N}} \subset \mathcal{P}\left({ }^{k} E\right) \backslash\{0\}$ e $P \in \mathcal{P}\left({ }^{k} E\right) \backslash$ $\{0\}$. São equivalentes:

1. $P_{n} \stackrel{\mathrm{K}}{\longrightarrow} P$;

2. $L(P ; 1)=\mathrm{K}-\lim _{n \in \mathbb{N}} L\left(P_{n} ; 1\right)$;

3. A seqüência $\left(P_{n}\left(x_{n}\right)\right)_{n \in \mathbb{N}}$ converge a $P(x)$, para toda seqüência $\left(x_{n}\right)_{n \in \mathbb{N}}$ convergente a $x \in E$;

4. A seqüência $\left(P_{n}\right)_{n \in \mathbb{N}}$ converge pontualmente a $P$ e $\left(\left\|P_{n}\right\|\right)_{n \in \mathbb{N}}$ é limitada.

O exemplo seguinte mostra que, no caso real, não se pode obter um resultado tão interessante:

Exemplo 5.25. Sejam $E=\mathbb{R}^{2}, k=2, P_{n}(x, y)=x^{2}+\frac{y^{2}}{n}$ e $P(x, y)=x^{2}$.

Temos $\left\|P_{n}\right\|=\|P\|=1$, e $\lim _{n \rightarrow \infty} P_{n}(x, y)=P(x, y), \forall(x, y) \in E . \operatorname{Mas} L\left(P_{n} ; 0\right)=\{(0,0)\} \mathrm{e}$ $L(P ; 0)=\{(0, y): y \in \mathbb{R}\}$, donde $L(P ; 0) \neq \mathrm{K}-\lim _{n \in \mathbb{N}} L\left(P_{n} ; 0\right)$. 
No entanto, a adição de certas hipóteses nos permite obter um resultado semelhante no caso real. Comecemos com o seguinte:

Lema 5.26. Sejam $\left(P_{n}\right)_{n \in \mathbb{N}}$ uma seqüência de polinômios reais e $P$ um polinômio real tais que, para todo intervalo $I \subset \mathbb{R},\left(P_{n}\right)_{n \in \mathbb{N}}$ converge uniformemente a $P$ em $I, P(0)=0$ e $P^{\prime}(0) \neq 0$. Então, existe uma seqüência $\left(\lambda_{n}\right)_{n \in \mathbb{N}} \subset \mathbb{R}$, com $\lambda_{n} \rightarrow 0$, e existe $n_{0} \in \mathbb{N}$ tal que $P_{n}\left(\lambda_{n}\right)=0, \forall n>n_{0}$.

Demonstração. Podemos supor, sem perda de generalidade, que $P^{\prime}(0)>0$. Nesse caso, existe $\delta>0$ tal que, se $0<x<\delta$, então $P(x)>0$ e, se $-\delta<x<0$, então $P(x)<0$.

Por hipótese, $\left(P_{n}\right)_{n \in \mathbb{N}}$ converge uniformemente a $P$ em $(0, \delta)$. Em particular, fixado $x_{1} \in(0, \delta)$, temos que $P_{n}\left(x_{1}\right) \rightarrow P\left(x_{1}\right)$. Logo, existe $n_{1} \in \mathbb{N}$ tal que $n>n_{1} \Rightarrow P_{n}\left(x_{1}\right)>0$. Analogamente, se fixarmos $x_{2} \in(-\delta, 0)$, existe $n_{2} \in \mathbb{N}$ tal que $n>n_{2} \Rightarrow P_{n}\left(x_{2}\right)<0$.

Seja $n_{0}=\max \left\{n_{1}, n_{2}\right\}$. Então, se $n>n_{0}$, podemos afirmar que $P_{n}$ tem ao menos uma raiz no intervalo $(-\delta, \delta)$. Definamos a seqüência $\left(\lambda_{n}\right)_{n \in \mathbb{N}}$ da seguinte forma: se $n \leq n_{0}$, então $\lambda_{n}=0$. Se $n>n_{0}, \lambda_{n}$ é a raiz de $P_{n}$ mais próxima de 0 no intervalo $(-\delta, \delta)$. Observe que podemos definir a raiz mais próxima, pois o número de raízes é finito. Segue que $P_{n}\left(\lambda_{n}\right)=0, \forall n \in \mathbb{N}$.

Além disso, $\lambda_{n} \rightarrow 0$. Suponha, por absurdo, que isso não acontece. Então, existe $\epsilon>0$ tal que $\forall N \in \mathbb{N}$ existe $n>N$ com $\left|\lambda_{n}\right| \geq \epsilon$. Se $\epsilon>\delta$, a contradição é clara. Caso contrário, escolhamos $M \in \mathbb{N}$ tal que $\frac{\delta}{M}<\epsilon$. Utilizando o mesmo argumento, pode-se encontrar $n_{0}^{\prime} \in \mathbb{N}$ tal que, se $n>n_{0}^{\prime}$, então $P_{n}$ possui ao menos uma raiz em $\left(\frac{-\delta}{M}, \frac{\delta}{M}\right)$, o que contradiz a afirmação anterior.

A proposição seguinte nos apresenta o resultado procurado.

Proposição 5.27. Sejam $E$ um espaço de Banach real, $\left(P_{n}\right)_{n \in \mathbb{N}} \subset \mathcal{P}\left({ }^{k} E\right)$ e $P \in \mathcal{P}\left({ }^{k} E\right)$ com $d P(x) \not \equiv 0, \forall x \neq 0$. Se $\left(P_{n}\left(x_{n}\right)\right)_{n \in \mathbb{N}}$ converge a $P(x)$, para toda seqüência $\left(x_{n}\right)_{n \in \mathbb{N}}$ convergente a $x \in E$, então $L(P ; 0)=\mathrm{K}-\lim _{n \in \mathbb{N}} L\left(P_{n} ; 0\right)$.

Demonstração. Sejam $\left(x_{n}\right)_{n \in \mathbb{N}}$ uma seqüência em $E$ e $\left(x_{n_{k}}\right)_{k \in \mathbb{N}}$ uma subseqüência de $\left(x_{n}\right)_{n \in \mathbb{N}}$ tal que $P_{n_{k}}\left(x_{n_{k}}\right)=0, \forall k \in \mathbb{N}$, e $\left(x_{n_{k}}\right)_{k \in \mathbb{N}}$ é convergente a $x \in E$. Por hipótese, segue que $P(x)=0$. Logo, Ls $L\left(P_{n} ; 0\right) \subseteq L(P ; 0)$.

Para provar que $L(P ; 0) \subseteq \mathrm{Li} L\left(P_{n} ; 0\right)$, considere um ponto $x \in E$ tal que $P(x)=0$. Se $x=0$, é claro que $x \in \operatorname{Li} L\left(P_{n} ; 0\right)$. Se não, escolhamos $y \in E$ tal que $d P(x)(y) \neq 0$, e definamos, em $\mathbb{R}$ :

$$
f_{n}(r)=P_{n}(x+r y) \quad \text { e } \quad f(r)=P(x+r y) .
$$

Então, $f^{\prime}(r)=d P(x+r y) y \Rightarrow f^{\prime}(0)=d P(x)(y) \neq 0$. Mas $f_{n}$ e $f$ são polinômios de grau $k$ sobre $\mathbb{R}$ e $f_{n}$ converge pontualmente a $f$, por hipótese. Logo, $f_{n}$ converge a $f$ uniformemente em subconjuntos limitados. 
Pelo lema 5.26, pode-se afirmar que existe uma seqüência $\left(\lambda_{n}\right)_{n \in \mathbb{N}} \subset \mathbb{R}$ de raízes de $f_{n}$ convergindo a 0 . Dessa forma, $x+\lambda_{n} y \in L\left(P_{n} ; 0\right)$ e $\lim _{n \rightarrow \infty}\left(x+\lambda_{n} y\right)=x$, o que conclui a demonstração.

A mesma demonstração é utilizada no seguinte:

Teorema 5.28. Sejam $E$ um espaço de Banach real, $\left(P_{n}\right)_{n \in \mathbb{N}} \subset \mathcal{P}\left({ }^{k} E\right), P \in \mathcal{P}\left({ }^{k} E\right)$ e $\alpha \in \mathbb{R}$ tal que $P(x)=\alpha \Rightarrow d P(x) \not \equiv 0$. Se $\left(P_{n}\left(x_{n}\right)\right)_{n \in \mathbb{N}}$ converge a $P(x)$, para toda seqüência $\left(x_{n}\right)_{n \in \mathbb{N}}$ convergente a $x \in E$, então $L(P ; \alpha)=\mathrm{K}-\lim _{n \in \mathbb{N}} L\left(P_{n} ; \alpha\right)$.

A proposição seguinte apresenta um resultado sobre o comportamento do fecho dos conjuntos obtidos a partir da aplicação de um polinômio em uma bola aberta.

Proposição 5.29. Se $\left(P_{n}\right)_{n \in \mathbb{N}}$ e $P$ são polinômios homogêneos em um espaço de Banach $E$, $P_{n} \stackrel{K}{\longrightarrow} P$ e $B \subseteq E$ é uma bola aberta, cntão $\overline{P(B)} \subseteq \mathrm{Li} \overline{P_{n}(B)}$.

Demonstração. Seja $r \in P(B)$. Então, existe $x \in B$ tal que $P(x)=r$. Pela proposição 2.16, existe $\left(x_{n}\right)_{n \in \mathbb{N}} \subseteq E$, com $x_{n} \rightarrow x$, tal que $P_{n}\left(x_{n}\right)=r, \forall n \in \mathbb{N}$.

Além disso, podemos assumir que $\left(x_{n}\right)_{n \in \mathbb{N}} \subseteq B$, uma vez $x_{n} \in B$, para $n$ suficientemente grande. Então, $r \in P_{n}(B), \forall n \in \mathbb{N}$ e, conseqüentemente, $r \in \mathrm{Li} \overline{P_{n}(B)}$. Como Li $\overline{P_{n}(B)}$ é fechado, concluímos que $\overline{P(B)} \subseteq \mathrm{Li} \overline{P_{n}(B)}$.

Observação 5.30. Cabem as seguintes observações:

a) De acordo com a demonstração, nota-se que a homogeneidade não é imprescindivel: a continuidade seria suficiente. Além disso, o resultado é válido para qualquer bola $B \subseteq E$;

b) Se $P \not \equiv 0$, seria suficiente supor que $L(P ; \alpha)=\mathrm{K}-\lim _{n \in \mathbb{N}} L\left(P_{n} ; \alpha\right), \forall \alpha \neq 0$, pois poderíamos mostrar que $P(B) \backslash\{0\} \subseteq \mathrm{Li} \overline{P_{n}(B)}$. Então, basta notar que $\overline{P(B)}=\overline{P(B) \backslash\{0\}}$, uma vez que 0 não é um ponto isolado, já que $P \not \equiv 0$.

O exemplo seguinte mostra que a convergência no sentido de Kuratowski não é suficiente para garantir a igualdade dos conjuntos em questão:

Exemplo 5.31. Sejam $E=c_{0}(\mathbb{K}), P_{n}(x)=e_{1}^{\star}(x)^{2}-e_{n}^{\star}(x)^{2}, \forall n \in \mathbb{N}$ e $P(x)=e_{1}^{\star}(x)^{2}$.

É fácil ver que:

- $\left(P_{n}\right)_{n \in \mathbb{N}}$ converge pontualmente a $P$;

- $\|P\|=1$ e $\left\|P_{n}\right\|=1$, se $\mathbb{K}=\mathbb{R}$, ou $\left\|P_{n}\right\|=2$, se $\mathbb{K}=\mathbb{C}$. 
Pelo teorema $5.24, P_{n} \stackrel{\mathrm{K}}{\longrightarrow} P$, se $\mathbb{K}=\mathbb{C}$. Se $\mathbb{K}=\mathbb{R}$, devemos ter $L(P ; 0)=\mathrm{K}-\lim _{n \in \mathbb{N}} L\left(P_{n} ; 0\right)$. Vamos mostrar isso:

i) Se $x \in L(P ; 0)$, então $e_{1}^{\star}(x)=0$. Seja $x_{n}=x-e_{n}^{\star}(x) e_{n}$. É claro que $P_{n}\left(x_{n}\right)=0, \forall n \in \mathbb{N}$. Além disso:

$$
\left\|x_{n}-x\right\|=\left\|x-e_{n}^{\star}(x) e_{n}-x\right\| \leq \mid e_{n}^{\star}(x) \stackrel{n \rightarrow \infty}{\longrightarrow} 0 .
$$

ii.) Se $\lim _{j \rightarrow \infty} x_{n_{j}}=x$ e $x_{n_{j}} \in L\left(P_{n_{j}} ; 0\right), \forall j \in \mathbb{N}$, temos:

$$
\left\|x_{n_{j}}-x\right\| \geq\left|e_{1}^{\star}\left(x_{n_{j}}\right)-e_{1}^{\star}(x)\right|=\left|e_{1}^{\star}(x) \pm e_{n_{j}}^{\star}\left(x_{n_{j}}\right)\right| \geq\left|e_{1}^{\star}(x)\right|-\left|e_{n_{j}}^{\star}\left(x_{n_{j}}\right)\right| .
$$

A equação central é conseqüência do fato de que $P_{n_{j}}\left(x_{n_{j}}\right)=0, \forall j \in \mathbb{N}$. Então:

$$
\begin{gathered}
\left|e_{1}^{\star}(x)\right| \leq\left\|x_{n_{j}}-x\right\|+\left|e_{n_{j}}^{\star}\left(x_{n_{j}}\right)\right| \leq\left\|x_{n_{j}}-x\right\|+\left|e_{n_{j}}^{\star}\left(x_{n_{j}}-x\right)\right|+\left|e_{n_{j}}^{\star}(x)\right| \leq \\
\leq 2\left\|x_{n_{j}}-x\right\|+\left|e_{n_{j}}^{\star}(x)\right| .
\end{gathered}
$$

Fazendo $n_{j} \rightarrow+\infty$, a expressão da direita vai a zero e, conseqüentemente, $e_{1}^{\star}(x)=0$, donde $x \in L(P ; 0)$. Finalmente, $P_{n} \stackrel{\mathrm{K}}{\longrightarrow} P$, para $\mathbb{K}=\mathbb{R}$ ou $\mathbb{K}=\mathbb{C}$.

Agora, seja $B=B\left(e_{1}, \frac{1}{2}\right)$. No caso real, temos:

$$
\overline{P(B)}=\left[\frac{1}{4}, \frac{9}{4}\right] \quad \text { e } \quad \overline{P_{n}(B)}=\left[0, \frac{9}{4}\right] .
$$

Logo, Ls $\overline{P_{n}(B)} \not \subset \overline{P(B)}$. No caso complexo, $P(B)=\left\{w^{2}: w \in D\right\}$, onde $D=B\left(1 ; \frac{1}{2}\right)$. Por outro lado, $P_{n}(B)=h(A)$, onde $h: \mathbb{C} \longrightarrow \mathbb{C}$ é dada por $h\left(z_{1}, z_{2}\right)=z_{1}^{2}-z_{2}^{2} \mathrm{e}$

$$
A=\left\{\left(z_{1}, z_{2}\right) \in \mathbb{C}^{2}: z_{1} \in B\left(1 ; \frac{1}{2}\right), z_{2} \in B\left(0 ; \frac{1}{2}\right)\right\} .
$$

Claramente, Ls $\overline{P_{n}(B)} \not \subset \overline{P(B)}$, pois $\left[0, \frac{5}{2}\right] \subset \Re\left(P_{n}(B)\right), \forall n \in \mathbb{N}$, mas $\left[0, \frac{5}{2}\right] \not \subset \Re(P(B))$.

Observação 5.32. No caso real, esse exemplo diz que $\lim _{n \rightarrow \infty}\left\|P_{n}\right\|=\|P\|$, e a convergência no sentido de Kuratowski não é suficiente para provar que $\frac{n \rightarrow \infty}{P(B)}=\mathrm{K}-\lim _{n \in \mathbb{N}} \overline{P_{n}(B)}$. Em particular, o exemplo mostra que:

$$
\lim _{n \rightarrow \infty} P_{n}(x)=P(x), \forall x \in E \text { e } \lim _{n \rightarrow \infty}\left\|P_{n}\right\|=\|P\| \not \overline{P(B)}=\mathrm{K}-\lim _{n \in \mathbb{N}} \overline{P_{n}(B)} .
$$

No entanto, vale o seguinte resultado:

Proposição 5.33. Sejam $E$ um espaço de Banach, $\left(P_{n}\right)_{n \in \mathbb{N}}$ uma seqüência de polinômios homogêneos em $E$ e $P$ um polinômio homogêneo em $E$ tais que $P_{n} \stackrel{\mathrm{K}}{\longrightarrow} P$. Então, Ls $P_{n}(K) \subseteq P(K)$, para todo subconjunto compacto $K \subset E$. 
Demonstração. Se $r \in \operatorname{Ls} P_{n}(K)$, existe uma seqüência $\left(r_{n_{j}}\right)_{j \in \mathbb{N}}$ convergente a $r$ tal que $r_{n_{j}} \in$ $P_{n_{j}}(K), \forall j \in \mathbb{N}$, por definição de limite superior. Então, existe $\left(x_{n_{j}}\right)_{j \in \mathbb{N}} \in K$ tal que $P_{n_{j}}\left(x_{n_{j}}\right)=r_{n_{j}}$, donde $\lim _{j \rightarrow \infty} P_{n_{j}}\left(x_{n_{j}}\right)=r$.

Como $K$ é compacto, existe uma subseqüência de $\left(x_{n_{j}}\right)_{j \in \mathbb{N}}$ convergente a um ponto $x \in K$. Seja $\left(x_{n_{j_{l}}}\right)_{l \in \mathbb{N}}$ essa subseqüêencia. Pela proposição 5.10,

$$
P(x)=\lim _{l \rightarrow \infty} P_{n_{j_{l}}}\left(x_{n_{j_{l}}}\right)=r
$$

e $r \in P(K)$.

\subsection{Convergência no sentido de Wijsman}

Essa seção será dedicada a estabelecer relações entre convergência de polinômios e a convergência, no sentido de Wijsman, de seus conjuntos de nível.

O primeiro resultado que apresentamos é análogo ao lema 3.9, que usa fortemente a linearidade em sua demonstração, e não é válido para polinômios em geral. No entanto, temos o seguinte:

Lema 5.34. Seja $E$ um espaço de Banach complexo. Se $P \in \mathcal{P}\left({ }^{k} E\right), P \not \equiv 0$ e $k \in \mathbb{N}$, temos:

$$
d(0 ; L(P ; \alpha))=\left(\frac{|\alpha|}{\|P\|}\right)^{\frac{1}{k}}
$$

Demonstração. Sabemos que:

$$
d(0 ; L(P ; \alpha))=\inf _{x \in L(P ; \alpha)}\|x\| .
$$

Além disso, $|P(x)| \leq\|P\|\|x\|^{k}$. Logo, se $x \in L(P ; \alpha)$, então:

$$
\|x\|^{k} \geq \frac{|P(x)|}{\|P\|}=\frac{|\alpha|}{\|P\|}
$$

Mas então:

$$
\|x\| \geq\left(\frac{|\alpha|}{\|P\|}\right)^{\frac{1}{k}}, \forall x \in L(P ; \alpha) .
$$

Logo, $d(0 ; L(P ; \alpha)) \geq\left(\frac{\lfloor\alpha\rfloor}{\|P\|}\right)^{\frac{1}{k}}$.

Por outro lado, $\forall \epsilon>0$, existe $x \in E$ tal que $|P(x)|>(\|P\|-\epsilon)\|x\|^{k}$. Seja $z=\left(\frac{\alpha}{P(x)}\right)^{\frac{1}{k}} x$. É fácil ver que $z \in L(P ; \alpha)$. Além disso,

$$
\|z\|=\left|\frac{\alpha}{P(x)}\right|^{\frac{1}{k}}\|x\|<\left|\frac{\alpha}{\|P\|-\epsilon}\right|^{\frac{1}{k}} .
$$


Como $z \in L(P ; \alpha)$, temos:

$$
d(0 ; L(P ; \alpha)) \leq\left(\frac{|\alpha|}{\|P\|-\epsilon}\right)^{\frac{1}{k}}
$$

Fazendo $\epsilon \rightarrow 0$, segue o resultado.

No caso real, o lema continua válido se supusermos $k$ ímpar. Se $k$ é par, o resultado não é verdadeiro, como mostra o seguinte exemplo:

Exemplo 5.35. Sejam $E=\mathbb{R}^{2}, P(x, y)=-x^{2}+\frac{y^{2}}{4}, \alpha=\frac{1}{4}$. Nesse caso, temos:

$$
L(P ; \alpha)=\left\{(x, y) \in \mathbb{R}^{2}:-x^{2}+\frac{y^{2}}{4}=\frac{1}{4}\right\}=\left\{(x, y) \in \mathbb{R}^{2}: y^{2}=1+4 x^{2}\right\} .
$$

A partir da equação, é fácil ver que $d(0 ; L(P ; \alpha))=1$. Mas:

$$
\left(\frac{|\alpha|}{\|P\|}\right)^{\frac{1}{k}}=\left(\frac{1}{4}\right)^{\frac{1}{2}}=\frac{1}{2}
$$

A proposição seguinte analisa o que ocorre com a imagem de bolas quando assumimos a convergência, no sentido de Wijsman, dos conjuntos de nível dos polinômios. Aqui, cabe lembrar que a convergência no sentido de Wijsman é mais forte que a convergência no sentido de Kuratowski, o que explica a diferença entre a proposição seguinte e a proposição 5.33.

Proposição 5.36. Sejam $\left(P_{n}\right)_{n \in \mathbb{N}} \subset \mathcal{P}\left({ }^{k} E\right)$ e $P \in \mathcal{P}\left({ }^{k} E\right) . \quad S e L(P ; \alpha)=\mathrm{W}-\lim _{n \in \mathbb{N}} L\left(P_{n} ; \alpha\right)$, $\forall \alpha \neq 0$, então $\overline{P(B)}=\mathrm{K}-\lim _{n \in \mathbb{N}} \overline{P_{n}(B)}$, para toda bola $B \subset E$.

Demonstração. Se $P \equiv 0$, a condição $L(P ; \alpha)=\mathrm{W}-\lim _{n \in \mathbb{N}} L\left(P_{n} ; \alpha\right)$ significa que $\left(L\left(P_{n} ; 1\right)\right)_{n \in \mathbb{N}}$ converge, no sentido de Wijsman, a $\emptyset$. Equivalentemente, pode-se afirmar que:

$$
\forall x \in E, \lim _{n \rightarrow \infty} d\left(x, L\left(P_{n} ; 1\right)\right)=+\infty .
$$

Em particular, $\lim _{n \rightarrow \infty} d\left(0, L\left(P_{n} ; 1\right)\right)=+\infty$. Vamos mostrar que $\lim _{n \rightarrow \infty}\left\|P_{n}\right\|=0$.

Caso I: $\mathbb{K}=\mathbb{R}$.

Dado $M>0$, existe $n_{0} \in \mathbb{N}$ tal que $d\left(0, L\left(P_{n} ; 1\right)\right) \geq M$, se $n>n_{0}$. Logo, se $\left|P_{n}(x)\right|=1$, temos que $\|x\| \geq M$. Dado $\epsilon>0$, seja $n_{1} \in \mathbb{N}$ tal que:

$$
d\left(0, L\left(P_{n} ; 1\right)\right) \geq \frac{1}{\epsilon^{\frac{1}{k}}} .
$$


Seja $x \in B(0,1)$ tal que $P_{n}(x) \neq 0$. Então:

$$
P_{n}\left(\frac{x}{\left|P_{n}(x)\right|^{\frac{1}{k}}}\right)=\frac{P_{n}(x)}{\left|P_{n}(x)\right|}= \pm 1 .
$$

Logo, se $n>n_{1}$ :

$$
\left\|\frac{x}{\left|P_{n}(x)\right|^{\frac{1}{k}}}\right\| \geq \frac{1}{\epsilon^{\frac{1}{k}}}
$$

ou seja, $\left|P_{n}(x)\right| \leq \epsilon$, donde $\left\|P_{n}\right\| \leq \epsilon$, se $n>n_{1}$.

Caso II: $\mathbb{K}=\mathbb{C}$.

Dado $x \in B(0,1)$, seja $\lambda_{n} \in D(0,1)$ tal que $\lambda_{n}\left|P_{n}(x)\right|=P_{n}(x)$. Seja $u_{n}$ uma raiz $k$-ésima de $\lambda_{n}$. Então:

$$
P_{n}\left(\frac{x}{u_{n}\left|P_{n}(x)\right|^{\frac{1}{k}}}\right)=\frac{P_{n}(x)}{\lambda_{n}\left|P_{n}(x)\right|}=1
$$

donde segue que:

$$
\left\|\frac{x}{u_{n}\left|P_{n}(x)\right|^{\frac{1}{k}}}\right\| \geq \frac{1}{\epsilon^{\frac{1}{k}}} .
$$

Usando o mesmo raciocínio do caso anterior, segue o resultado. Logo, $\lim _{n \rightarrow \infty}\left\|P_{n}\right\|=0$. Portanto, $\mathrm{K}-\lim _{n \in \mathbb{N}} \overline{P_{n}(B)}=\{0\}=\overline{P(B)}$, para toda bola $B \subset E$, uma vez que $\left(P_{n}\right)_{n \in \mathbb{N}}$ converge uniformemente a 0 em $B$.

Suponhamos, agora, que $P \not \equiv 0$. Como a convergência no sentido de Wijsman implica a convergência no sentido de Kuratowski, a proposição 5.29 (veja a observação) assegura que precisamos mostrar somente que Ls $\overline{P_{n}(B)} \subset \overline{P(B)}$, ou, de maneira equivalente, que se $w_{n_{j}} \in \overline{P_{n_{j}}(B)}$ e $w=\lim _{j \rightarrow \infty} w_{n_{j}}$, então $w \in \overline{P(B)}$.

Consideremos $B=B\left(x_{0}, r_{0}\right)$ e assumamos, sem perda de generalidade, que $w_{n} \in P_{n}(B)$, e que $w=\lim _{n \rightarrow \infty} w_{n}$.

\section{Caso I: $w \neq 0$.}

Se $w \notin \overline{P(B)}$, afirmamos que existe $\eta>0$ tal que $d\left(x_{0}, L(P ; w)\right)>r_{0}+\eta$. De fato, seja $\epsilon>0$ tal que $w \notin\{z \in E: d(z, \overline{P(B)}) \leq \epsilon\}$. Sejam $z \in B\left(x_{0}, r_{0}+\eta\right)$ e $y_{z} \in B$ tais que $\left\|y_{z}-z\right\|<\eta$. Então:

$$
\left|P(z)-P\left(y_{z}\right)\right| \leq M \eta
$$

onde $M$ depende apenas de $r_{0}$, de $k$ e de $\|P\|$. Escolhendo $\eta$ de forma que $M \eta<\epsilon$, a afirmação está demonstrada. 
Em função dessa afirmação, a convergência no sentido de Wijsman nos permite assumir que $d\left(x_{0}, L\left(P_{n} ; w\right)\right)>r_{0}+\eta, \forall n \in \mathbb{N}$. Escolhamos, agora, $z_{n} \in B$ tal que $P_{n}\left(z_{n}\right)=w_{n}$, e definamos $\theta_{n}=\left(\frac{w}{w_{n}}\right)^{\frac{1}{k}}$. Temos:

$$
P_{n}\left(\theta_{n} z_{n}\right)=P_{n}\left(\left(\frac{w}{w_{n}}\right)^{\frac{1}{k}} z_{n}\right)=\frac{w}{w_{n}} P_{n}\left(z_{n}\right)=w
$$

donde $d\left(x_{0}, L\left(P_{n} ; w\right)\right) \leq\left\|x_{0}-\theta_{n} z_{n}\right\|$. Mas então:

$$
r_{0}+\eta \leq \lim _{n \rightarrow \infty} d\left(x_{0}, L\left(P_{n} ; w\right)\right) \leq \lim _{n \rightarrow \infty}\left\|x_{0}-\theta_{n} z_{n}\right\| \leq r_{0}
$$

pois:

$$
\left\|x_{0}-\theta_{n} z_{n}\right\|=\left\|x_{0}-z_{n}+z_{n}-\theta_{n} z_{n}\right\| \leq\left\|x_{0}-z_{n}\right\|+\left|1-\theta_{n}\right|\left\|z_{n}\right\|<r_{0}+\left|1-\theta_{n}\right|\left(\left\|x_{0}\right\|+r\right)
$$

e $\theta_{n} \rightarrow 1$ quando $n \rightarrow \infty$, contradição.

Caso II: $w=0$.

Se $0 \notin \overline{P(B)}$, existe $\epsilon>0$ tal que $B(0 ; \epsilon) \cap P(B)=\emptyset$. Sejam $y_{0} \in B$ e $y_{n} \in B$ tais que $P_{n}\left(y_{n}\right)=w_{n}$. Como $w_{n} \rightarrow 0$, podemos assumir, sem perda de generalidade, que $\left|w_{n}\right|<\frac{\epsilon}{2}$, para $n$ suficientemente grande.

Escolha $z_{n} \in\left[y_{0}, y_{n}\right] \subset B$ tal que $P_{n}\left(z_{n}\right) \in B(0 ; \epsilon) \backslash B\left(0 ; \frac{\epsilon}{2}\right)$. Esse ponto existe, pois:

- $P\left(y_{0}\right)=\lim _{n \rightarrow \infty} P_{n}\left(y_{0}\right)$, o que é conseqüência da convergência no sentido de Kuratowski;

- $P\left(y_{0}\right) \in P(B)$, e $P(B) \cap B(0 ; \epsilon)=\emptyset$.

Então, $\left(P_{n}\left(z_{n}\right)\right)_{n \in \mathbb{N}}$ possui uma subseqüência convergente a $w_{0} \in B(0 ; \epsilon) \backslash\{0\}$. Dessa forma, basta usar o Caso I para obtermos uma contradição, e a proposição está demonstrada.

A partir de agora, vamos utilizar a seguinte notação: fixemos $x \in E$ e $\alpha \in \mathbb{K}$, e sejam $P, P_{n} \in$ $\mathcal{P}\left({ }^{k} E\right) \backslash\{0\}$. Definamos:

$$
\lambda=d(x, L(P ; \alpha)) \quad \text { e } \quad \lambda_{n}=d\left(x, L\left(P_{n} ; \alpha\right)\right) .
$$

Como a convergência no sentido de Kuratowski é mais fraca que a convergência no sentido de Wijsman, o fato de que $P_{n} \stackrel{\mathrm{K}}{\longrightarrow} P$ não implica $\lambda=\lim _{n \rightarrow \infty} \lambda_{n}$. No entanto, o seguinte resultado é válido:

Proposição 5.37. Sejam $\left(P_{n}\right)_{n \in \mathbb{N}} \subset \mathcal{P}(E)$ e $P \in \mathcal{P}(E)$ tais que $P_{n} \stackrel{\mathrm{K}}{\rightarrow} P$. Então, $\forall \epsilon>0$ existe $n_{0} \in \mathbb{N}$ tal que $\lambda_{n}<\lambda+\epsilon, \forall n \geq n_{0}$. 
Demonstração. Seja $z \in B(x, \lambda+\epsilon) \operatorname{com} P(z)=\alpha$. Pela caracterização da convergência no sentido de Kuratowski, dada pela proposição 2.16 , existe uma seqüência $\left(z_{n}\right)_{n \in \mathbb{N}}$ convergente a $z$ tal que $P_{n}\left(z_{n}\right)=\alpha$. Logo, existe $n_{0} \in \mathbb{N}$ tal que $z_{n} \in B(x, \lambda+\epsilon), \forall n \geq n_{0}$. Segue que:

$$
\lambda_{n} \leq\left\|z_{n}-x\right\|<\lambda+\epsilon, \forall n \geq n_{0} .
$$

Corolário 5.38. Sejam $\left(P_{n}\right)_{n \in \mathbb{N}} \subset \mathcal{P}(E)$ e $P \in \mathcal{P}(E)$ tais que $P_{n} \stackrel{\mathrm{K}}{\longrightarrow} P$. Então, $\limsup _{n \rightarrow \infty} \lambda_{n} \leq \lambda$.

Demonstração. Imediata, a partir da proposição anterior.

Vamos, agora, estudar uma recíproca da proposição 5.36. Iniciaremos com o seguinte lema:

Lema 5.39. Sejam $\alpha \neq 0,\left(P_{n}\right)_{n \in \mathbb{N}} \subset \mathcal{P}(E)$ e $P \in \mathcal{P}(E)$. Se Ls $\overline{P_{n}(B)} \subseteq \overline{P(B)}$, para toda bola $B \subset E$, então $\lambda \leq \liminf _{n \rightarrow \infty} \lambda_{n}$.

Demonstração. Se a conclusão não é verdadeira, existem $\lambda^{\star}<\lambda$ e uma subseqüiência $\left(\lambda_{n_{k}}\right)_{k \in \mathbb{N}}$ de $\left(\lambda_{n}\right)_{n \in \mathbb{N}}$ tais que $\lambda_{n_{k}}<\lambda^{\star}, \forall k \in \mathbb{N}$.

Fixe $x \in E$, e seja $B=B\left(x, \lambda^{\star}\right)$. Observe que $B\left(x, \lambda_{n_{k}}\right) \subset B$. Para cada $k \in \mathbb{N}$, existe $z_{n_{k}} \in B$ tal que $P_{n_{k}}\left(z_{n_{k}}\right)=\alpha$ (ou a inclusão não seria estrita). Logo, $\alpha \in P(B), \forall k \in \mathbb{N}$, e, portanto, $\alpha \in \overline{P(B)}$. Agora, para cada $j \in \mathbb{N}$, escolhamos $y_{j} \in B$ de forma que $\left|\alpha-P\left(y_{j}\right)\right|<\frac{1}{j}$, e definamos:

$$
\gamma_{j}=\left(\frac{\alpha}{P\left(y_{j}\right)}\right)^{\frac{1}{k}}
$$

Note que, como $\alpha \neq 0$, podemos assumir que $P\left(y_{j}\right) \neq 0$ e que, no caso real, $s g\left(P\left(y_{j}\right)\right)=s g(\alpha)$. Então, $P\left(\gamma_{j} y_{j}\right)=\alpha$. Observe que:

$$
\begin{aligned}
\left\|\gamma_{j} y_{j}-x\right\|= & \left\|\gamma_{j} y_{j}-y_{j}+y_{j}-x\right\| \leq\left|\gamma_{j}-1\right|\left\|y_{j}\right\|+\left\|y_{j}-x\right\|< \\
& <\left|\gamma_{j}-1\right|\left(\|x\|+\lambda^{\star}\right)+\lambda^{\star}<\frac{\lambda+\lambda^{\star}}{2}
\end{aligned}
$$

quando $j$ é suficientemente grande. Logo, $\gamma_{j} y_{j} \in B\left(x, \frac{\lambda+\lambda^{*}}{2}\right) \subset B(x, \lambda)$ quando $j$ é suficientemente grande, o que contradiz a definição de $\lambda$.

Proposição 5.40. Sejam $\left(P_{n}\right)_{n \in \mathbb{N}} \subset \mathcal{P}\left({ }^{k} E\right)$ e $P \in \mathcal{P}\left({ }^{k} E\right)$. Se, para toda bola $B \subset E$, vale que $\mathrm{K}-\lim _{n \in \mathbb{N}} \overline{P_{n}(B)}=\overline{P(B)}$, então $L(P ; \alpha)=\mathrm{W}-\lim _{n \in \mathbb{N}} L\left(P_{n} ; \alpha\right), \forall \alpha \neq 0$.

Demonstração. Se $P \equiv 0$, o resultado segue do fato de que, se $\lim _{n \rightarrow \infty} \overline{P_{n}(B)}=\{0\}$ para qualquer bola $B \subset E$, então $\lim _{n \rightarrow \infty} d\left(x, L\left(P_{n} ; \alpha\right)\right)=+\infty$, para todo $x \in E$ e todo $\alpha \neq 0$. 
Se $P \not \equiv 0$, podemos assumir, sem perda de generalidade, que nenhum termo $P_{n}$ é identicamente nulo pois, se infinitos termos o fossem, teríamos $\overline{P_{n}(B)}=\{0\}$ para infinitos valores de $n$, o que nos daria $P \equiv 0$, absurdo.

Seja $\alpha \neq 0$, e fixe $x_{0} \in E$. Precisamos mostrar que $\lambda=\lim _{n \rightarrow \infty} \lambda_{n}$. Pelo lema 5.39, sabemos que $\lambda \leq \liminf _{n \rightarrow \infty} \lambda_{n}$. Logo, basta mostrarmos que $\forall \epsilon>0$ existe $n_{0} \in \mathbb{N}$ tal que $\lambda_{n}\left\langle\lambda+\epsilon, \forall n \geq n_{0}\right.$.

Seja $B=B\left(x_{0}, \lambda+\frac{\epsilon}{2}\right)$. Pela definição de $\lambda$, existe $z \in B$ tal que $P(z)=\alpha$, donde $\alpha \in P(B)$. Como $P(B) \subseteq \mathrm{Li} \overline{P_{n}(B)}$, por hipótese, podemos escolher uma seqüência $\left(\alpha_{n}\right)_{n \in \mathbb{N}}$ convergente a $\alpha$ tal que $\alpha_{n} \in P_{n}(B), \forall n \in \mathbb{N}$. Então, existe $\left(z_{n}\right)_{n \in \mathbb{N}} \subset B$ tal que $P_{n}\left(z_{n}\right)=\alpha_{n}, \forall n \in \mathbb{N}$. Defina:

$$
z_{n}^{\prime}=\left(\frac{\alpha}{\alpha_{n}}\right)^{\frac{1}{k}} z_{n}
$$

Então, $z_{n}^{\prime} \in L\left(P_{n} ; \alpha\right)$ e $z_{n}^{\prime} \in B\left(x_{0}, \lambda+\epsilon\right)$ para $n$ suficientemente grande, já que:

$$
\left\|z_{n}^{\prime}-z\right\|=\left|1-\left(\frac{\alpha}{\alpha_{n}}\right)^{\frac{1}{k}}\right|\left\|z_{n}\right\| \quad \text { e } \quad \alpha_{n} \rightarrow \alpha .
$$

Concluímos, portanto, que $\lambda_{n}<\lambda+\epsilon$.

A união dos resultados anteriores nos dá o seguinte:

Teorema 5.41. Sejam $\left(P_{n}\right)_{n \in \mathbb{N}} \subset \mathcal{P}\left({ }^{k} E\right)$ e $P \in \mathcal{P}\left({ }^{k} E\right)$. Então, $L(P ; \alpha)=\mathrm{W}-\lim _{n \in \mathbb{N}} L\left(P_{n} ; \alpha\right), \forall \alpha \in$ $\mathbb{K}, \alpha \neq 0 \Leftrightarrow \overline{P(B)}=\mathrm{K}-\lim _{n \in \mathbb{N}} \overline{P_{n}(B)}$, para toda bola aberta $B \subseteq E$.

Demonstração. Basta utilizar as proposições 5.36 e 5.40 .

Utilizando os teoremas 5.18 e 5.41 e o fato de que a convergência no sentido de Wijsman implica a convergência no sentido de Kuratowski, temos:

Proposição 5.42. Sejam $\left(P_{n}\right)_{n \in \mathbb{N}} \subset \mathcal{P}\left({ }^{k} E\right)$ eP $P \in \mathcal{P}\left({ }^{k} E\right)$. Se $L(P ; \alpha)=\mathrm{W}-\lim _{n \in \mathbb{N}} L\left(P_{n} ; \alpha\right), \forall \alpha \neq 0$, então $\lim _{n \rightarrow \infty} P_{n}(x)=P(x), \forall x \in E, e \lim _{n \rightarrow \infty}\left\|P_{n}\right\|=\|P\|$.

Essa proposição estabelece um paralelo com o teorema 3.10, válido para o caso de funcionais lineares contínuos. No entanto, o exemplo 5.31 mostra que a recíproca dessa proposição não é verdadeira, e também apresenta uma seqüência convergente no sentido de Kuratowski que não converge no sentido de Wijsman.

Estudemos, agora, a convergência, no sentido de Wijsman, de $\left(L\left(P_{n} ; 0\right)\right)_{n \in \mathbb{N}}$. Vamos começar com o caso complexo. Antes, porém, relembremos a seguinte notação: fixados $x \in E,\left(P_{n}\right)_{n \in \mathbb{N}} \subset$ $\mathcal{P}(E)$ e $P \in \mathcal{P}(E)$, temos, com $\alpha=0$ :

$$
\lambda_{n}(x)=d\left(x, L\left(P_{n} ; 0\right)\right) \quad \text { e } \quad \lambda(x)=d(x, L(P ; 0)) .
$$


Proposição 5.43. Sejam $E$ um espaço de Banach complexo, $\left(P_{n}\right)_{n \in \mathbb{N}} \subset \mathcal{P}\left({ }^{k} E\right) \backslash\{0\}$ e $P \in \mathcal{P}\left({ }^{k} E\right) \backslash$ $\{0\}$. Se $\overline{P(B)}=\mathrm{K}-\lim _{n \in \mathbb{N}} \overline{P_{n}(B)}$, para toda bola aberta $B \subseteq E$, então $\lim _{n \rightarrow \infty} \lambda_{n}(x)=\lambda(x), \forall x \in E$.

Demonstração. Pela proposição 5.40 , temos que $L(P ; \alpha)=\mathrm{W}-\lim _{n \in \mathbb{N}} L\left(P_{n} ; \alpha\right), \forall \alpha \neq 0$. Dessa forma, a proposição 5.42 garante que $\left(P_{n}\right)_{n \in \mathbb{N}}$ converge pontualmente a $P$ e que $\left(\left\|P_{n}\right\|\right)_{n \in \mathbb{N}}$ é limitada. Assim, pelo teorema $5.24, P_{n} \stackrel{\mathrm{K}}{\longrightarrow} P$. Pelo corolário 5.38, $\lim \sup \lambda_{n}(x) \leq \lambda(x), \forall x \in E$.

Se o resultado não é válido, existe $x \in E$ tal que $\liminf _{n \rightarrow \infty} \lambda_{n}(x)<\lambda(x)=\lambda$. Passando a uma subseqüência, se necessário, podemos assumir que $\lim _{n \rightarrow \infty}^{n \rightarrow \infty} \lambda_{n}(x)=\lambda^{\star}<\lambda^{\prime}<\lambda$. Então, $0 \in$ $P_{n}\left(B\left(x, \lambda^{\prime}\right)\right), \forall n \in \mathbb{N}$, donde $0 \in \overline{P\left(B\left(x, \lambda^{\prime}\right)\right)}$. Isso nos permite escolher uma seqüência $\left(y_{n}\right)_{n \in \mathbb{N}}$ em $B\left(x, \lambda^{\prime}\right)$ de forma que $\lim _{n \rightarrow \infty} P\left(y_{n}\right)=0$. Seja $x_{0} \in B(0,1)$ tal que $P\left(x_{0}\right) \neq 0$.

Agora, seja $\epsilon=\lambda-\lambda^{\prime}$, e defina $\phi_{n}: D(0, \epsilon) \longrightarrow \mathbb{C}$ por $\phi_{n}(w)=P\left(y_{n}+w x_{0}\right)$. Observe que:

$$
\left|\phi_{n}(w)\right|=\left|P\left(y_{n}+w x_{0}\right)\right| \leq\|P\|\left(\left\|y_{n}\right\|+|w|\left\|x_{0}\right\|\right)^{k} \leq\left(\|x\|+\lambda^{\prime}+\epsilon\right)^{k} .
$$

Logo, $\left(\phi_{n}\right)_{n \in \mathbb{N}}$ é uniformemente limitada em $D(0, \epsilon)$. Além disso, como $\phi_{n}$ é um polinômio, para cada $n \in \mathbb{N}$, a seqüência $\left(\phi_{n}\right)_{n \in \mathbb{N}}$ é eqüicontínua em cada parte compacta de $D(0, \epsilon)$. Assim, pelo teorema de Ascoli-Arzelá (vide [11], págs. 244 a 248), podemos afirmar que uma subseqüência de $\left(\phi_{n}\right)_{n \in \mathbb{N}}$ converge uniformemente a um polinômio $\phi$ em $D(0, \epsilon)$. Além disso,

$$
\phi(0)=\lim _{n \rightarrow \infty} \phi_{n}(0)=\lim _{n \rightarrow \infty} P\left(y_{n}+0 x_{0}\right)=0 .
$$

Por outro lado, $\phi_{n}$ não se anula, pois $P$ não possui zeros em $B(x, \lambda)$, pela definição de $\lambda$. Usando o corolário 5.21, concluímos que $\phi \equiv 0$, o que contradiz o fato de que $P\left(x_{0}\right) \neq 0$.

Observação 5.44. Cabem as seguintes observações:

a) Se $P \equiv 0$, a proposição 5.43 é falsa. Vamos mostrar isso. Sejam $P_{n}: \mathbb{C} \longrightarrow \mathbb{C}$, com $P_{n}(z)=\frac{z}{n}$. Temos:

- $\lambda(z)=0$, pois $P \equiv 0$ e $d(z, L(P ; 0))=d(z, \mathbb{C})=0 ;$

- $\lambda_{n}(z)=|z|$, pois $d\left(z, L\left(P_{n} ; 0\right)\right)=d(z,\{0\})=|z|$.

Mas, se $B$ é uma bola contida em $\mathbb{C}$, temos $P(B)=\{0\}$ e $P_{n}(B)=\frac{1}{n} B$. Dessa forma, segue que $\mathrm{K}-\lim _{n \in \mathbb{N}} \overline{P_{n}(B)}=\overline{P(B)}$

b) A mesma prova pode ser utilizada para estender o resultado a polinômios contínuos e não-constantes. Da condição "K- $\lim _{n \in \mathbb{N}} \overline{P_{n}(B)}=\overline{P(B)}$, para toda bola aberta $B \subseteq E$ " segue que $\left(P_{n}\left(x_{n}\right)\right)_{n \in \mathbb{N}}$ converge a $P(x)$ para toda seqüência $\left(x_{n}\right)_{n \in \mathbb{N}}$ convergente a $x$ (teorema 5.18 
e proposição 5.41). Basta, então, substituir o teorema 5.24 pela proposição 5.22 na demonstração anterior.

Essa proposição é falsa no caso real, mesmo sob hipóteses mais fortes. Vejamos o seguinte exemplo:

Exemplo 5.45. Sejam $P, P_{n}: c_{0} \longrightarrow \mathbb{R}$ definidos por:

$$
P(x)=\sum_{k=1}^{\infty} \frac{1}{k^{2}}\left(x_{k}-x_{k+1}\right)^{2} \quad \text { e } \quad P_{n}(x)=\sum_{k=1}^{n} \frac{1}{k^{2}}\left(x_{k}-x_{k+1}\right)^{2}
$$

Observe que:

- $\left(P_{n}\right)_{n \in \mathbb{N}}$ converge uniformemente a $P$ em subconjuntos limitados de $c_{0}$;

- $P(x)=0 \Leftrightarrow x=0$

- $L(P ; 0)=\{0\}$;

- $L\left(P_{n} ; 0\right)=\left\{x \in c_{0}(\mathbb{R}): x_{1}=x_{2}=\cdots=x_{n}=x_{n+1}\right\}$.

Temos que:

$$
d\left(e_{1}, L(P ; 0)\right)=1 \quad \text { e } \quad d\left(e_{1}, L\left(P_{n} ; 0\right)\right)=\inf _{x \in L\left(P_{n} ; 0\right)}\left\|e_{1}-x\right\|=\frac{1}{2} .
$$

Conseqüentemente, $\lim _{n \rightarrow \infty} d\left(e_{1}, L\left(P_{n} ; 0\right)\right) \neq d\left(e_{1}, L(P ; 0)\right)$. Mas $\mathrm{K}-\lim _{n \in \mathbb{N}} \overline{P_{n}(B)}=\overline{P(B)}$, uma vez que $\left(P_{n}\right)_{n \in \mathbb{N}}$ converge uniformemente a $P$ em $B$.

Incidentalmente, o exemplo também mostra que o seguinte resultado, verdadeiro no caso complexo, não é válido no caso real:

$$
B=B\left(x_{0}, r\right), 0 \in \overline{P(B)}, \eta>0 \Rightarrow L(P ; 0) \cap B\left(x_{0}, r+\eta\right) \neq \emptyset .
$$

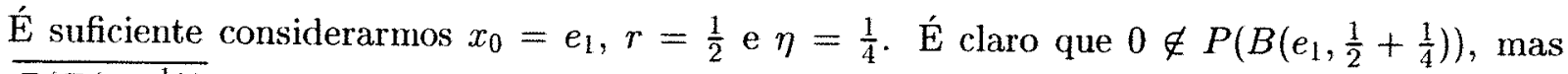
$0 \in \overline{P\left(B\left(e_{1}, \frac{1}{2}\right)\right)}$, pois:

$$
z_{n}=\left(\frac{1}{2}+\frac{1}{n}\right) e_{1}+\sum_{j=2}^{n}\left(\frac{1}{2}-\frac{1}{n}\right) e_{j} \in B\left(e_{1}, \frac{1}{2}\right) \quad \text { e } \quad 0 \leq P\left(z_{n}\right) \leq \frac{5}{n^{2}} \stackrel{n \rightarrow \infty}{\longrightarrow} 0 .
$$

Observação 5.46. Nesse exemplo, $d P(x)=0 \Leftrightarrow x=0$. Dessa forma, podemos utilizar a proposição 5.27 para mostrar que $\left(L\left(P_{n} ; 0\right)\right)_{n \in \mathbb{N}}$ converge, no sentido de Kuratowski, a $L(P ; 0)$.

Além disso, como $\mathrm{K}-\lim _{n \in \mathbb{N}} \overline{P_{n}(B)}=\overline{P(B)}$, o teorema 5.41 afirma que $L(P ; \alpha)=\mathrm{W}-\lim _{n \in \mathbb{N}} L\left(P_{n} ; \alpha\right)$, $\forall \alpha \neq 0$. Como a convergência no sentido de Wijsman implica a convergência no sentido de Kuratowski, segue que $P_{n} \stackrel{K}{\longrightarrow} P$. 
Para obtermos um resultado similar no caso real, são necessárias condições mais fortes sobre o polinômio $P$ :

Proposição 5.47. Seja $E$ um espaço de Banach real, e sejam $\left(P_{n}\right)_{n \in \mathbb{N}} \subset \mathcal{P}(E)$ e $P \in \mathcal{P}(E)$ tais que $P$ satisfaz:

$$
\lim _{n \rightarrow \infty}\left\|d P\left(y_{n}\right)\right\|=0 \Rightarrow 0 \in \overline{c o}\left(\left(y_{n}\right)_{n \in \mathbb{N}}\right),
$$

com $\overline{c o}\left(\left(y_{n}\right)_{n \in \mathbb{N}}\right)$ a envoltória convexa de $\left(y_{n}\right)_{n \in \mathbb{N}}$. Se $\mathrm{K}-\lim _{n \in \mathbb{N}} \overline{P_{n}(B)}=\overline{P(B)}$, para toda bola aberta $B \subseteq E$, então $\lim _{n \rightarrow \infty} \lambda_{n}(x)=\lambda(x), \forall x \in E$.

Demonstração. Cabe relembrar que, nesse caso:

$$
\lambda_{n}(x)=d\left(x, L\left(P_{n} ; 0\right)\right) \quad \text { e } \quad \lambda(x)=d(x, L(P ; 0))
$$

A condição sobre o polinômio $P$ nos garante que $d P(x)=0 \Leftrightarrow x=0$. De fato, se $x=0$, entâo $d P(x)=0$. Reciprocamente, suponha que $d P(x)=0$. Se tomarmos a seqüencia $y_{n}=x, \forall n \in \mathbb{N}$, temos $\lim _{n \rightarrow \infty}\left\|d P\left(y_{n}\right)\right\|=0$. Por hipótese, $0 \in \overline{c o}\left(\left(y_{n}\right)_{n \in \mathbb{N}}\right)=\overline{c o}(\{x\})=\{x\}$, e $x=0$. Pela observação 5.46, $P_{n} \stackrel{\mathrm{K}}{\longrightarrow} P$.

Analogamente ao que foi feito na proposição 5.43, se $\lim _{n \rightarrow \infty} \lambda_{n}(x) \neq \lambda(x)$, podemos obter uma seqüiência $\left(y_{n}\right)_{n \in \mathbb{N}} \subseteq B\left(x, \lambda^{\prime}\right)$, com $\lambda^{\prime}<\lambda=\lim _{n \rightarrow \infty} \lambda_{n}(x)$, tal que $\lim _{n \rightarrow \infty} P\left(y_{n}\right)=0$.

Tome $\epsilon=\lambda-\lambda^{\prime}$, e defina $\phi_{n}:[-\epsilon, \epsilon] \longrightarrow \mathbb{R}$ por $\phi_{n}(t)=P\left(y_{n}+t z_{n}\right)$, onde $\left(z_{n}\right)_{n \in \mathbb{N}}$ são pontos da bola unitária para os quais vale:

$$
\left|d P\left(y_{n}\right)\left(z_{n}\right)\right|>\frac{1}{2}\left\|d P\left(y_{n}\right)\right\| .
$$

Observe que esses polinômios nunca se anulam e que, como $P\left(y_{n}\right) \rightarrow 0,\left(\phi_{n}\right)_{n \in \mathbb{N}}$ converge a um polinômio $\phi$ tal que $\phi(0)=0$. Pelo lema 5.26, necessariamente temos $\phi^{\prime}(0)=0$, donde:

$$
\lim _{n \rightarrow \infty} d P\left(y_{n}\right)\left(z_{n}\right)=\lim _{n \rightarrow \infty} \phi_{n}^{\prime}(0)=\phi^{\prime}(0)=0
$$

Segue que $\lim _{n \rightarrow \infty}\left\|d P\left(y_{n}\right)\right\|=0$, absurdo, pois $0 \notin B(x, \lambda)$ e $\overline{c o}\left(\left(y_{n}\right)_{n \in \mathbb{N}}\right) \subseteq B(x, \lambda)$.

\section{4 r-Convergência}

Nessa seção, discutiremos as relações das convergências de polinômios com a r-convergência de seus conjuntos de nível. O principal objetivo dessa seção é demonstrar um resultado que generalize o teorema 3.11 . 
Relembremos, aqui, duas notações que serão largamente utilizadas ao longo da seção: fixados $x \in E, \alpha \in \mathbb{K},\left(P_{n}\right)_{n \in \mathbb{N}} \subset \mathcal{P}(E)$ e $P \in \mathcal{P}(E)$, temos

$$
\lambda=d(x, L(P ; \alpha)) \quad \text { e } \quad \lambda_{n}=d\left(x, L\left(P_{n} ; \alpha\right)\right) .
$$

O lema seguinte demonstra a suficiência da r-convergência dos conjuntos de nível para a convergência uniforme em subconjuntos limitados de uma seqüência de polinômios homogêneos. Como na proposição 5.36 , o resultado é válido se $P \equiv 0$.

Lema 5.48. Sejam $E$ um espaço de Banach, $\left(P_{n}\right)_{n \in \mathbb{N}} \subset \mathcal{P}\left({ }^{k} E\right) \backslash\{0\}$ e $P \in \mathcal{P}\left({ }^{k} E\right) \backslash\{0\}$. Se $L(P ; \alpha)=\mathrm{r}-\lim _{n \in \mathbb{N}} L\left(P_{n} ; \alpha\right), \forall \alpha \neq 0$, então $\left(P_{n}\right)_{n \in \mathbb{N}}$ converge a $P$ uniformemente em conjuntos limitados.

Demonstração. Se não ocorre essa convergência, existem $\epsilon>0$, uma bola $B \subseteq E$ e uma seqüiencia $\left(x_{n}\right)_{n \in \mathbb{N}} \subseteq B$ tais que $\left|P\left(x_{n}\right)-P_{n}\left(x_{n}\right)\right|>\epsilon, \forall n \in \mathbb{N}$ (pode-se passar a uma subsequiência, se necessário). Seja $\gamma_{n}=P\left(x_{n}\right)$. Como $\left(\gamma_{n}\right)_{n \in \mathbb{N}}$ é limitada (pois $\left(x_{n}\right)_{n \in \mathbb{N}} \subseteq B$ ), podemos assumir (se necessário, utilizando uma subseqüência) que $\gamma_{n} \rightarrow \gamma$.

Caso I: $\gamma \neq 0$.

Sem perda de generalidade, podemos assumir que $\frac{1}{2}<\left|\frac{\gamma}{\gamma_{n}}\right|<2$, ou, no caso real, que $\frac{1}{2}<\frac{\gamma}{\gamma_{n}}<2$. Fixemos $x \in E$, e definamos $\lambda=d(x, L(P ; \gamma))$ e $\lambda_{n}=d\left(x, L\left(P_{n} ; \gamma\right)\right)$. A r-convergência nos garante que $\left(\lambda_{n}\right)_{n \in \mathbb{N}}$ converge a $\lambda$ uniformemente em conjuntos limitados (vide proposição 2.27).

Seja $x_{n}^{\prime}=\left(\frac{\gamma}{\gamma_{n}}\right)^{\frac{1}{k}} x_{n}$. Note que $x_{n}^{\prime} \in 2 B$. Temos:

$$
\left|\gamma-P_{n}\left(x_{n}^{\prime}\right)\right|=\left|\frac{\gamma}{\gamma_{n}}\left(P_{n}\left(x_{n}\right)-P\left(x_{n}\right)\right)\right|=\left|\frac{\gamma}{\gamma_{n}}\right|\left|P_{n}\left(x_{n}\right)-P\left(x_{n}\right)\right|>\frac{\epsilon}{2} .
$$

Por outro lado, $\left|\lambda_{n}\left(x_{n}^{\prime}\right)\right|=\left|\lambda_{n}\left(x_{n}^{\prime}\right)-\lambda\left(x_{n}^{\prime}\right)\right|$ e então, pela convergência uniforme de $\left(\lambda_{n}\right)_{n \in \mathbb{N}}$ em $2 B$, segue que $\forall \eta>0 \exists n_{0} \in \mathbb{N}$ tal que $\left|\lambda_{n}\left(x_{n}^{\prime}\right)\right|<\eta, \forall n>n_{0}$. Essa condição pode ser reescrita como:

$$
\forall \eta>0 \exists z_{n}^{\prime} \in L\left(P_{n} ; \lambda\right) \text { tal que }\left\|z_{n}^{\prime}-x_{n}^{\prime}\right\|<\eta
$$

Então:

$$
\left|\gamma-P_{n}\left(x_{n}^{\prime}\right)\right|=\left|P_{n}\left(z_{n}^{\prime}\right)-P_{n}\left(x_{n}^{\prime}\right)\right| \leq M \eta<\frac{\epsilon}{2},
$$

se começarmos com $\eta<\frac{\epsilon}{2 M}$, onde $M$ é uma constante dependendo somente de $B$, de $k$ e de $\left\|P_{n}\right\|$, que é uniformemente limitada, pela proposição 5.10 . Isso contradiz a primeira equação.

Caso II: $\gamma=0$. 
Isso significa que $\lim _{n \rightarrow \infty} P\left(x_{n}\right)=0$. Consequentemente, $\left|P_{n}\left(x_{n}\right)\right|>\frac{\epsilon}{2}$, para $n$ suficientemente grande. Como $\left(P_{n}\left(x_{n}\right)\right)_{n \in \mathbb{N}}$ é limitado, podemos assumir, passando a uma subseqüência se necessário, que $\beta=\lim _{n \rightarrow \infty} P_{n}\left(x_{n}\right), \operatorname{com} \beta \neq 0$. Se denotarmos $\beta_{n}=P_{n}\left(x_{n}\right)$, podemos assumir que $\frac{1}{2}<\left|\frac{\beta}{\beta_{n}}\right|<2$, ou, no caso real, que $\frac{1}{2}<\frac{\beta}{\beta_{12}}<2$. Então:

$$
\left|P\left(x_{n}^{\prime}\right)-\beta\right|=\left|\frac{\beta}{\beta_{n}}\left(P\left(x_{n}\right)-P_{n}\left(x_{n}\right)\right)\right|>\frac{\epsilon}{2}
$$

onde $x_{n}^{\prime}=\left(\frac{\beta}{\beta_{n}}\right)^{\frac{1}{k}} x_{n} \in 2 B \cap L\left(P_{n} ; \beta\right)$. Se definirmos:

$$
d(x)=d(x, L(P ; \beta)) \quad \text { e } \quad d_{n}(x)=d\left(x, L\left(P_{n} ; \beta\right)\right)
$$

podemos afirmar que existe $n_{0} \in \mathbb{N}$ tal que

$$
\left|d\left(x_{n}^{\prime}\right)\right|=\left|d\left(x_{n}^{\prime}\right)-d_{n}\left(x_{n}^{\prime}\right)\right|<\frac{\epsilon}{2 M}, \forall n>n_{0},
$$

pois $d_{n}\left(x_{n}^{\prime}\right)=0$ e $\left(d_{n}\right)_{n \in \mathbb{N}}$ converge uniformemente a $d$ em $2 B$. A constante $M$ depende apenas de $B$, de $k$ e de $\|P\|$. Então, existe $z_{n}^{\prime} \in L(P ; \beta)$ tal que $\left\|z_{n}^{\prime}-x_{n}^{\prime}\right\|<\frac{\epsilon}{2 M}$ e, consequentemente:

$$
\left|P\left(x_{n}^{\prime}\right)-\beta\right|=\left|P\left(x_{n}^{\prime}\right)-P\left(z_{n}^{\prime}\right)\right|<M \frac{\epsilon}{2 M}=\frac{\epsilon}{2},
$$

o que gera uma contradição.

Mostremos, agora, a recíproca desse resultado. $O$ caso $P \equiv 0$ é tratado de forma identica ao que foi feito quando do estudo da convergência no sentido de Wijsman.

Lema 5.49. Sejam $E$ um espaço de Banach, $\left(P_{n}\right)_{n \in \mathbb{N}} \subset \mathcal{P}\left({ }^{k} E\right) \backslash\{0\}$ e $P \in \mathcal{P}\left({ }^{k} E\right) \backslash\{0\}$. Se $\left(P_{n}\right)_{n \in \mathbb{N}}$ converge a $P$ uniformemente em subconjuntos limitados, então $L(P ; \alpha)=\mathrm{r}-\lim _{n \in \mathbb{N}} L\left(P_{n} ; \alpha\right), \forall \alpha \neq 0$.

Demonstração. Fixemos $\alpha \neq 0$, e sejam $\lambda, \lambda_{n}$ definidos como anteriormente. De acordo com a proposição 2.27 , precisamos mostrar que $\left(\lambda_{n}\right)_{n \in \mathbb{N}}$ converge a $\lambda$ uniformemente em subconjuntos limitados de $E$. Fixemos $B=B(0, r)$ e $\epsilon \in(0,1)$. Pela definição de $\lambda$, temos:

$$
\forall x \in B, \exists z_{x} \in L(P ; \alpha) \text { tal que }\left\|x-z_{x}\right\|<\lambda(x)+\frac{\epsilon}{2} .
$$

Seja $\tilde{B}=B(0, R)$, onde $R=3 r+\lambda(0)+1$. Então, $z_{x} \in \tilde{B}$, pois:

$$
\left\|z_{x}\right\|<\|x\|+\lambda(x)+\frac{\epsilon}{2}<r+\lambda(x)+1 \leq r+2 r+\lambda(0)+1=R .
$$

Seja $\alpha_{n}(x)=P_{n}\left(z_{x}\right)$. Como $\alpha \neq 0$ e $\left|\alpha_{n}(x)-\alpha\right|=\left|P_{n}\left(z_{x}\right)-P\left(z_{x}\right)\right|$, a convergência uniforme de $\left(P_{n}\right)_{n \in \mathbb{N}}$ a $P$ em $\tilde{B}$ nos garante que

$$
\lim _{n \rightarrow \infty} \frac{\alpha}{\alpha_{n}(x)}=1
$$


uniformemente em $B$ - podemos assumir que $\alpha_{n}(x)$ nunca se anula. Se definirmos $z_{n}(x)=\left(\frac{\alpha}{\alpha_{n}(x)}\right)^{\frac{1}{k}} z_{x}$, temos:

$$
\left\|z_{n}(x)-z_{x}\right\|=\left|1-\left(\frac{\alpha}{\alpha_{n}(x)}\right)^{\frac{1}{k}}\left\|z_{x}\right\|\right|<\frac{\epsilon}{2}, \forall n>n_{0}(\epsilon) .
$$

Usando $(\star) e(\star \star)$, obtemos $\lambda_{n}(x)-\lambda(x)<\epsilon$, pois:

$$
\begin{gathered}
\lambda_{n}(x)-\lambda(x)<\lambda_{n}(x)-\left\|x-z_{x}\right\|+\frac{\epsilon}{2} \leq\left|\lambda_{n}(x)-\left\|x-z_{n}(x)\right\|\right|+\left\|z_{n}(x)-z_{x}\right\|+\frac{\epsilon}{2} \leq \\
\left\|z_{n}(x)-z_{x}\right\|+\frac{\epsilon}{2}<\epsilon, \forall n>n_{0}(\epsilon), \forall x \in B .
\end{gathered}
$$

Pode-se verificar a outra desigualdade de modo similar: definamos $z_{n, x} \in L\left(P_{n} ; \alpha\right)$ tal que $\left\|x-z_{n, x}\right\|<\lambda_{n}(x)+\frac{\epsilon}{2}$. Como $\lim _{n \rightarrow \infty} \lambda_{n}(0)=\lambda(0)$, podemos assumir que $z_{n, x} \in \tilde{B}$.

Se denotarmos $\alpha_{n}(x)=P\left(z_{n, x}\right), \tilde{z}_{n, x}=\left(\frac{\alpha}{\alpha_{n}(x)}\right)^{\frac{1}{k}} z_{n, x}$ e escolhermos $\eta>0$ de forma que:

$$
\left|\left(\frac{t}{t_{n}}\right)^{\frac{1}{k}}-1\right|<\frac{\epsilon}{2 R}, \text { sempre que }\left|t-t_{n}\right|<\eta,
$$

obtemos: $\exists n_{0}(\epsilon) \in \mathbb{N}$ tal que $\left|\alpha-\alpha_{n}(x)\right|=\left|P_{n}\left(z_{n, x}\right)-P\left(z_{n, x}\right)\right|<\eta, \forall n>n_{0}(\epsilon)$ (pela convergência uniforme de $\left(P_{n}\right)_{n \in \mathbb{N}}$ a $P$ em $\left.\tilde{B}\right)$ e, consequentemente:

$$
\begin{gathered}
\lambda(x)-\lambda_{n}(x)<\lambda(x)-\left\|x-z_{n, x}\right\|+\frac{\epsilon}{2} \leq \lambda(x)-\left\|\tilde{z}_{n, x}-x\right\|+\left\|\tilde{z}_{n, x}-z_{n, x}\right\|+\frac{\epsilon}{2} \leq\left\|\tilde{z}_{n, x}-z_{n, x}\right\|+\frac{\epsilon}{2}= \\
=\left|\left(\frac{\alpha}{\alpha_{n}(x)}\right)^{\frac{1}{k}}-1\right|\left\|z_{n, x}\right\|+\frac{\epsilon}{2}<\epsilon, \forall n>n_{0}(\epsilon), \forall x \in B .
\end{gathered}
$$

Concluímos, portanto, que $\lim _{n \rightarrow \infty} \lambda_{n}(x)=\lambda(x)$ uniformemente em $B$.

A união dos lemas 5.48 e 5.49 e as observações sobre o caso $P \equiv 0$ nos garantem o resultado procurado:

Teorema 5.50. Se $\left(P_{n}\right)_{n \in \mathbb{N}} \subset \mathcal{P}\left({ }^{k} E\right)$ e $P \in \mathcal{P}\left({ }^{k} E\right)$, então $\left(P_{n}\right)_{n \in \mathbb{N}}$ converge a $P$ uniformemente em conjuntos limitados se, e somente se, $L(P ; \alpha)=\mathrm{r}-\lim _{n \in \mathbb{N}} L\left(P_{n} ; \alpha\right), \forall \alpha \neq 0$.

O último passo é estudar o caso $\alpha=0$. Iniciemos com o caso complexo.

Proposição 5.51. Sejam E um espaço de Banach complexo, $\left(P_{n}\right)_{n \in \mathbb{N}} \subset \mathcal{P}\left({ }^{k} E\right) \backslash\{0\}$ e $P \in$ $\mathcal{P}\left({ }^{k} E\right) \backslash\{0\}$. Suponhamos que $\left(P_{n}\right)_{n \in \mathbb{N}}$ converge a $P$ uniformemente em subconjuntos limitados de E. Então $L(P ; 0)=\mathrm{r}-\lim _{n \in \mathbb{N}} L\left(P_{n} ; 0\right)$. 
Demonstração. Seja $B$ a bola unitária de $E$. Vamos mostrar que dados $r, \epsilon>0$ existe $n_{0} \in \mathbb{N}$ tal que $L\left(P_{n} ; 0\right) \cap r B \subseteq L(P ; 0)+\epsilon B, \forall n>n_{0}$. Se isso não ocorre, existem $r, \epsilon>0$ e uma sequiência $\left(x_{n}\right)_{n \in \mathbb{N}} \subseteq r B$ tais que $P_{n}\left(x_{n}\right)=0, \operatorname{mas} B\left(x_{n}, \epsilon\right) \cap L(P ; 0)=\emptyset$.

A convergência uniforme de $\left(P_{n}\right)_{n \in \mathbb{N}}$ a $P$ em $r B$ garante que $\lim _{n \rightarrow \infty} P\left(x_{n}\right)=0$. Escolhamos $z_{0} \in B$ tal que $P\left(z_{0}\right) \neq 0$, e definamos a função $\phi_{n}: D(0, \epsilon) \longrightarrow \mathbb{C}$ por $\phi_{n}(w)=P\left(x_{n}+w z_{0}\right)$. Então:

$$
\phi_{n}(w)=P\left(x_{n}\right)+\left(\begin{array}{c}
k \\
1
\end{array}\right) A\left(x_{n}, \cdots, x_{n}, z_{0}\right) w+\cdots++\left(\begin{array}{c}
k \\
k-1
\end{array}\right) A\left(x_{n}, z_{0}, \cdots, z_{0}\right) w^{k-1}+P\left(z_{0}\right) w^{k},
$$

onde $A$ denota a forma $k$-linear associada a $P$. Como $P\left(x_{n}\right) \rightarrow 0$, podemos afirmar que existe uma subsequiência de $\left(\phi_{n}\right)_{n \in \mathbb{N}}$ que converge a um polinômio $\phi$ tal que $\phi^{(k)}(0)=k ! P\left(z_{0}\right) \neq 0$. Mas:

$$
\phi(0)=\lim _{n \rightarrow \infty} \phi_{n}(0)=\lim _{n \rightarrow \infty} P\left(x_{n}\right)=0
$$

e isso, associado ao fato de que $\phi_{n}$ não se amula em $D(0, \epsilon)$, nos garante, a partir do corolário 5.21, que $\phi \equiv 0$, absurdo.

Vamos, agora, mostrar a outra inclusão: dados $r, \epsilon>0 \exists n_{0} \in \mathbb{N}$ tal que $\forall n>n_{0}, L(P ; 0) \cap r B \subseteq$ $L\left(P_{n} ; 0\right)+\epsilon B$. Procedemos de forma análoga: se isso não ocorre, existem $r, \epsilon>0$ e uma sequiència $\left(x_{n}\right)_{n \in \mathbb{N}} \subseteq r B$ tais que $P\left(x_{n}\right)=0$, mas $B\left(x_{n}, \epsilon\right) \cap L\left(P_{n} ; 0\right)=\emptyset$.

Seja $z_{0} \in B$ tal que $P\left(z_{0}\right) \neq 0$, e definamos $\phi_{n}, \tilde{\phi}_{n}: D(0, \epsilon) \longrightarrow \mathbb{C}$ por:

$$
\phi_{n}(w)=P\left(x_{n}+w z_{0}\right) \quad \text { e } \quad \tilde{\phi}_{n}(w)=P_{n}\left(x_{n}+w z_{0}\right)
$$

Note que $\tilde{\phi_{n}}$ não se anula. Escolhamos uma subseqüência de $\left(\phi_{n}\right)_{n \in \mathbb{N}}$ convergente a um polinômio $\phi$ tal que $\phi(0)=0$. É fácil ver que a subseqüência correspondente de $\left(\tilde{\phi_{n}}\right)_{n \in \mathbb{N}}$ também converge a $\phi$. Novamente, obtemos uma contradição pelo corolário 5.21 .

O exemplo 5.45 mostra que, no caso real, a convergência uniforme de $\left(P_{n}\right)_{n \in \mathbb{N}}$ em conjuntos limitados não implica a r-convergência de (ou mesmo a W-convergência) de $L\left(P_{n} ; 0\right)_{n \in \mathbb{N}}$ a $L(P ; 0)$.

$O$ exemplo seguinte mostra que, mesmo assumindo a W-convergência, a r-convergência dos conjuntos de nível 0 não segue da convergência uniforme de $\left(P_{n}\right)_{n \in \mathbb{N}}$ em conjuntos limitados, no caso real.

Exemplo 5.52. Seja $E=c_{0}(\mathbb{R})$, e tome os polinômios:

$$
P_{n}(x)=\sum_{k=1}^{n} \frac{1}{k^{2}} x_{k}^{2} \quad \text { e } \quad P(x)=\sum_{k=1}^{\infty} \frac{1}{k^{2}} x_{k}^{2}
$$

É fácil ver que $\left(P_{n}\right)_{n \in \mathbb{N}}$ converge uniformemente a $P$. Além disso, temos $L(P ; 0)=\{0\}$ e $L\left(P_{n} ; 0\right)=\left\{x \in c_{0}(\mathbb{R}): x_{1}=x_{2}=\cdots=x_{n}=0\right\}$. 
Temos que $\lim _{n \rightarrow \infty} \lambda_{n}(x)=\lambda(x), \forall x \in E$, já que $\lambda(x)=\|x\|$ e $\lambda_{n}(x)=\|x\|, \forall n>n_{0}$, onde $n_{0} \in \mathbb{N}$ é tal que $\left|x_{n}\right|<\frac{\|x\|}{2}, \forall n>n_{0}$. No entanto, se $B=B(0,1), L\left(P_{n}, 0\right) \cap 2 B$ nunca está incluso em $L(P ; 0)+\frac{1}{2} B$, pois $e_{n+1} \in L\left(P_{n}, 0\right) \cap 2 B$, mas $e_{n+1} \notin \frac{1}{2} B$, para todo $n \in \mathbb{N}$.

Observe que:

$$
d P(x)=2 \sum_{k=1}^{\infty} \frac{1}{k^{2}} x_{k} e_{k}^{\star}
$$

e, portanto:

$$
\|d P(x)\|_{1}=2 \sum_{k=1}^{\infty} \frac{1}{k^{2}}\left|x_{k}\right|
$$

Logo, $\inf _{\|x\|=1}\|d P(x)\|_{1}=0$.

Esse fato sugere o seguinte resultado:

Proposição 5.53. Sejam $E$ um espaço de Banach real, $\left(P_{n}\right)_{n \in \mathbb{N}} \subset \mathcal{P}\left({ }^{k} E\right)$ e $P \in \mathcal{P}\left({ }^{k} E\right)$ tais que:

$$
\inf _{\|x\|=1}\|d P(x)\|_{1}>0
$$

Se a seqüência $\left(P_{n}\right)_{n \in \mathbb{N}}$ converge a $P$ uniformemente em subconjuntos limitados, então $L(P ; 0)=\mathrm{r}-\lim _{n \in \mathbb{N}} L\left(P_{n} ; 0\right)$.

Demonstração. Seja $B$ a bola unitária de $E$. Primeiramente, vamos mostrar que dados $r, \epsilon>0$ existe $n_{0} \in \mathbb{N}$ tal que $L\left(P_{n} ; 0\right) \cap r B \subseteq L(P ; 0)+\epsilon B, \forall n>n_{0}$.

Se isso não ocorre, existem $r, \epsilon>0$ e uma seqüência $\left(x_{n}\right)_{n \in \mathbb{N}} \subseteq r B$ tais que $P_{n}\left(x_{n}\right)=0$, mas $B\left(x_{n}, \epsilon\right) \cap L(P ; 0)=\emptyset$. A convergência uniforme de $\left(P_{n}\right)_{n \in \mathbb{N}}$ em $r B$ garante que $\lim _{n \rightarrow \infty} P\left(x_{n}\right)=0$.

Como $x_{n} \in r B \backslash \epsilon B, \forall n \in \mathbb{N}$, existe uma constante $c>0$ tal que $\left\|d P\left(x_{n}\right)\right\|>c, \forall n \in \mathbb{N}$. Logo, podemos escolher $y_{n} \in B(0,1)$ tal que $\left|d P\left(x_{n}\right)\left(y_{n}\right)\right|>c, \forall n \in \mathbb{N}$.

Definamos $\phi_{n}:(-\epsilon, \epsilon) \longrightarrow \mathbb{R}$ por $\phi_{n}(r)=P\left(x_{n}+r y_{n}\right)$. Como $P\left(x_{n}\right) \rightarrow 0$, podemos afirmar que uma subseqüência de $\left(\phi_{n}\right)_{n \in \mathbb{N}}$ converge a um polinômio $\phi$ tal que:

$$
\phi(0)=\lim _{n \rightarrow \infty} \phi_{n}(0)=\lim _{n \rightarrow \infty} P\left(x_{n}\right)=0 .
$$

$\mathrm{O}$ fato de que $\phi_{n}$ não se anula nos dá, pelo lema 5.26 , que $\phi^{\prime}(0)=0 \mathrm{ou}$, equivalentemente, $\lim _{n \rightarrow \infty} d P\left(x_{n}\right)\left(y_{n}\right)=0$, absurdo.

Mostremos, agora, a outra inclusão: dados $r, \epsilon>0$ existe $n_{0} \in \mathbb{N}$ tal que $\forall n>n_{0}, L(P ; 0) \cap r B \subseteq$ $L\left(P_{n} ; 0\right)+\epsilon B$.

Se isso não ocorre, existem $r, \epsilon>0$ e uma seqüência $\left(x_{n}\right)_{n \in \mathbb{N}} \subseteq r B$ tais que $P\left(x_{n}\right)=0$, mas $B\left(x_{n}, \epsilon\right) \cap L\left(P_{n} ; 0\right)=\emptyset$. Considerando $\left(y_{n}\right)_{n \in \mathbb{N}}$ como na primeira parte da prova, definimos 
$\phi, \phi_{n}:(-\epsilon, \epsilon) \longrightarrow \mathbb{R}$ por:

$$
\phi(r)=\lim _{n \rightarrow \infty} P\left(x_{n}+r y_{n}\right) \quad \text { e } \quad \phi_{n}(r)=P_{n}\left(x_{n}+r y_{n}\right)
$$

passando a uma subseqüencia, se necessário. Observe que $\phi_{n}$ nunca se anula, e $\left(\phi_{n}\right)_{n \in \mathbb{N}}$ converge a $\phi$, pela convergência uniforme de $\left(P_{n}\right)_{n \in \mathbb{N}}$ a $P$ em $(r+\epsilon) B$. Mas $\phi(0)=0$ e então, pelo lema 5.26, $\phi^{\prime}(0)=0$, donde $\lim _{n \rightarrow \infty} d P\left(x_{n}\right)\left(y_{n}\right)=0$, absurdo.

Para finalizar, observamos aqui que não faz sentido estudar a convergência, no sentido de Mosco, de conjuntos de nível de polinômios homogêneos. Ao contrário do que ocorre no caso dos funcionais lineares contínuos, os conjuntos de nível dos polinômios não são convexos. Isso explica a ausência desse tipo de convergência de conjuntos no artigo [8]. 


\section{Bibliografia}

[1] Beer, G. Metric spaces with nice closed balls and distance functions for closed sets. Bull. Austral. Math. Soc. 35 (1987), 81-96.

[2] Beer, G. On Mosco convergence of convex sets. Bull. Austral. Math. Soc. 38 (1988), 239-253.

[3] Beer, G. Convergence of continous linear functionals and their level sets. Arch. Math. 52 (1989), 482-491.

[4] Brown, A. L. A rotund reflexive space having a subspace of codimension two with a discontinous metric projection. Michigan Math. J. 21 (1974), 145-151.

[5] Coelho, F. U., Lourenço, M. L. Um Curso de Álgebra Linear. EDUSP, São Paulo, 2001.

[6] Conway, J. B. Functions of One Complex Variable I. Springer-Verlag, New York, 1978.

[7] Diestel, J. Sequences and Series in Banach Spaces. Springer-Verlag, New York, 1984.

[8] Ferrera, J. Convergence of polynomial level sets. Transactions of the Amer. Math. Soc. vol. 350 , Number 12 (1998), 4757-4773.

[9] Kreyszig, E. Introductory Functional Analysis with Applications. John Wiley and Sons, 1978.

[10] Kuratowski, K. Topology - Vol. 1. Academic Press, New York and London, 1966.

[11] Lages, E. L. Espaços Métricos. LTC, Rio de Janeiro, 1993.

[12] Megginson, R. E. An Introduction to Banach Space Theory. Springer-Verlag, New York, 1998.

[13] Mosco, U. Convergence of convex sets and solutions of variational inequalities. Adv. in Math. 3 (1969), 510-585.

[14] Mujica, J. Notas de Espaços Vetoriais Topológicos. Notas de Aula, IMECC - UNICAMP, 2004. 
[15] Pellegrini, L. Um Teorema de Hahn-Banach para Polinômios Homogêneos. Dissertação de Mestrado, IME - USP, 2001.

[16] Schur, I. Über lineare Transformationen in der Theorie der unendlichen Reihen. Journal Reine Angew. Math. 1551 (1921), 79-111. 